\title{
An Investigation of the Irrotational Near Field of an Excited High Speed Jet
}

\section{THESIS}

Presented in Partial Fulfillment of the Requirements for the Degree Master of Science in the Graduate School of The Ohio State University

\author{
By \\ Hind Alkandry, B.S. \\ Graduate Program in Mechanical Engineering
}

The Ohio State University

2013

Master's Examination Committee:

Dr. Datta Gaitonde

Dr. Mo Samimy, Advisor 
Copyright by

Hind Alkandry

2013 


\begin{abstract}
The near-field pressure of a Mach 0.9 jet with Reynolds number of $6.2 \times 10^{5}$ has been investigated to examine the response of the jet to low-frequency forcing with localized arc filament plasma actuators (LAFPAs). The well-defined actuation phase allows for the phase averaging of the near-field pressure in order to separate the relevant features of the response from the background turbulence. The resulting phase-averaged signal illustrates a compact wave with a positive pressure excursion preceding a negative one. This is indicative of a large-scale structure (a vortex ring) that is generated by each impulsively seeded perturbation. The signature of these large-scale structures is shown to rapidly decay with radial distance from the jet. Simultaneous measurements of acoustic far field were also performed along with the near field, allowing the relationship between the two fields to be examined. Although the region near the jet shear-layer is largely hydrodynamic in nature, there is still significant correlation between the near-field pressure and the far-field acoustic around $30^{\circ}$ polar angle, particularly as the jet evolves downstream past the potential core. This region is shown to shift upstream when the jet is forced.
\end{abstract}




\section{Dedication}

This document is dedicated to my family. 


\section{Acknowledgments}

I would like to express my gratitude to my family, advisor, colleagues, and friends that have helped me throughout my studies at The Ohio State University.

First and foremost I would like to thank my parents and brother, to whom this work is dedicated, for always being there for me and supporting me throughout my life. I would like to acknowledge my advisor, Dr. Mo Samimy, for his guidance and especially for giving me this excellent research opportunity. I would also like to thank Dr. Gaintonde for kindly agreeing to serve as my second committee member.

I would like to thank all of the students of the GDTL for their continued support and assistance throughout my years at the lab. I must particularly thank Dr. Aniruddha Sinha, Michael Crawley, and Dr. Martin Kearney-Fischer for the assistance and advice each provided for much of the work presented in this thesis. It has been a pleasure working with all of you and I could not have done this work without you.

Lastly I would like to thank the Air Force Office of Scientific Research with John Schmisseur for providing the funding that has made this research possible. 


\section{Vita}

February 12, 1988

Born - Rabat, Morocco

2009

Undergraduate Student Researcher

Department of Mechanical and Aerospace Engineering Syracuse University

2010

B.S. Aerospace Engineering

Syracuse University

2010-2011

Graduate Teaching Assistant

Department of Mechanical and Aerospace Engineering

The Ohio State University

2011-present

Graduate Research Associate Gas Dynamics and Turbulence Laboratory

Department of Mechanical and Aerospace Engineering

The Ohio State University

\section{Publications}

Alkandry, H., Crawley, M., Sinha, A., Kearney-Fischer, M., and Samimy, M., 2013, "An Investigation of the Irrotational Near Field of an Excited High-Speed Jet," 51st AIAA Aerospace Sciences Meeting, AIAA.

Sinha, A., Alkandry, H., Kearney-Fischer, M., Samimy, M., and Colonius, T., 2012, "The Impulse Response of a High-Speed Jet Forced with Localized Arc Filament Plasma Actuators," Phys. Fluids, 24, pp. 24-44.

Sinha, A., Alkandry, H., Kearney-Fischer, M., and Samimy, M., 2012, "The Impulse Response of a High-Speed Jet Forced with Localized Arc Filament Plasma Actuators," 18th AIAA/CEAS Aeroacoustics Conference, AIAA.

\section{Fields of Study}

Major Field: Mechanical Engineering

Studies In: Fluid Dynamics, Flow Control, High-Speed Jets, Aeroacoustics 


\section{Table of Contents}

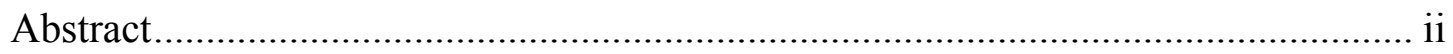

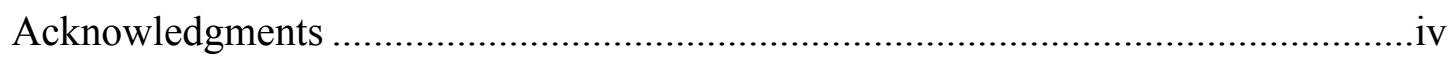

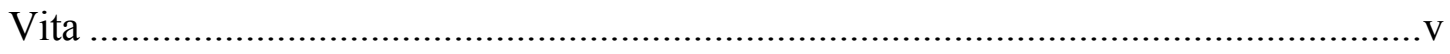

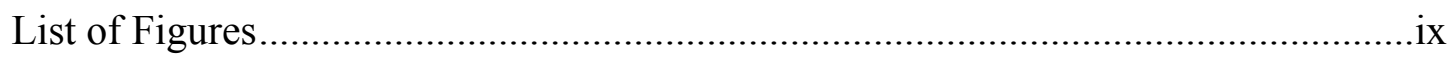

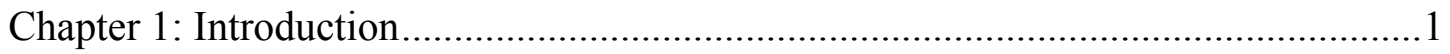

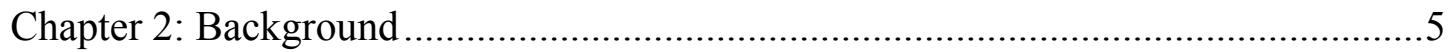

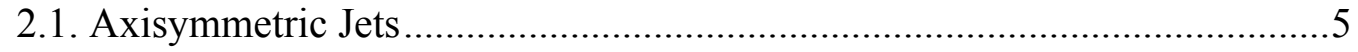

2.1.1. Structures of High-Speed Jets ................................................ 6

2.1.2. Jet Noise ............................................................................... 7

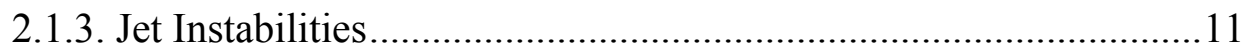

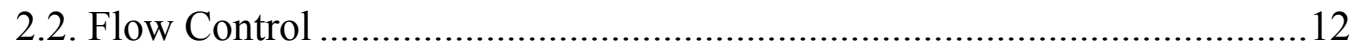

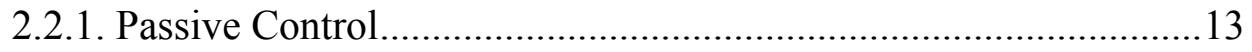

2.2.2. Active Control .................................................................... 13

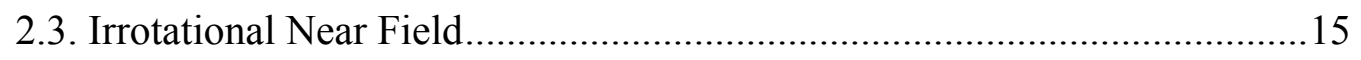

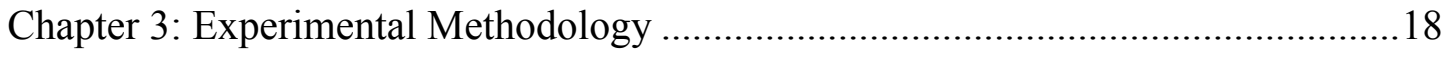

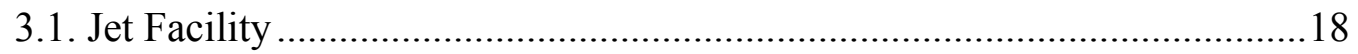

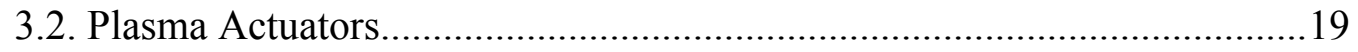


3.3. Data Acquisition

3.3.1. Near and Far Field...................................................................21

3.3.2. Actuation Phase ..........................................................................24

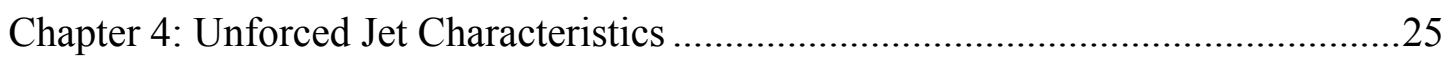

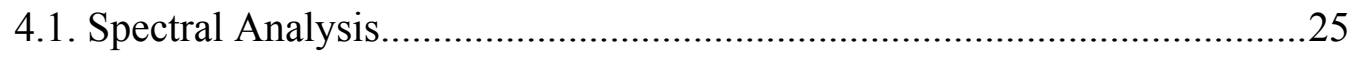

4.1.1. Far-Field Spectra ................................................................25

4.1.2. Near-Field Power Density Spectra............................................26

4.1.3. Mean-Square of the Near-Field Pressure Fluctuations .................29

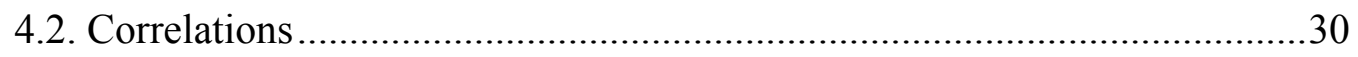

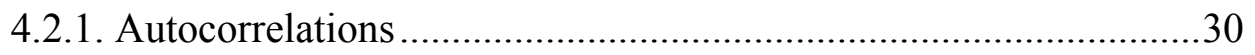

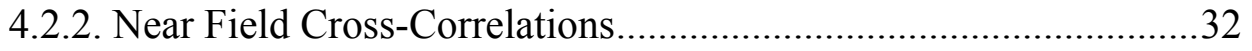

4.2.3. Near Field to Far Field Cross-Correlations ..................................34

Chapter 5: Effects of Forcing: Statistical …..................................................... 40

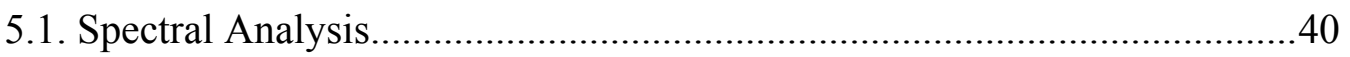

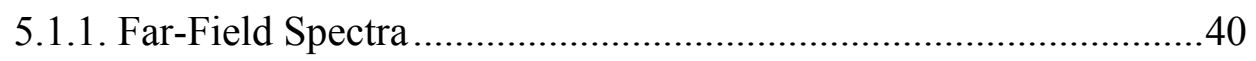

5.1.2. Mean-Square of the Near-Field Pressure Fluctuations .................41

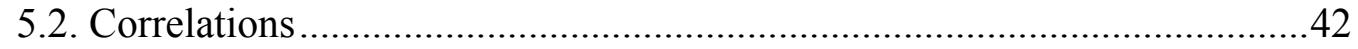

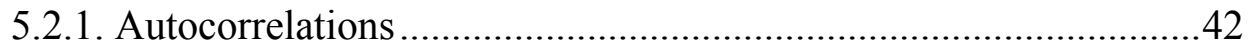

5.2.2. Near Field Cross-Correlations..................................................44 
Chapter 6: Effects of Forcing: Phase-Averaging ..................................................49

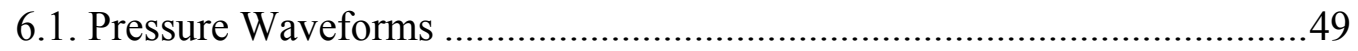

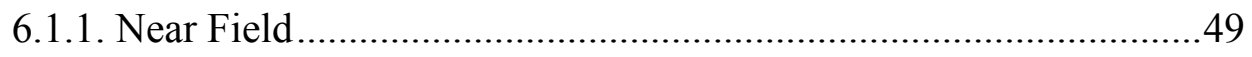

6.1.2. Far Field ..........................................................................5

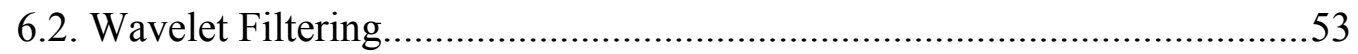

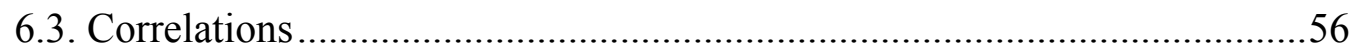

6.3.1. Autocorrelations ...................................................................56

6.3.2. Near Field Cross-Correlations.................................................59

6.3.3. Near Field to Far Field Cross-Correlations .................................61

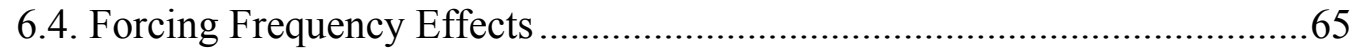

6.4.1. Phase-Averaged Waveforms .................................................65

6.4.2. Autocorrelations ................................................................6 66

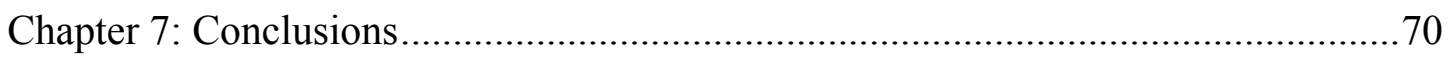

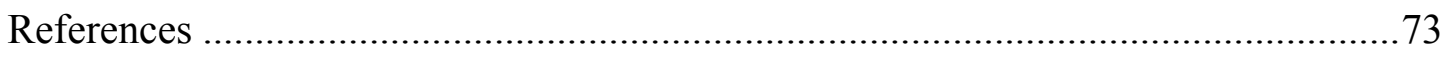




\section{List of Figures}

Figure 1. Schematic of an axisymmetric jet.

Figure 2. Variation in the far-field spectra with different observation angles, $\theta$, in degrees for a Mach 0.9 jet. 9

Figure 3. Diagram of the flow regimes of a round jet in the radial direction. The solid line depicts the time-averaged pressure fluctuation of the axisymmetric mode and the dotted line denotes the corresponding eigenfunction calculated from linear stability analysis [26] .16

Figure 4. Schematic of anechoic chamber and jet. ..................................................18

Figure 5. (a) LAFPAs housed in nozzle extension, (b) Schematic of high-voltage circuitry for one LAFPA channel....... 20

Figure 6. (a) Linear array for pressure measurements, (b) Microphone array grid coordinates for irrotational pressure field measurements. .22

Figure 7. Far-field spectra of a Mach 0.9 axisymmetric jet. .......................................26

Figure 8. Power spectral density for unforced jet: (a) PSD for first array position at various axial locations, (b) PSD at $x / D=5$ for three radial locations. .28

Figure 9. Mean-square of the near-field pressure fluctuations for unforced jet..........30

Figure 10. Normalized autocorrelations of the unforced jet at the same axial position and various radial locations: (a) $x / D=6$, (b) $x / D=9$. 32 
Figure 11. Normalized cross-correlations of the near-field pressure fluctuations for the unforced jet with the reference microphone at $x / D=9$ : (a) First array location, (b)

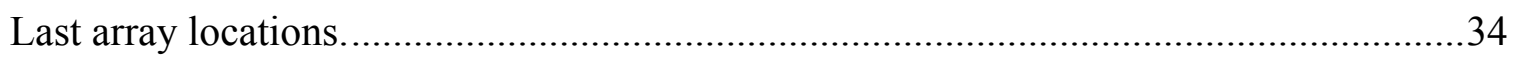

Figure 12. Normalized cross-correlation of the near-field pressure fluctuations for the unforced jet with the $30^{\circ}$ far-field microphone: (a) First array location, (b) Last array location. 36

Figure 13. Normalized cross-correlation of the near-field pressure fluctuations for the unforced jet with the $30^{\circ}$ far-field microphone for the first array location. 37

Figure 14. Maximum normalized cross-correlations of the near-field pressure fluctuations for the unforced jet: (a) With $30^{\circ}$ far-field microphone, (b) With $60^{\circ}$ far-field microphone. 39

Figure 15. Far-field SPL at $\theta=30^{\circ}$ for the Mach 0.9 jet forced with $\mathrm{m}=0$ at $S t_{D F}=0.02$

Figure 16. Mean-square of the near-field pressure fluctuations for the jet force at $S t_{D F}=0.02$

Figure 17. Normalized autocorrelations of the forced jet at the same axial position and various radial locations: (a) $x / D=6$, (b) $x / D=9$.

Figure 18. Normalized autocorrelation of the forced jet for $x / D=9$ at the first array location.

Figure 19. Normalized cross-correlations of the near-field pressure fluctuations for the forced jet with the reference microphone at $x / D=9$ : (a) First array location, (b) Last array location. .45 
Figure 20. Normalized cross-correlation of the near-field pressure fluctuations for the forced jet with the $30^{\circ}$ far-field microphone: (a) First array location, (b) Last array location.

Figure 21. Maximum normalized cross-correlations of the near-field pressure fluctuations for the forced jet with the $30^{\circ}$ far-field microphone. 48

Figure 22. Phase-averaged waveform for $S t_{D F}=0.02$ : (a) $\mathrm{x} / \mathrm{D}=2, \mathrm{r} / \mathrm{D}=1.28$, (b) First array location for all microphones.

Figure 23. Phase-averaged waveform for $S t_{D F}=0.02$ : (a) $\mathrm{x} / \mathrm{D}=2$ and 9 for the last array locations, (b) Last array location for all microphones .52

Figure 24. Phase-averaged waveform for the $30^{\circ}$ far-field microphone. .53

Figure 25. Phase-averaged waveforms for original and filtered waveforms: (a) $x / D=2.0, r / D=1.28$, (b) $x / D=2, r / D=3.68$. .55

Figure 26. $4^{\text {th }}$ Order Paul wavelet sample. .56

Figure 27. Normalized autocorrelations of the phase-averaged waveforms at the same axial position and various radial locations: (a) $x / D=6$, (b) $x / D=9$. .58

Figure 28. Normalized autocorrelations of the phase-averaged waveforms at $x / D=9$ : (a) First array location, (b) Last array location.

Figure 29. Normalized cross-correlations of the phase-averaged waveforms with the reference microphone at $x / D=9$ : (a) First array location, (b) Last array location.

Figure 30. Normalized cross-correlations of the phase-averaged waveforms with the $30^{\circ}$ far-field microphone: (a) First array location, (b) Last array location. .63 
Figure 31. Maximum normalized cross-correlations of the phase-averaged waveforms with the $30^{\circ}$ far-field microphone.

Figure 32. Phase-averaged waveform for $S t_{D F}=0.15$ : (a) First array location, (b) Last array location. .66

Figure 33. Normalized autocorrelations of the forced jet at $S t_{D F}=0.15$ at $\mathrm{x} / \mathrm{D}=9$ for various radial locations: (a) Instantaneous near-field pressure, (b) Phase-averaged waveform. .68

Figure 34. Normalized autocorrelations at $\mathrm{x} / \mathrm{D}=9$ : (a) First array location, (b) Last array location. .69 


\section{Chapter 1: Introduction}

Jet noise has been an issue of enormous environmental, technological, and financial impact to both the civilian and military aircraft industry. The composition of jet noise and the relative strength of the noise sources are highly dependent on the jet temperature and Mach number. It is well established that there exists flow structures ranging in size from dissipation-scale to the order of the exit dimension of the jet nozzle. It is generally accepted that jet noise is produced by the interaction and disintegration of these structures [1]. In subsonic jets, the interaction and disintegration of flow structures produce what is known as mixing noise. A supersonic jet could also include screech tones, broadband shock associated noise, and Mach wave radiation. Various attempts have been made to manipulate the flow structures found in the shear layer (utilizing both active and passive control strategies) to reduce the resulting noise with varying degrees of success. The purpose of this thesis is to use active control on a high-speed subsonic jet to better understand the relationship between the irrotational pressure field (and thus the jet flow dynamics) and the radiated noise to the far field. Active control facilitates the study of the noise production mechanisms by isolating particular flow structures for study. Furthermore, forcing the jet provides a well-defined phase relationship allowing for phase-locked data acquisition for a better understanding of the jet dynamics.

Jet flow control is a well-researched topic with applications including, but not limited to, noise mitigation and mixing enhancement. Flow control methods can be divided into 
two basic categories: passive (geometrical modifications of the nozzle such as chevrons, lobed nozzles, etc.) [2-6] and active (modifiers which can be turned off to eliminate performance penalties when unneeded such as fluidic actuators, plasma actuators, etc.)[713]. Active flow control methods are desirable, but achieving control authority in highspeed and high Reynolds number flows is challenging.

Jets have three different instabilities, which have been well researched at low speeds and low Reynolds numbers [7-14]: the jet initial shear layer instability referred to as the Kelvin-Helmholtz instability, the jet column or jet preferred mode instability and, in the case of an axisymmetric jet, the azimuthal instability. More information on these instabilities and how they are relevant to the control of jets can be found in Section 2.1.3 and previous works $[15,16]$.

An important class of active flow control involves the excitation of flow instabilities. One or more of the flow instabilities mentioned above might be manipulated in order to actively control jets. As the speed and the Reynolds number of the jet increase, so do the background noise, the instability frequencies, and the flow momentum. The relatively few previous attempts at active flow control of highly energetic jets have included the use of acoustic drivers, fluidic actuators, and piezoelectric actuators to control high subsonic jets around their preferred mode [17-19]. Unfortunately, acoustic drivers lose control authority in high Reynolds numbers, while fluidic and piezoelectric actuators are limited to narrow-band and lower frequencies. In order to operate in this environment, actuators must provide excitation signals with much higher amplitudes and frequencies. 
Localized arc filament plasma actuators (LAFPAs) have been developed and implemented for active control of jets at The Ohio State University over the past several years. The Gas Dynamics and Turbulence Laboratory (GDTL) at The Ohio State University has used these actuators for noise mitigation and flow control in a variety of flows and significant control authority on these flows has been demonstrated $[15,16,20$ 24]. LAFPAs have also demonstrated the effect of organizing the jet shear layer allowing for the creation of large-scale structures in the flow with periodicity closely matching the excitation.

The irrotational near-field pressure has also been examined (both by GDTL and by other researchers in literature) [25-28], but not as extensively as the jet flowfield and acoustic far field mentioned above. The near-field pressure consists of both hydrodynamic and acoustic signals. The acoustic signal is the propagative part of the pressure signal while the hydrodynamic pressure is characterized by exponential decay of these fluctuations with increasing radial distance $[29,30]$. Furthermore, the hydrodynamic field contains the convective signature of the large-scale structures in the shear layer. The earlier work done by Sinha et al. [28] was an initial validation of our hypothesis that large-scale structures are generated with regularity by the impulsive forcing, so that even at very low frequencies of forcing we can discern them clearly in the phase-averaged near-field pressure. These pressure perturbations could be explained by linear parabolized stability equations. Structure interaction was shown to set in when the forcing frequency approaches the jet column mode frequency, but a quasi-linearity was observed in the interactions for frequencies somewhat beyond the jet column mode [28]. 
In this thesis, an extension of the above study is reported. Specifically, the region of the near field measurements is significantly enlarged. Moreover, the far field acoustics are acquired simultaneously. Finally, extensive correlation studies are conducted to investigate the details of noise propagation within the near field, and from the near field to the far field. The overall goal is to use near-field pressure measurements to further characterize the effect of LAFPA forcing on jets and to better understand the jet dynamics associated with the radiating noise. 


\section{Chapter 2: Background}

Jet noise is a problem that has plagued the use of jet engines since the start of commercial jet travel. The majority of noise during the take-off phase of a jet aircraft is due to the exhaust of jet engines, and thus has been a topic of research over the past sixty plus years. The goal in the present work is to relate the large-scale dynamics in a highspeed subsonic, axisymmetric jet to its far-field acoustics and to provide a better understanding of noise sources. This chapter provides a review of what has been done to study this relationship and jet noise in general. First, an overview of an axisymmetric jet is given including a discussion of the structures in a high-speed jet and the interaction of these structures. Second the jet instabilities are discussed before moving on to flow control. Lastly, the irrotational near field of a high-speed jet is examined.

\subsection{Axisymmetric Jets}

In this work an axisymmetric jet is investigated, so a brief description of an axisymmetric jet is provided. In the present experiment, the jet is exiting into a quiescent atmosphere. Immediately downstream of the nozzle exit, the jet-mixing layer is formed. This is the region where ambient fluid is entrained into the jet and mixing between the two occurs. For axisymmetric jets, the mixing layer grows as a circular ring of shrinking inner diameter. Furthermore, for high-Reynolds number flows the mixing layer of the jet is highly turbulent. The mixing layer comes together at the end of the potential core. The 
potential core of the jet is a region of irrotational fluid moving at relatively constant velocity (i.e. the jet exit velocity). Downstream of the potential core, the average centerline velocity of the jet starts to decrease, and after a transition region, the centerline velocity decreases at a rate of $1 / \mathrm{x}$ (where $\mathrm{x}$ is the distance along the jet centerline downstream of the nozzle-exit) [31]. A schematic of an axisymmetric jet is shown in Figure 1. The basic features of the jet may have been briefly described in the present paragraph, but as is usually the case, the details are far more elusive.

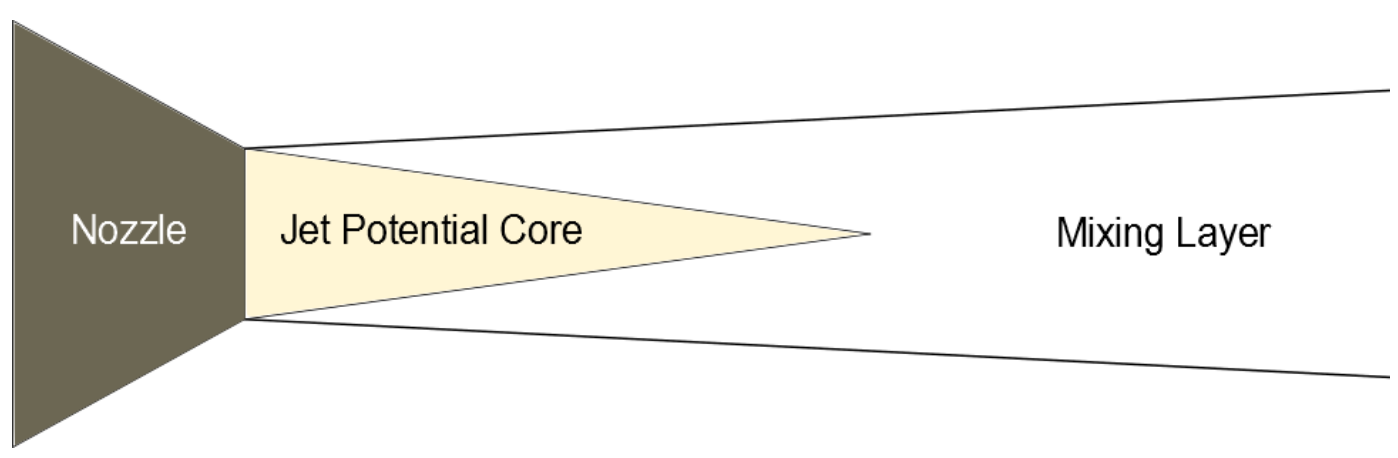

Figure 1. Schematic of an axisymmetric jet.

\subsubsection{Structures of High-Speed Jets}

In any turbulent flow, there are coherent structures that vary significantly in scale. For the $2.54 \mathrm{~cm}$ (one inch) diameter, Mach 0.9 jet of this study the Reynolds number based on the nozzle-exit diameter is $\sim 6.5 \times 10^{5}$, making the mixing layer highly turbulent. The structures found in a jet form immediately past the nozzle exit, and grow into large-scale structures as they convect to some distance beyond the end of the potential core. Downstream of the nozzle, the large turbulent structures draw energy from the flow and 
grow by entrainment, then interaction spanning the width of the jet past the end of the potential core. Past the potential core, the large-scale structures cascade to smaller scale structures, which dissipate energy by friction and are of a size determined by the Kolmogorov scale. The ratio of the Kolmogorov scale to the largest structures is on the order of $\operatorname{Re}_{D^{-}}^{-0.75}$ [32]. It is to be noted that the most turbulent area of a jet lies in the first two potential core lengths [33]. This region is also considered to be the most dynamically energetic.

Large-scale structures are believed to be the cause of the most intense component of jet noise. Before the 1970s, jet turbulence was thought to consist of numerous small eddies distributed throughout the jet. It was not until the works of Crow \& Champagne [7] and Brown \& Roshko [34] that the existence of large-scale coherent structures at high Reynolds number shear layers became known. The large structures form immediately past the nozzle exit due to Kelvin-Helmholtz instabilities. The inflection point in the initial velocity profile, needing only a small disturbance of proper frequency to destabilize the flow, leads to a rollup of the fluid into a coherent structure. This initially small structure, which is contained within the mixing layer and entraining fluid from the ambient, convects downstream, and will grow past the end of the potential core. Downstream of the end of the potential core, this structure will eventually decay into smaller scales.

\subsubsection{Jet Noise}

It is generally accepted that jet noise is produced by the interaction, as well as the disintegration of the structures in the flow. The noise from a high-speed jet has various 
components that are highly dependent on the jet temperature and Mach number. In subsonic jets or ideally expanded supersonic jets, the evolution, interaction, and disintegration of flow structures produce what is known as turbulent mixing noise. A supersonic jet could also include screech and broadband shock-associated noise, as well as Mach wave radiation.

There is universal agreement that jet-mixing noise is generated by the turbulence of the jet flow. With the introduction of large-scale structures due to the works of Crow and Champagne [7] and Brown and Roshko [34], new thinking about turbulence-generated noise with a focus on the generation of sound by large-scale structures prospered. In moderate to high Reynolds number jets, the large-scale structures radiate predominantly downstream towards shallow angles to the jet $[35,36]$. Similarly, the acoustic field at these shallow angles $\left(\theta \sim 30^{\circ}\right.$ with respect to the jet axis) is dominated by a moderately narrow distribution of frequencies $\left(S t_{D} \sim 0.1\right.$ to 0.5$)$ as has been well established by several researchers $[11,37,38]$. This can also be seen in Figure 2, which represents a Mach $0.9(D=0.0254 \mathrm{~m})$ jet acoustic spectra given for various observation angles, $\theta$. These spectra are from the same jet that is presented in this thesis. The sound spectrum at shallow angles $\left(30^{\circ}\right)$ has a broadband peak with a maximum at $S t_{D}=0.17$; on the other hand, at angles normal to the jet exit $\left(90^{\circ}\right)$ the spectrum is relatively uniform over most frequencies. 


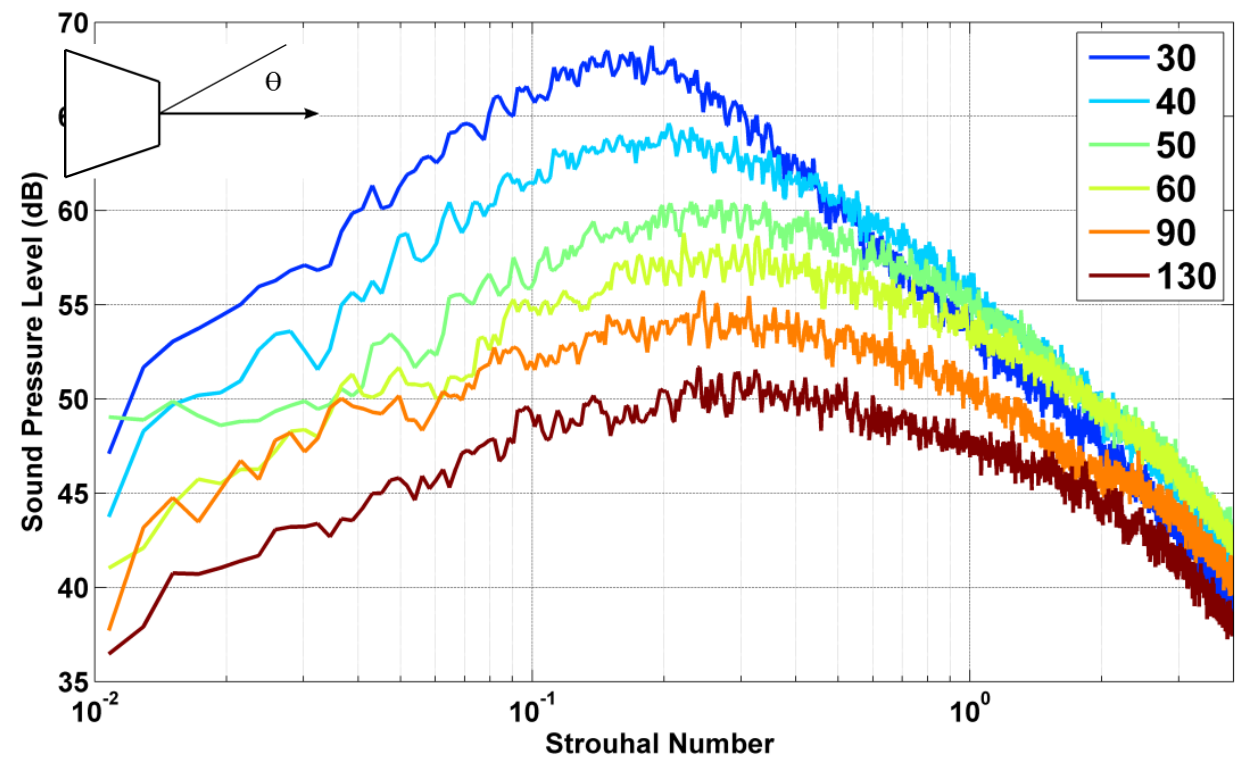

Figure 2. Variation in the far-field spectra with different observation angles, $\theta$, in degrees for a Mach 0.9 jet.

Some researchers have proposed to divide the turbulent mixing noise into two distinct components $[39,40]$. The first component is due to the large-scale structures and radiates preferentially downstream, while the other component is due to the small-scale structures and radiates to the sideline and upstream angles. Tam et al. [41] used 1900 spectra taken at the Jet Noise Laboratory of the NASA Langley Research Center to empirically create two seemingly universal spectra that were able to fit all the jet noise spectra. These similarity spectra were able to model the two noise components regardless of jet Mach number or jet temperature. These similarity spectra have since been found to accurately recreate spectra from axisymmetric subsonic jets $[42,43]$. Although it is generally accepted that this empirical model provides a reasonable representation of the shapes of 
the spectra in jets dominated by turbulent mixing noise, there is still debate about the implications of it within the jet noise research community.

Within moderate to high Reynolds number jets, the majority of the turbulent mixing noise radiates from a region that surrounds the end of the potential core [44]. Schaffar [45] found that the noise source region of a Mach 0.98 jet was between 5 and 10 jet diameters downstream of the nozzle exit. This was obtained using the correlation between velocity fluctuations inside the jet and the far-field acoustic pressure. This was also supported by looking at acoustic phase fronts [46] and microphone array data [47, 48].

An understanding of the large-scale structures is needed in order to understand how they generate noise. The seemingly easiest way to study both the dynamics of large-scale structures and the production of sound is to perform simultaneous point measurements in the flow and acoustic fields [49]. However, due to the inherent issues in making these simultaneous point measurements, much of the understanding of the generation of noise has come from analyzing the time average properties of the jet in the region of maximum noise generation. In this thesis, near-field pressure measurements, made outside of the jet shear layer, along with far field pressure measurements are used to gain a better understand of the dynamics of the large-scale structures and gain insight into noise propagation. 


\subsubsection{Jet Instabilities}

Jets have several instabilities that have been well researched in low-speed and low Reynolds number jets. These instabilities are: the shear layer instability (related to the initial shear layer that forms past the nozzle exit) and the jet column instability (due to the instability of the potential core). In the case of axisymmetric jet, the nature of the large structures is also defined based on the azimuthal instability.

The first flow instability is the initial shear layer instability, which is referred to as the Kelvin-Helmhotz instability. This instability leads to the formation of large-scale structures in the jet-mixing layer. These structures entrain the ambient fluid into the mixing layer from both sides and play a major role in the mixing of the fluids [20]. The naturally occurring frequency for this mode within unforced mixing layers was theoretically determined to be $S t_{\theta}=0.017$ [50], where the Strouhal number is based on the jet's initial momentum thickness, $\theta$ ( $S t_{\theta}=f \theta / U_{j}$, where $U_{j}$ is the jet exit velocity and $f$ is the frequency). However, many researchers have found that this number actually varies in experiments from 0.009 to 0.023 [11].

The second type of instability is that of the jet column, also referred to as the preferred mode of the jet. This instability is amplified further downstream near the end of the potential core, and its frequency is scaled with the nozzle exit diameter, $D$ $\left(S t_{D}=f D / U_{j}\right)$. The first measurement of the jet preferred mode was performed by Crow and Champagne [7] who found that forcing the jet at a frequency of $S t_{D}=0.3$ caused the largest amplification in the large-scale structures at the end of the potential core. The experiment was conducted by applying acoustic forcing to the jet over a wide range of 
frequencies. It was since determined experimentally that the maximum amplification of this instability varies over a range of $S t_{D}$ from 0.2 to 0.6 [11]. The optimum Strouhal number for this instability is heavily dependent upon the experimental facility due to the variations of the naturally occurring disturbances in the experiments, as well the measurement technique.

In addition to the initial shear layer and the jet column mode instabilities, axisymmetric jets are susceptible to azimuthal mode instability. This instability dictates how the large-scale structures will develop within the jet shear layer. For instance, the azimuthal instability of order $\mathrm{n}=0$ leads to the development of an axisymmetric largescale structure that is uniform over any jet cross-section. The growth of various modes of this instability depends on the ratio of the jet diameter to the initial shear layer momentum thickness $(D / \theta)$ and on the streamwise location. More information on these instabilities can be found in previous works [20, 51, 52].

\subsection{Flow Control}

The shear layer of a jet can be perturbed in many ways to control the production of large-scale structures for the purpose of noise reduction or mixing enhancement. Previous studies of jet flow control include both passive and active control techniques, both of which will be further discussed next. 


\subsubsection{Passive Control}

Passive control is accomplished by geometrical modifications to the nozzle. These modifications include for example triangular tabs or chevrons that protrude into the flow from the nozzle exit. The main mechanism using these passive techniques is streamwise vorticity generated by the geometrical modifications [3]. The streamwise vortices are produced by the spanwise pressure gradient created by the tabs. The generated vortices improve the flow mixing and can alter the noise generated by the jet. These techniques do not require any power, however, they incur performance penalties such as loss of thrust since they are always on even when they are not needed. Chevrons are similar to the tabs in terms of generated effects, but have significantly less area blockage and thus have less thrust losses [5]. For this reason, they are currently preferred in industry. Ideally, the nozzle would be able to utilize the effects of the tabs or chevrons only during the beginning and end of the flight, when noise radiation is most harmful, but disengage them when at cruise and there is less need for noise control. This would eliminate some of the thrust losses incurred by the modifications when they are actively influencing the flow despite little to no benefit.

\subsubsection{Active Control}

Another way of alternating the flow in a jet is by active control. Active control involves the addition of some energy, momentum, or mass to the flow. This control can be turned on or off as needed, limiting the performance penalties. Active flow control is further divided into two categories: open-loop and closed-loop (feedback) control. In open-loop flow control, the input is predetermined and the actuation is based on the 
operators command. In the closed-loop flow control, information obtained in real-time from sensors in the flow is used by a flow model which guides the actuation process. Active flow control can also be classified by two mechanisms. The first involves lowfrequency (frequency lower than the instability frequency in the flow) or steady energy addition to the flow, such as fluidic chevrons for noise mitigation [53]. The second mechanism involves direct manipulation of flow instabilities. This mechanism requires exciting the known flow instabilities (Section 2.1.3) by providing perturbation of the right frequency and mode.

For low Reynolds number flows, instabilities can easily be manipulated by introducing perturbations via acoustic drivers. Unfortunately, acoustic drivers lose control authority in high Reynolds number jets (typically Reynolds numbers greater than 100,000). The loss of control authority occurs because a high Reynolds number jet possesses a more energetic flow, which requires a greater input of energy to perturb the flow. Furthermore, instability frequencies in a laboratory flow are over a large bandwidth. Therefore, a new type of actuator that can operate in this environment is required.

Several types of actuators have been used to excite instabilities in high Reynolds number flows, including fluidic, piezoelectric and plasma actuators. Although both fluidic actuators [54] and piezoelectric actuators [55] are high amplitude, they are limited to narrow-band and lower frequencies. Localized Arc Filament Plasma actuators (LAFPA), which were developed at the Ohio State University, provide high amplitude and large bandwidth perturbations ideal for flow control of high-speed and high Reynolds 
number jets. GDTL has used these actuators for noise mitigation and flow control in a large range of Mach numbers and temperatures [15, 16, 20-24]. Each LAFPA consists of a pair of electrodes placed very near the nozzle exit, connected to a high voltage source through a switching circuitry. Closing the switch causes the voltage across the electrodes to rise until breakdown of the air between them occurs, which introduces perturbations to the flow. These perturbations excite the flow instabilities. Significant control authority on these flows has been demonstrated using LAFPAs with the aid of various measurement techniques (particle image velocimetry, schlieren imaging, flow visualization, and acoustic measurements in the flow-field, intermediate field, and far field). Furthermore, previous investigation techniques have revealed that forcing with LAFPAs has the effect of organizing the jet shear layer $[20,22,23,56]$. This means that large-scale structures are created in the flow with periodicity (in both time and azimuth) closely matching the excitation. LAFPA are used in the present work and further details of their operation will be described in the next chapter.

\subsection{Irrotational Near Field}

Valuable information regarding the nature of the large-scale flow dynamics of a subsonic jet can be obtained using measurements made in the near irrotational pressure field. If instability waves (or large-scale structures) are present in a turbulent jet, they must be accompanied by a convecting pressure field, which decays exponentially with radius in this region (as illustrated in the linear hydrodynamic regime in Figure 3) [26]. At the high frequencies, nonlinear pressure fluctuations, which are due to the smaller- 
scale turbulence, tend to decay more rapidly with radius. Furthermore, the acoustic waves generated by the turbulence decay more slowly but are likely to be of much smaller amplitude. The pressure signatures measured in the linear hydrodynamic region, which is also known as the irrotational near-field, can be considered to contain relatively local information concerning the large turbulence structures. Howes [57] was among the first to study the near pressure field of a subsonic jet. He observed that due to this very rapid decay of the fluctuating pressure level as a function of radial distance, the fluctuations at a certain near-field point are strongly determined by the turbulence in the flow immediately adjacent.

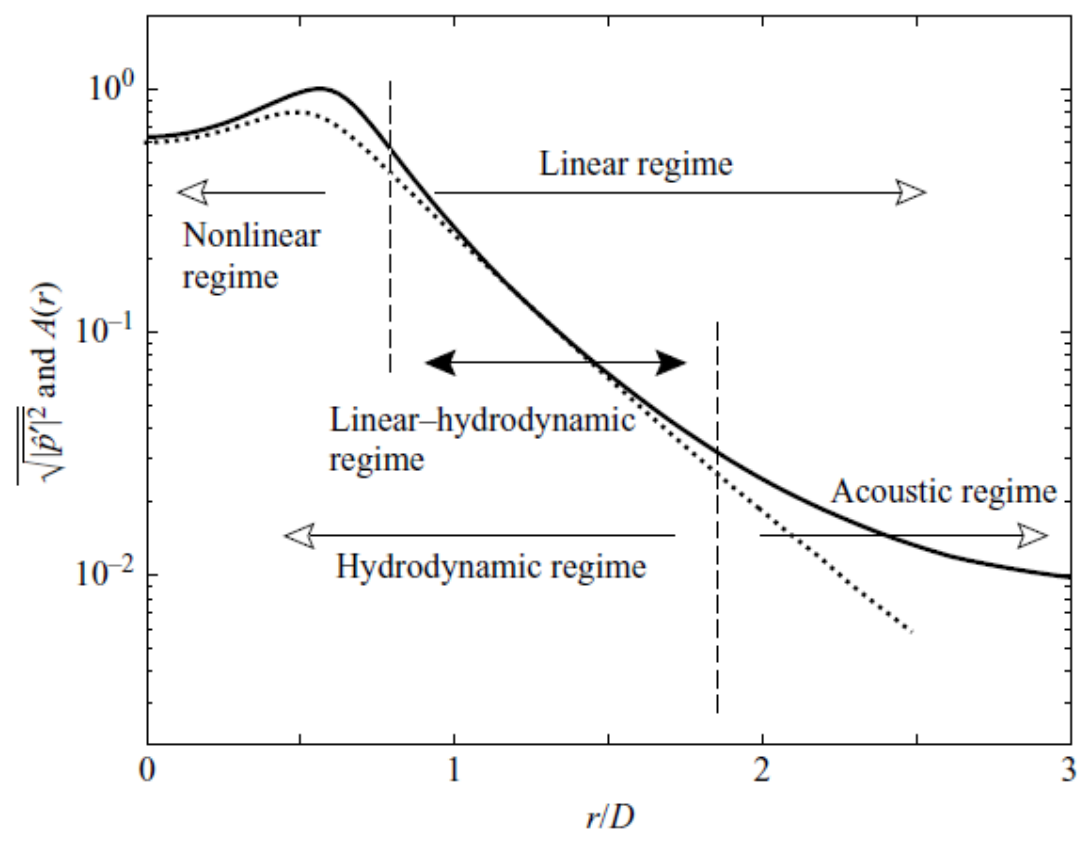

Figure 3. Diagram of the flow regimes of a round jet in the radial direction. The solid line depicts the time-averaged pressure fluctuation of the axisymmetric mode and the dotted line denotes the corresponding eigenfunction calculated from linear stability analysis [26]. 
There are numerous advantages to this measurement approach. For example, the technique is non-intrusive as it involves the use of microphones located just outside the jet periphery in the irrotational region. In addition, pressure is a scalar quantity which is considerably easier to measure than the velocity field inside the flow. There is, however, an uncertainty as to how much information is being filtered with respect to the pressure signatures in the turbulent flow. In addition to comprising a hydrodynamic signature of the turbulence, the near field also contains the contributions of the acoustic pressure field that radiate to the far field. Mollo-Christensen [58] points out that while the measurements obtained are related to hydrodynamic instabilities, part of what is observed corresponds to sound waves that propagate downstream in the jet. Thus, interpretations of near-field measurements in terms of the underlying large turbulence structure can be challenging, as it is difficult to determine the contribution of the acoustic fluctuations. 


\section{Chapter 3: Experimental Methodology}

\subsection{Jet Facility}

All experiments are conducted in the newly upgraded anechoic chamber at the GDTL within the Aeronautical and Astronautical Research Laboratories (AARL) at The Ohio State University. A schematic of the chamber is illustrated in Figure 4. The chamber is anechoic down to a cut-off frequency of $160 \mathrm{~Hz}$ provided by the fiberglass wedges. The design and validation of the chamber has been documented in detail [59].

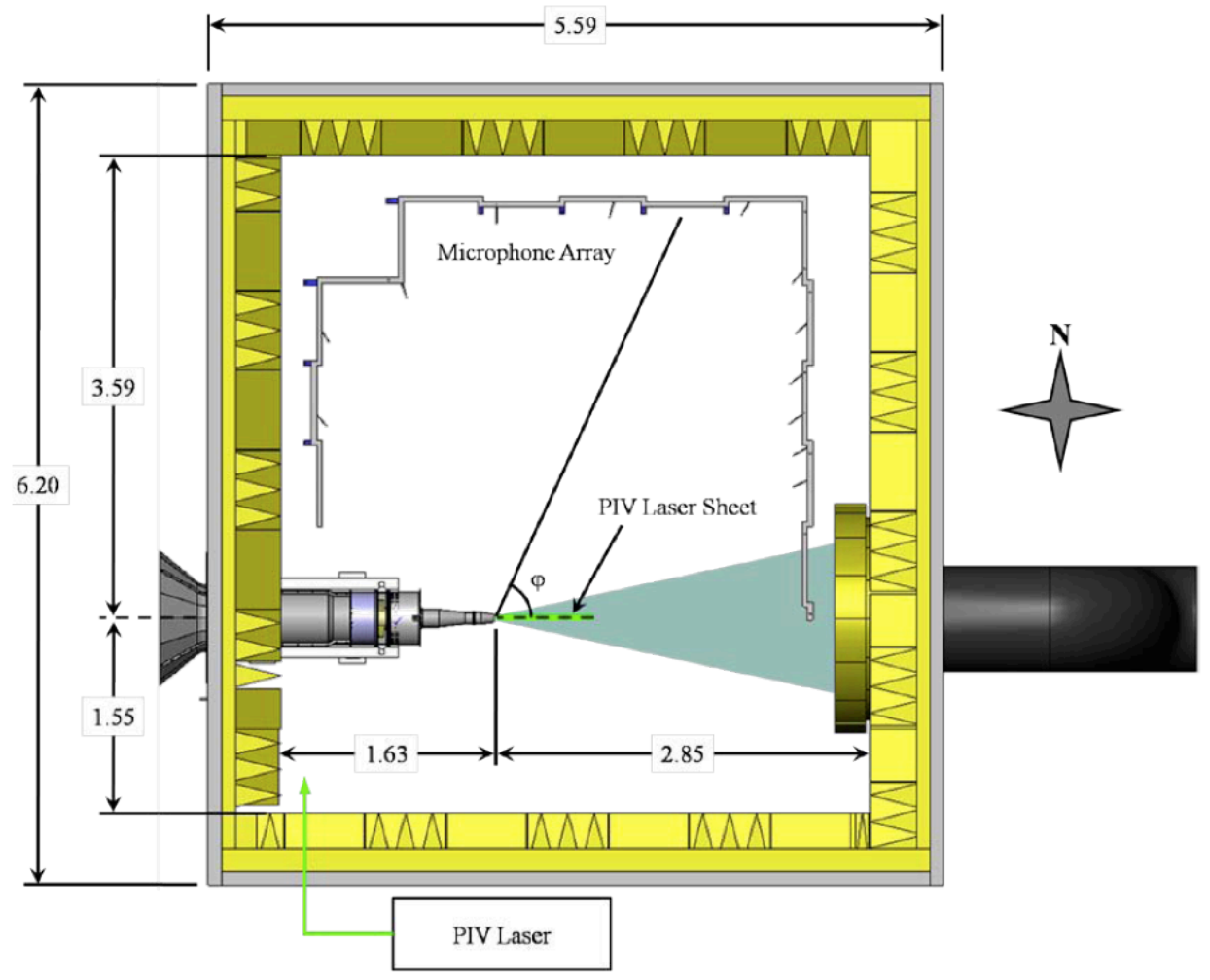

Figure 4. Schematic of anechoic chamber and jet. 
Compressed air, supplied with three 5-stage reciprocating compressors, is filtered, dried, and stored in two cylindrical tanks with a volume of $43 \mathrm{~m}^{3}$ and pressure up to 16 MPa. The compressed air is passed through a storage heater at a set temperature to heat the air to the desired temperature (the heater was not utilized in the current work), and then supplied to the stagnation chamber of the jet facility. The air is discharged horizontally through the nozzle into the anechoic chamber and then through an exhaust system to the outdoors.

The present work employs a stainless steel, axisymmetric nozzle with an exit diameter of $25.4 \mathrm{~mm}(1 \mathrm{in}$.). The subsonic jet is formed through a converging nozzle designed using a $5^{\text {th }}$ order polynomial for the internal contour. The nozzle is operated at a hydrodynamic Mach number of 0.9 with a Reynolds number based on jet diameter of approximately $6.2 \times 10^{5}$.

\subsection{Plasma Actuators}

As mentioned, flow control actuators known as localized arc filament plasma actuators have been developed in-house at the GDTL. Each actuator consists of a pair of pin electrodes. Tungsten rods, with a diameter of $1 \mathrm{~mm}$, are utilized as the electrodes. The electrodes are distributed around the nozzle extension perimeter, approximately $1 \mathrm{~mm}$ upstream of the nozzle exit plane, as shown in Figure 5(a). Measured center-to-center, the spacing between a pair of electrodes for each actuator is $4 \mathrm{~mm}$, and the distance between the neighboring electrodes of two adjacent actuators is $6 \mathrm{~mm}$. The electrodes are held in place by a nozzle extension made of boron nitride. A ring groove of $0.5 \mathrm{~mm}$ depth and 1 
$\mathrm{mm}$ width cut into the extension is used to house the electrodes and to shield the plasma [60]. With this arrangement, eight actuators are uniformly distributed around the nozzle extension so that the azimuthal spacing between two adjacent actuators is $45^{\circ}$.

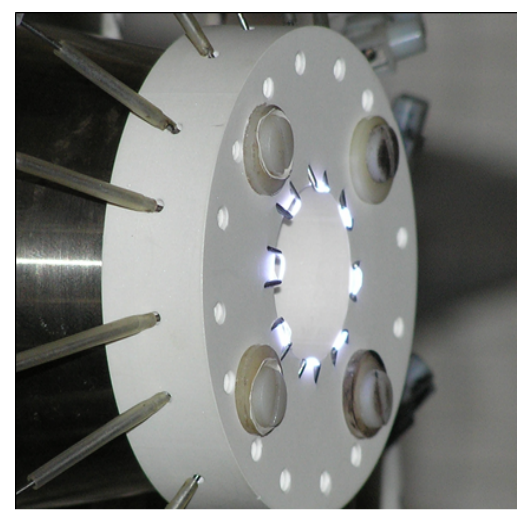

(a)

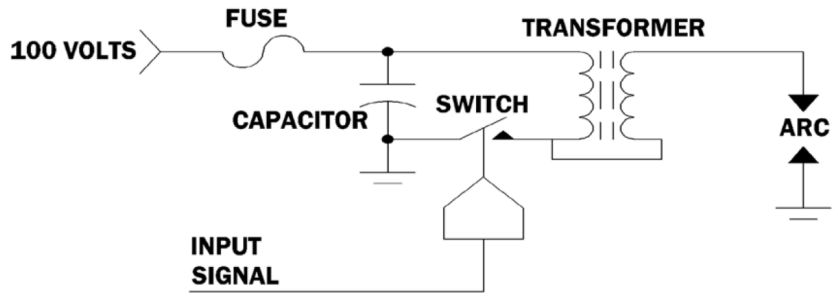

(b)

Figure 5. (a) LAFPAs housed in nozzle extension, (b) Schematic of high-voltage circuitry for one LAFPA channel.

A second-generation multi-channel high voltage power supply and plasma generator, designed and built at The Ohio State University, is utilized to power the plasma actuators. The plasma generator enables simultaneous powering of up to eight LAFPAs with independent frequency, duty cycle/pulse width, and phase control of individual actuators. Each individual circuit consists of a switchable capacitor in line with a high voltage transformer; the arcing electrodes are connected to the secondary side of the coil. The capacitor is charged by a $100 \mathrm{~V}$ DC power supply when the first switch is closed and the second is open; at the user-specified time the switches flip and it discharges through the coil. A schematic of the circuitry can be found in Figure 5(b). The switches are controlled 
by a 16-channel digital I/O card and National Instruments' LabVIEW software, operated by a dedicated computer. The pulse width was held constant at $7 \mu$ s, which was found to be the minimum pulse width at which the actuators consistently arced for all frequencies explored in this study [60]. This cycle can repeat to a maximum of 100,000 times per second $(100 \mathrm{kHz})$, though presently it is limited to only $20 \mathrm{kHz}$ by cooling requirements. More information on the construction and operation of the LAFPAs can be found in previous publications from the GDTL [20, 61-64].

\subsection{Data Acquisition}

\subsubsection{Near and Far Field}

The irrotational pressure field is acquired using a linear array of eight microphones in a meridional plane of the jet (as shown in Figure 6(a)). The linear microphone array is attached to a linear traverse and a rotation mount, both of which can be independently controlled. The separation between any two microphones is $25.4 \mathrm{~mm}$ (1 in). For the

present study, the most upstream microphone is placed at $x / D=2$, measured from the nozzle exit plane. The linear array is rotated such that the tips of the microphones form a line inclined at $8^{\circ}$ to the jet axis. The initial radial location of the most upstream microphone is $r / D=1.28$. This configuration ensures that the sensors are approximately equidistant from the outer edge of the shear layer of the unforced Mach 0.9 jet, as measured in earlier PIV experiments [21]. In the subsequent runs, the microphones are shifted in the radial direction in $5.08 \mathrm{~mm}(0.2 \mathrm{in}$.) increments for a total travel of $r / D=5$. A schematic of the microphone placements used is presented in Figure 6(b). 


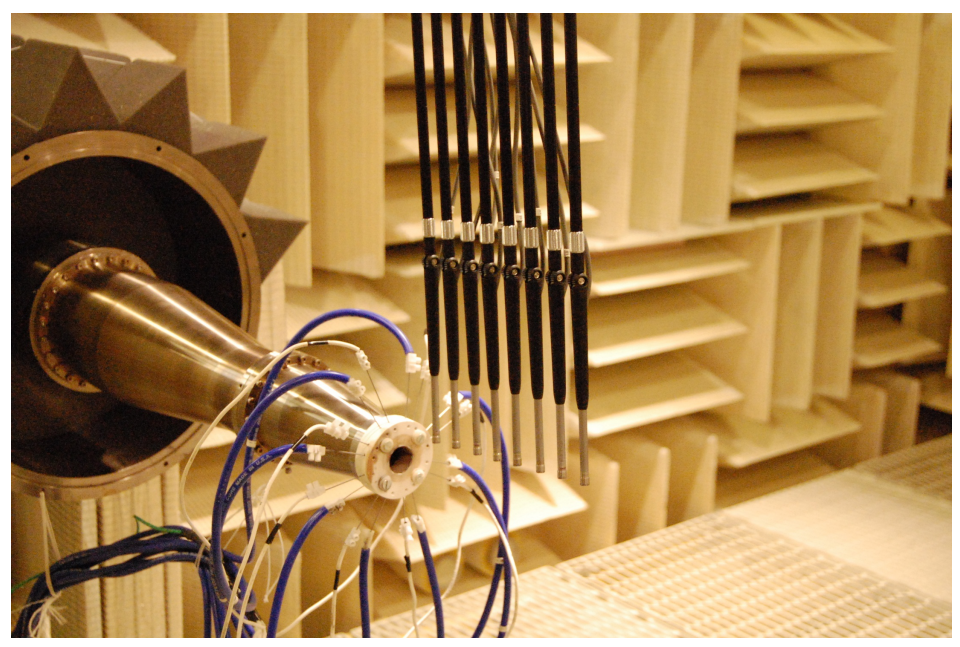

(a)

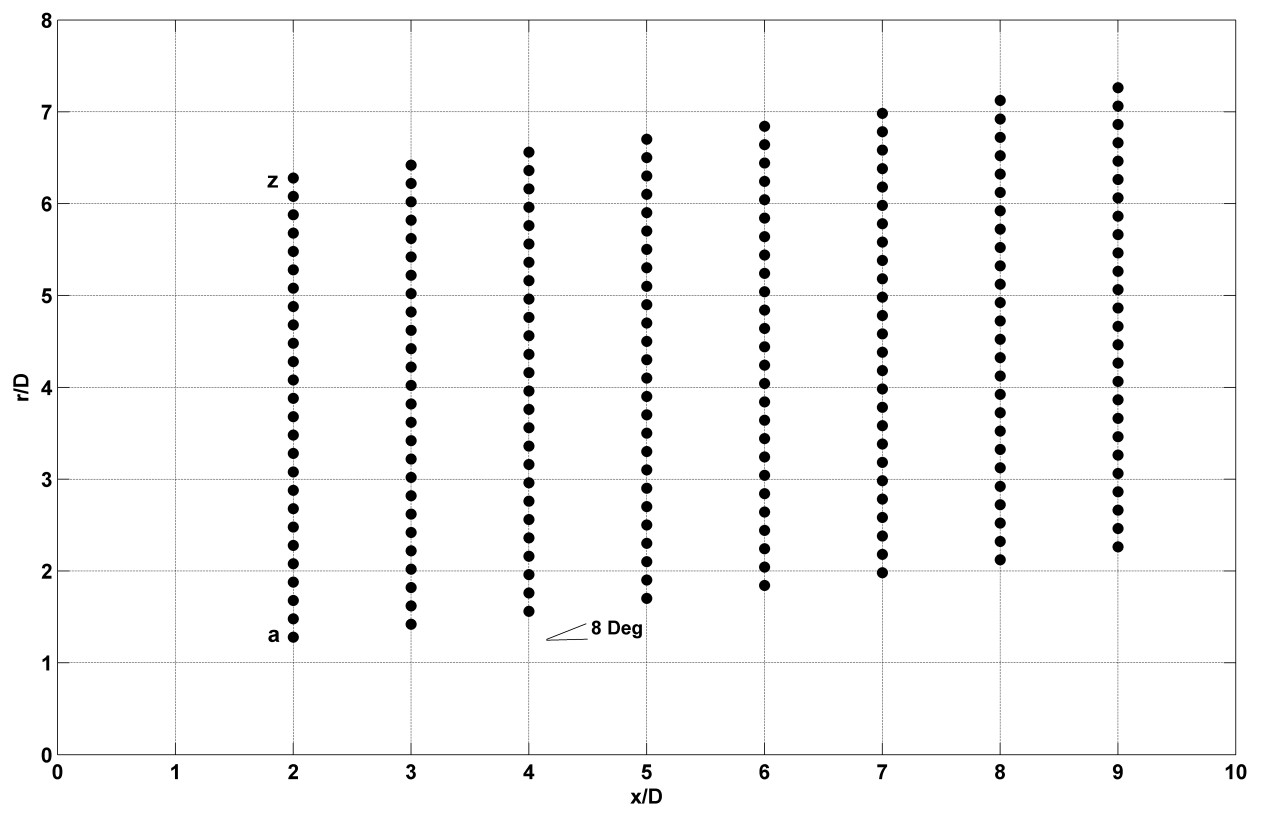

(b)

Figure 6. (a) Linear array for pressure measurements, (b) Microphone array grid coordinates for irrotational pressure field measurements.

Far-field acoustic pressure was simultaneously acquired at eleven polar angles spanning $25^{\circ}$ to $120^{\circ}$, as measured from the downstream jet axis. The far-field 
microphone array is comprised of two linear sections running perpendicular to the jet axis that contain the upstream and downstream angle microphones, and a third linear section running parallel to jet for the sideline angle microphones (Figure 4). The microphones are oriented such that the normal vector from their tips intersects the jet downstream axis at the nozzle exit. The radial distance of the microphones ranges from $96.5 \mathrm{D}$ at $25^{\circ}$ to $145 \mathrm{D}$ at $60^{\circ}$.

The microphones used are $1 / 4$ inch Brüel \& Kjær (B\&K) 4939. The voltage signal from each microphone is band-pass filtered from $20 \mathrm{~Hz}$ to $100 \mathrm{kHz}$, amplified by B\&K Nexus 2690 conditioning amplifiers, and simultaneously acquired using National Instruments PXI-6133 A/D boards and LabVIEW software. The microphones are calibrated using a $114 \mathrm{~dB}, 1 \mathrm{kHz}$ sine wave, and the frequency response of the microphones is flat up to $80 \mathrm{kHz}$ with the microphone grid cover removed. Signals are acquired at $200 \mathrm{kHz}$ with 81920 data points per block of samples. Ten such blocks of data are recorded for each experimental case producing a total sample time of about 4 seconds.

In this study, the jet is excited at a forcing frequency, in terms of Strouhal number, of $S t_{D F}=0.02$. This low forcing frequency was chosen such that the large-scale structures generated are convected downstream without any interaction with the subsequent or previous structures. The eight LAFPAs are operated in phase to simulate axisymmetric forcing $(m=0)$. 


\subsubsection{Actuation Phase}

The phase averaging technique used by Sinha et al. [28] is employed in order to study the evolution of the seeded perturbations. The actuators have control authority on the jet so that forcing results in a highly correlated fluctuation field - thereby justifying the application of phase averaging. Since the frequency and firing order of the actuators are specified by the user and held fixed by the controlling computer throughout an experiment, the only unknown information is the instantaneous phase. The pulse train controlling the first LAFPA is supplied to an arbitrary waveform generator (Agilent 3320A $20 \mathrm{MHz}$ ), where the rising edge on the transistor-transistor logic (TTL) pulse triggers a rising ramp signal over a time interval which is shorter than the forcing period. This ramp signal is acquired simultaneously with the near- and far-field pressure signals using the National Instruments hardware and LabVIEW program. This ramp signal is necessary in order to locate the zero phase of the actuator signal to sub-sample accuracy and to ensure that the short duration plasma pulses $(7 \mu \mathrm{s})$ can be robustly identified in potentially noisy data. Utilizing the known phase information, the pressure signals can be phase averaged to examine consistently occurring signal features. 


\section{Chapter 4: Unforced Jet Characteristics}

\subsection{Spectral Analysis}

\subsubsection{Far-Field Spectra}

The sound pressure level (SPL) plot for the Mach 0.9 jet is illustrated in Figure 7. The frequency is converted to the non-dimensional Strouhal number $S t_{D}=f D / U_{j}$. The figure gives far-field noise spectra acquired at eleven polar angles spanning $25^{\circ}$ to $120^{\circ}$ (direction as measured from the downstream jet axis as shown in Figure 2 of Section 2.1.2). As can be seen, the spectra possess shapes that are dependent on the observation angle. For example for the $30^{\circ}$ observation angle, the spectrum reaches a maximum at a Strouhal number of $\sim 0.17$, while for the $120^{\circ}$ observation angle, the spectrum is relatively broadband. The shape of these spectra can be linked to the source of their radiation. As explained in Section 2.1.2 on turbulence mixing noise, the radiation that reaches the $30^{\circ}$ observation angle comes from the large-scale turbulent structures while that of the upstream and sideline directions originates within scales that are smaller than the largest scales. Tam et al. [41] among others associate this radiation with small-scale structures (see Section 2.1.2). 


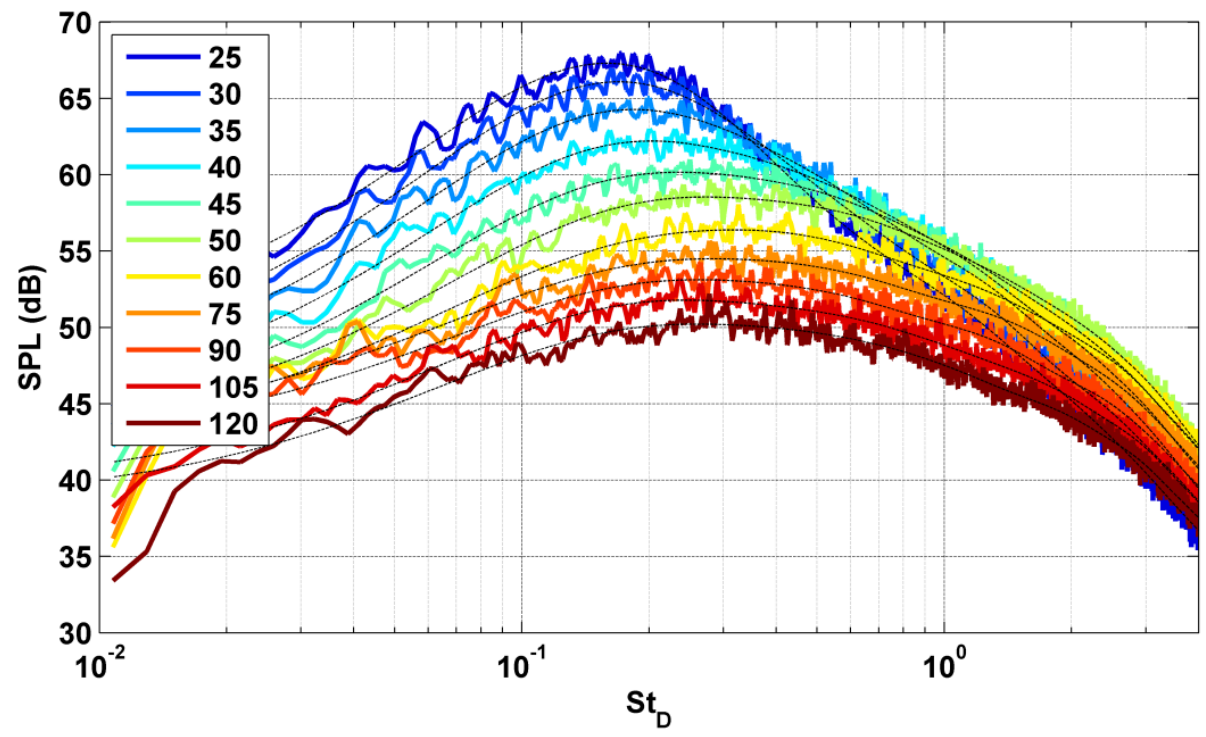

Figure 7. Far-field spectra of a Mach 0.9 axisymmetric jet.

\subsubsection{Near-Field Power Density Spectra}

A characterization of the near-field pressure in the unforced jet situates the subsequent discussion of the impulse response. One of the most well-characterized aspects of the near-field pressure is the demarcation between the hydrodynamic and acoustic signatures, which was first introduced by Arndt et al. [29] and later further established in numerous experimental papers [27, 30, 65]. Arndt et al. [29] showed that hydrodynamic fluctuations decay exponentially, while acoustic fluctuations decay algebraically.

The power spectral density (PSD) plots for the unforced jets are presented in Figure 8. The PSD here is normalized by the jet dynamic head $\rho_{j} U_{j}^{2}$, where $\rho_{j}$ is the jet exit density and $U_{j}$ is the jet exit velocity. This scaling is the established normalization for the near-field pressure fluctuation amplitude for cold subsonic jets [29, 30]. Figure 8(a) 
illustrates the PSD for the first measured microphone array position (label (a) in Figure 6(b)). As shown in Figure 8(a), increasing the axial distance leads to an overall shift of the peak towards lower Strouhal numbers. This behavior has been noted by previous researchers, and associated with the growth of large-scale structures in the shear layer [29]. Figure 8(b) shows the spectra for multiple radial locations at the same axial location $(x / D=5)$. As aforementioned, hydrodynamic fluctuations have been shown to decay exponentially, while acoustic fluctuations decay algebraically [29], so it can be inferred that the much stronger decay of the spectral peak in Figure $8(\mathrm{~b})$ is an indication of hydrodynamic fluctuation decay. Along the first array location (location (a) in Figure 6(b)), the pressure field is dominated by hydrodynamic fluctuations, whereas the acoustic component is more dominant at the last array location ((z) in Figure 6(b)). 


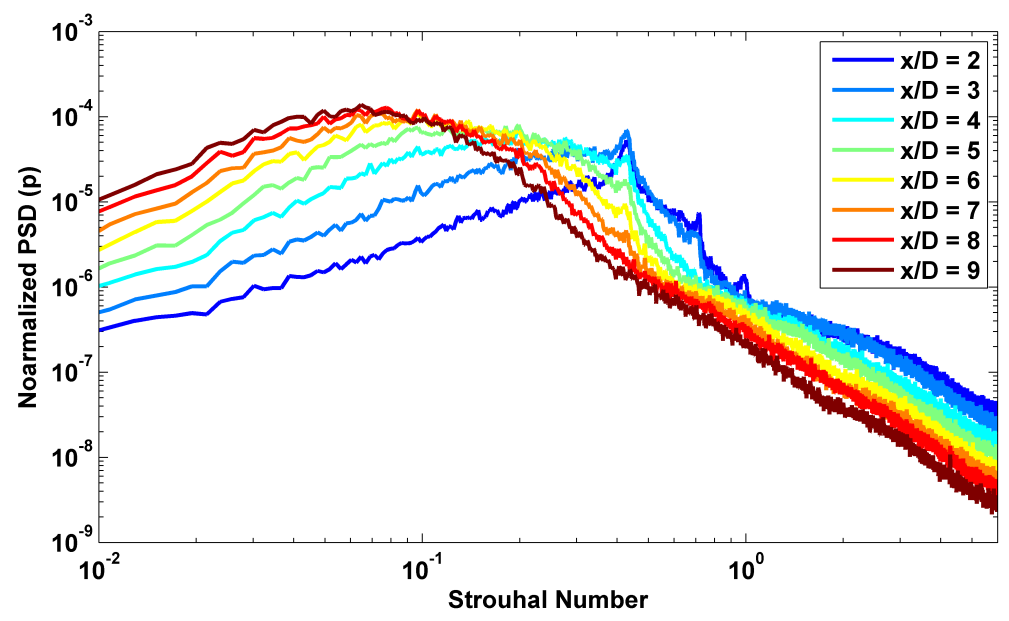

(a)

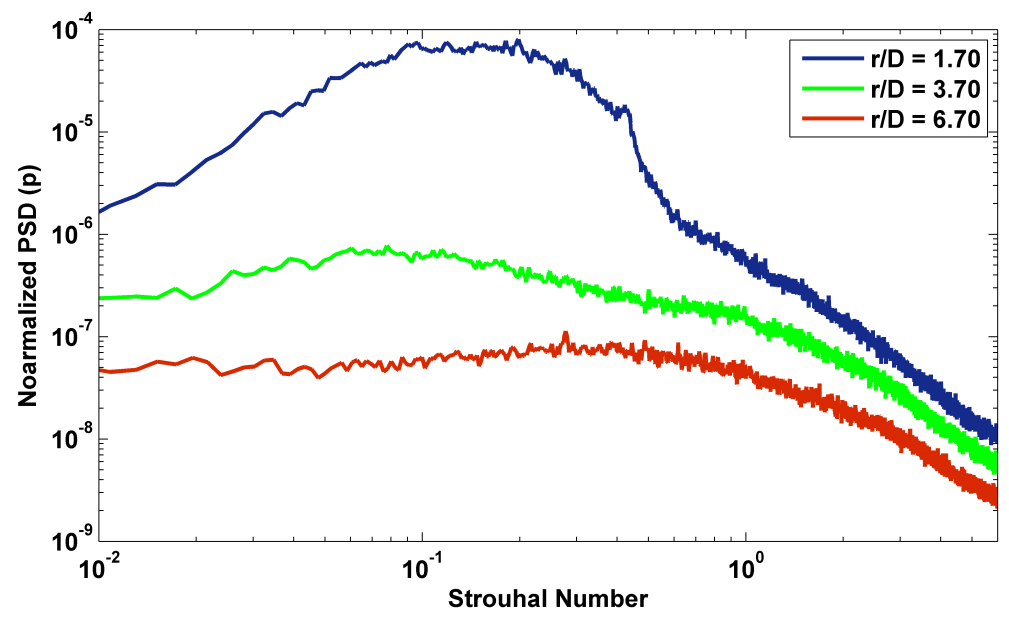

(b)

Figure 8. Power spectral density for unforced jet: (a) PSD for first array position at various axial locations, (b) PSD at $x / D=5$ for three radial locations.

A peculiar feature of the spectra in Figure 8(a) is the narrow-band peaks observed for the most upstream microphones at $S t_{D}=0.42$. Such a peak has been observed previously in our facility (though this was not always repeatable), and was suspected to be the result 
of a minor acoustic resonance in the jet plumbing. Given that the peaks are of relatively low amplitude, decay quickly as the jet evolves downstream, and are not observed in the far-field spectra, it is believed that our results are unaffected by their presence.

\subsubsection{Mean-Square of the Near-Field Pressure Fluctuations}

The simplest metric of the time-averaged response of the near-field pressure is the mean square (or equivalently, variance) of the pressure fluctuations, $\mathrm{p}_{\mathrm{MS}}$. The $\mathrm{p}_{\mathrm{MS}}$ in the unforced jet for the entire x-r domain is presented in Figure 9. It should be noted that the data is presented in a log scale. The growth and decay of the near-field pressure amplitude with axial distance has been well documented $[26,66,67]$. The near-field pressure is primarily hydrodynamic at the array locations closest to the shear layer. Thus, the behavior of $\mathrm{p}_{\mathrm{MS}}$ in the region closest to the shear layer is indicative of the corresponding dynamics of the large-scale structures that are evolving downstream. Furthermore, as can be seen in Figure 9, the near-field pressure amplitude rapidly decreases with increasing radial distance as has been previously shown in literature [26]. 


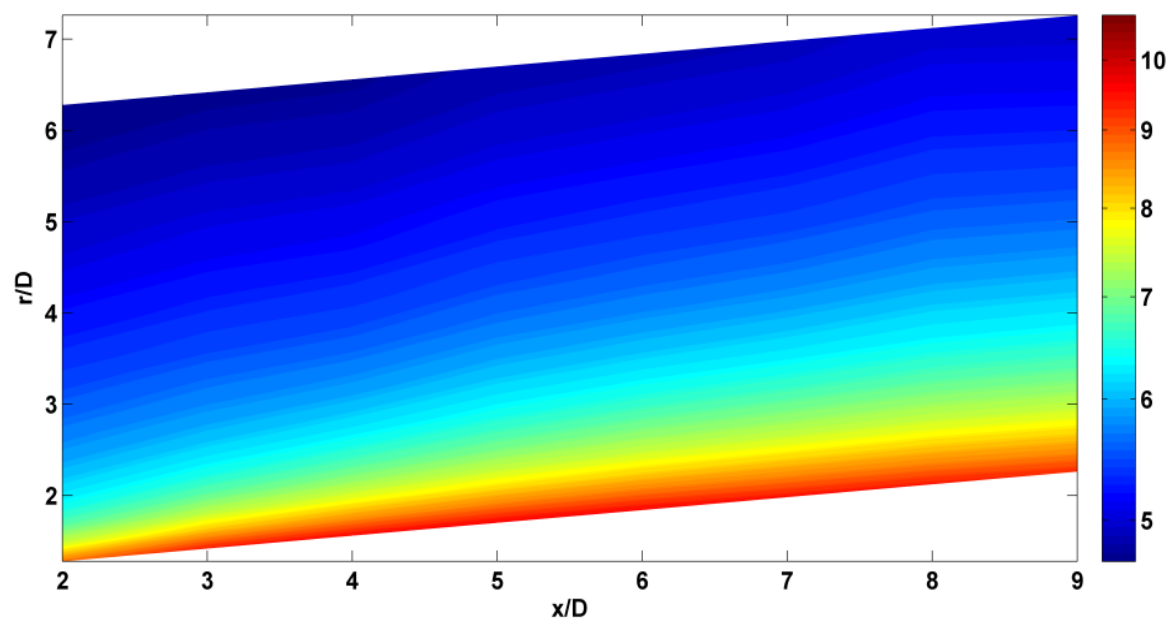

Figure 9. Mean-square of the near-field pressure fluctuations for unforced jet.

\subsection{Correlations}

\subsubsection{Autocorrelations}

Space-time correlation characteristics of the jet are investigated in order to gain insights into the physical mechanisms that lead to the generation and radiation of noise due to turbulent mixing in jets. Let $\mathrm{p}_{\mathrm{n}}(\mathrm{t})$ be the time dependent pressure signal measured by the $n^{\text {th }}$ microphone. The normalized autocorrelation, $R_{n n}(\tau)$, is defined as,

$$
R_{n n}(\tau)=\frac{\left\langle p_{n}(t) p_{n}(t+\tau)\right\rangle}{\left\langle p_{n}^{2}(t)\right\rangle}
$$

where, $<>$ means ensemble or time average. The normalization is such that the autocorrelations at zero lag are identically 1.0; this normalization will be used for all correlation plots to follow. Figure 10 illustrates the normalized autocorrelations for the jet at $x / D=6$ (Figure 10(a)) and $x / D=9$ (Figure $10(\mathrm{~b})$ ) for various radial locations. The sound field of a jet can be viewed as consisting of random acoustic pulses each with a 
spatial dimension. If the size of the acoustic pulse is small, then the normalized autocorrelation of such an acoustic field would have the shape of a spike with a narrow half-width. This is due to the fact that the signal is random and uncorrelated. It is reasonable to expect the size of the acoustic pulses from small-scale turbulent structures to be small given that these structures have been found to be uncorrelated with each other; for this reason, the normalized autocorrelation measured in the sideline directions of the jet is expected to have narrow half-widths. It is also reasonable to expect the size of the acoustic pulses radiated by the large-scale structures to be much larger; say, similar size to that of the large turbulence structures. Hence the half-width of the normalized autocorrelations measured near the jet shear layer and downstream of the nozzle exit would be correspondingly wider. This is visibly shown in Figure 10. As can be seen in Figure 10 (a) and (b), as the microphone is placed radially away from the jet, the halfwidth of the autocorrelations become narrower. This trend is also observed when the microphone is placed closer to the nozzle exit (from $\mathrm{x} / \mathrm{D}=9$ to $\mathrm{x} / \mathrm{D}=6$ ). In addition to that, the half-widths of the various radial locations are distinctly different; the autocorrelations of the array locations closest to the jet shear layer are characterized by very large and deep negative peaks. This observation suggests that an additional effect of the presence of large-scale structures is the deepening of the negative peaks in the autocorrelation function. Other researchers previously noted these observations as well [68]. 


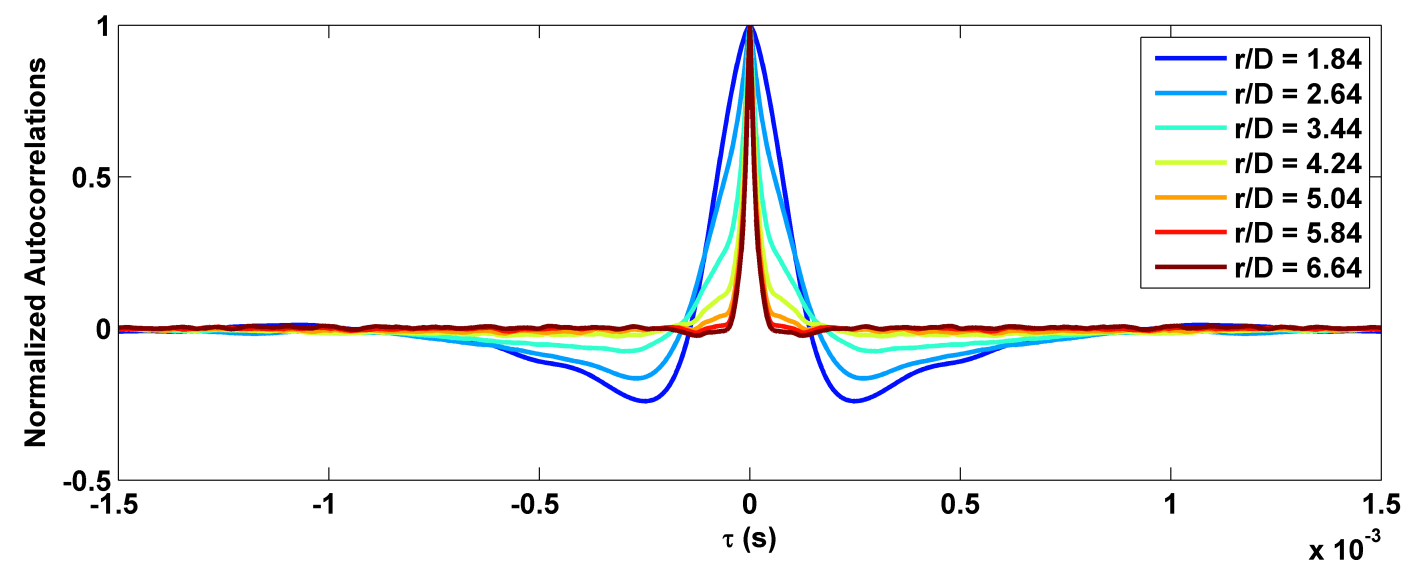

(a)

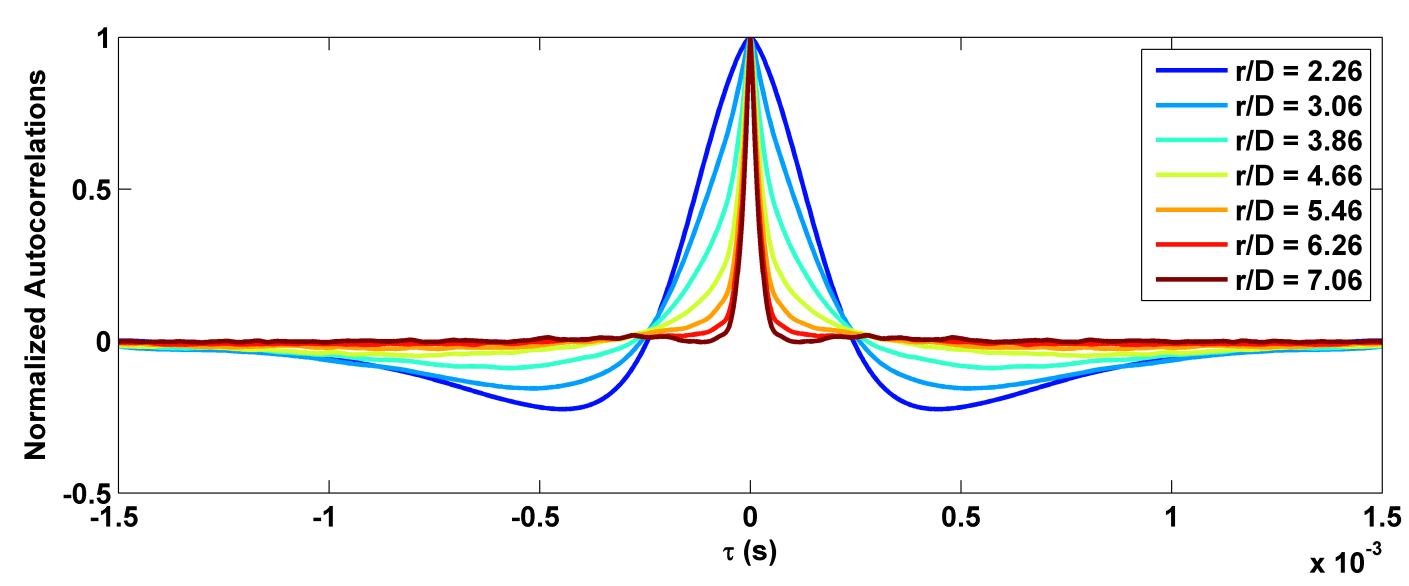

(b)

Figure 10. Normalized autocorrelations of the unforced jet at the same axial position and various radial locations: (a) $x / D=6$, (b) $x / D=9$.

\subsubsection{Near Field Cross-Correlations}

Microphone pressure cross-correlations were measured in order to obtain spatial information regarding the near field of a jet. If $\mathrm{p}_{\mathrm{m}}(\mathrm{t})$ is the time dependent pressure signal of the $m^{\text {th }}$ microphone and $p_{n}(t)$ is that of the $n^{\text {th }}$ microphone, then the normalized crosscorrelation, $\mathrm{R}_{\mathrm{mn}}(\tau)$, is defined as, 


$$
R_{m n}(\tau)=\frac{\left\langle p_{m}(t) p_{n}(t+\tau)\right\rangle}{\left\langle p_{m}^{2}(t)\right\rangle^{1 / 2}\left\langle p_{n}^{2}(t)\right\rangle^{1 / 2}}
$$

The normalized cross-correlations of the near-field pressure with the reference microphone at $x / D=9$ (last microphone in the array) are presented in Figure 11. Figure 11(a) illustrates the first radial location where the pressure field is dominated by hydrodynamic fluctuations. Little correlation is observed when the microphone is placed greater than $5 D$ from the reference microphone. The correlation increases with decreasing distance between the microphone and the reference microphone reaching a maximum of approximately 0.63 when the sensor is $1 D$ from the reference microphone. Although not shown in this thesis, similar correlations were acquired for various reference microphones $(x / D=2$ and $x / D=6)$ and similar levels of correlation were observed with the only difference being the inversion of the correlation shapes before and after the reference microphone which is to be expected. Slightly lower maximum correlation values are observed for the last array position (Figure 11(b)). Furthermore, note nearly an order of magnitude change in the time lag, indicating that this is due to the radiation from the acoustic fluctuations rather than the hydrodynamic fluctuations. One of the major differences between the two array locations presented is that there are no strong negative excursions for the last array location. These dissimilarities can be observed between the autocorrelation functions as well (Figure 10), and are the indication of the lack of presence of the convective signatures from the large-scale structures. 


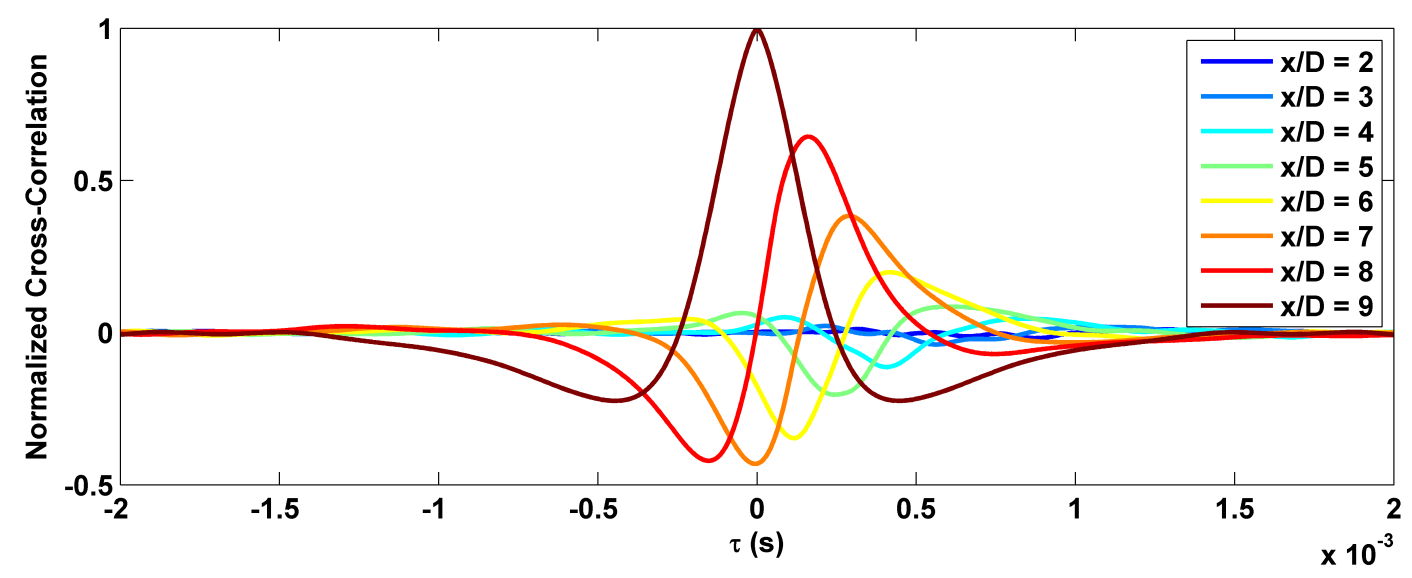

(a)

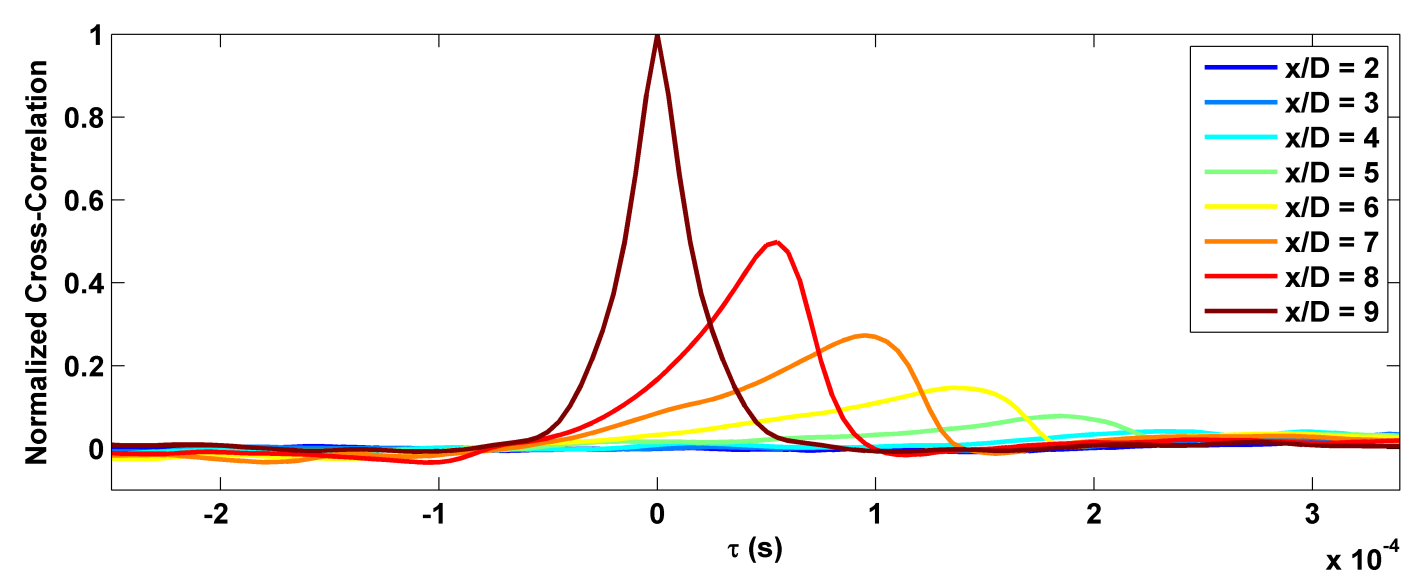

(b)

Figure 11. Normalized cross-correlations of the near-field pressure fluctuations for the unforced jet with the reference microphone at $x / D=9$ : (a) First array location, (b) Last array locations.

\subsubsection{Near Field to Far Field Cross-Correlations}

\subsubsection{Individual Array Locations}

The variation of the normalized cross-correlations of the near-field pressure along the first array position and last array position with the far-field microphone at $30^{\circ}$ are shown in Figure 12(a) and (b), respectively. As evident from Figure 12(a), the correlation 
increases as $x / D$ increases and the structures in the jet evolve, reaching a maximum of roughly 0.2 at $x / D=9$, which is the last microphone location in the array. This is consistent with previous researchers who have shown that the correlations are strongest downstream of the end of the potential core $[48,69]$, which occurs at roughly $x / D=6$ for this jet [21]. Given that the maximum correlation is occurring at the end of the measurement window, this metric should be further evaluated in future studies by extending the measurements further downstream. In addition to the maximum amplitude, the shape of the correlations evolves as the microphones are traversed downstream, which is more clearly illustrated in Figure 13 for the first array position. For example, at $x / D=5.0$ (Figure 13), the correlation curve is roughly symmetric: it has one large amplitude negative peak, bookended by two lower amplitude positive peaks (similar to the shape of the Mexican hat wavelet). Upstream of this position, the earlier positive peak has greater amplitude than the later one, while downstream of this position the later positive peak is dominant and the negative peak starts to gradually decrease. This trend in the shape of the correlation for the first array position is dissimilar to the last array location in that there is a sustained growth for both the positive and negative excursions (Figure 12(b)). It has been shown that the large-scale structures radiate predominantly downstream towards shallow angles to the jet, hence the observed growth with downstream distance for the last array. Additionally, the last array location reaches a maximum of roughly 0.15 , which is less than that observed in Figure 12(a). 


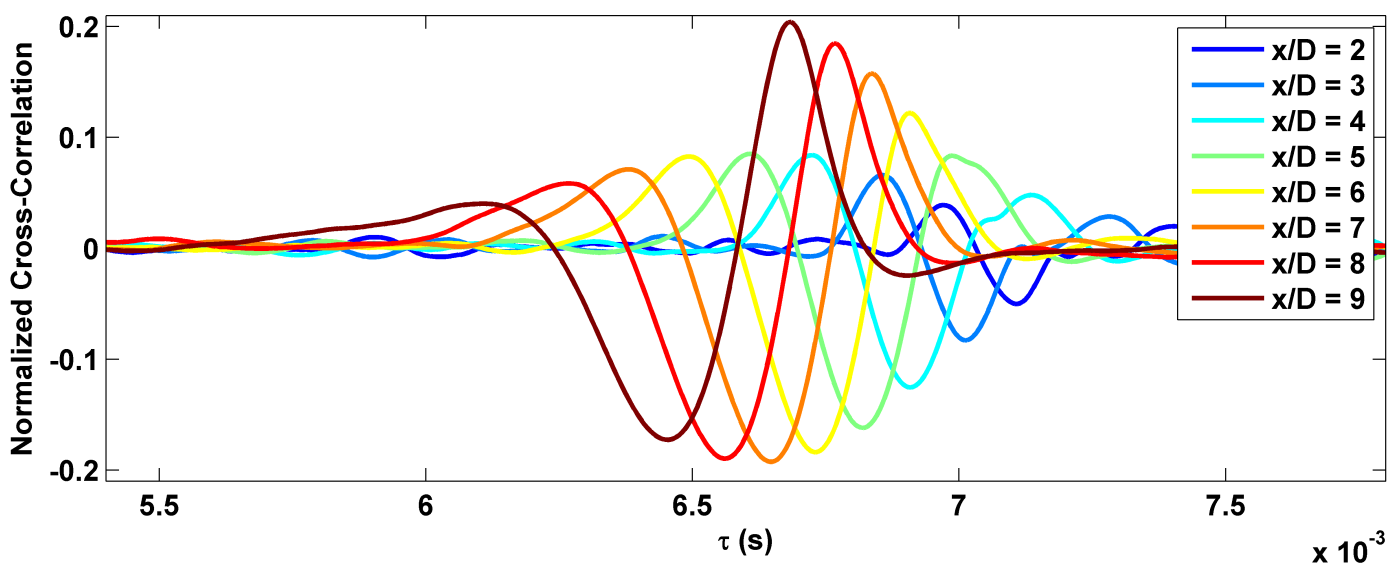

(a)

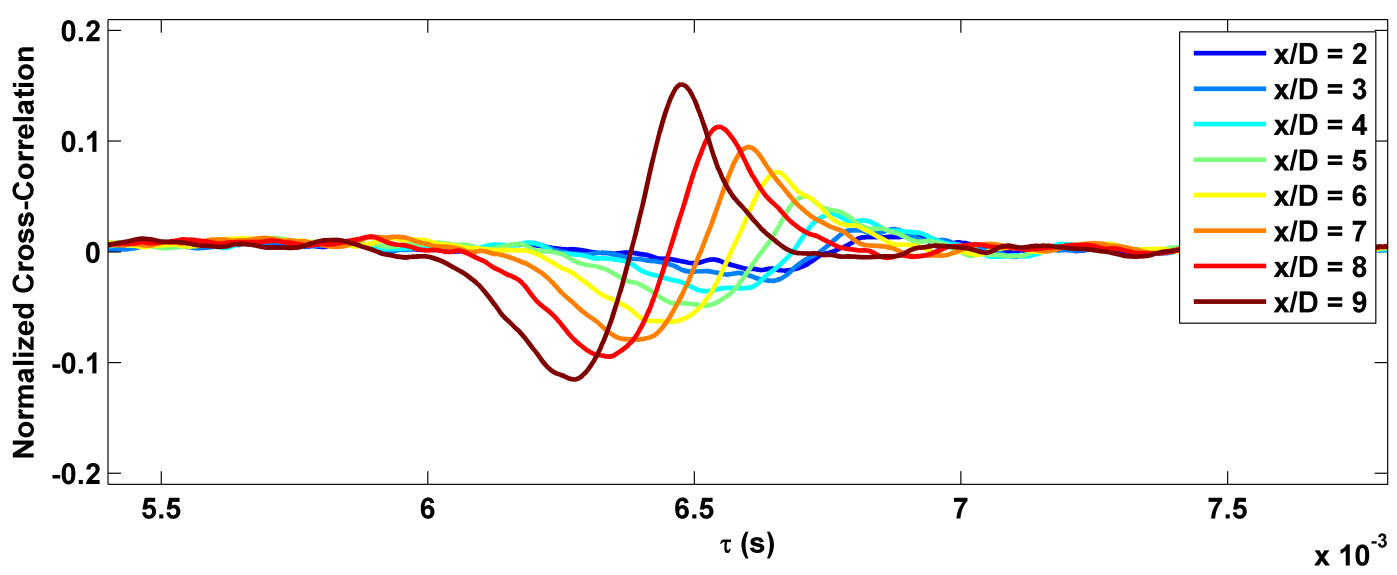

(b)

Figure 12. Normalized cross-correlation of the near-field pressure fluctuations for the unforced jet with the $30^{\circ}$ far-field microphone: (a) First array location, (b) Last array location. 


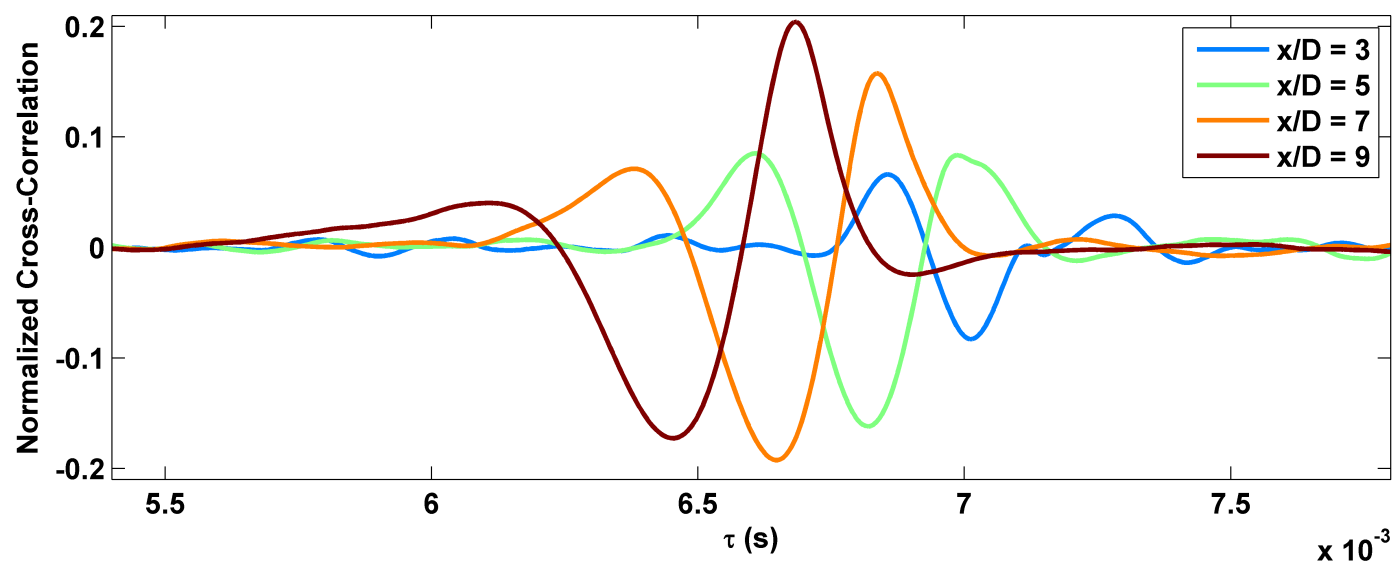

Figure 13. Normalized cross-correlation of the near-field pressure fluctuations for the unforced jet with the $30^{\circ}$ far-field microphone for the first array location.

\subsubsection{Maximum Correlation Maps}

In Figure 14, the maximum cross-correlation values between the near-field microphone signals and the far-field microphone signal at a given polar angle are plotted as a function of spatial coordinates of the near-field microphones. The maximum correlation is clearly a function of both axial and radial positioning of the near-field microphone. For the upstream microphone positions, particularly at large radial distances, the maximum correlation to $30^{\circ}$ far field (Figure $14(\mathrm{a})$ ) is virtually zero. This is expected given that the acoustics associated with the large-scale structures have been shown to radiate predominantly to the downstream angles [1]. The greatest correlation values are observed in the downstream direction, at an approximate angle of $22^{\circ}$ with respect to the jet exit. It is to be noted that this angle is dependent on the chosen viewing window for the maximum correlation and therefore is not an absolute number. Compared to the correlations at $30^{\circ}$, the correlations to $60^{\circ}$ (Figure $14(\mathrm{~b})$ ) are of significantly lower 
amplitude, and peak at a higher angle (approximately $45^{\circ}$ ). This is consistent with the concept that the largest contribution to the sideline angles is due to small scale, high frequency fluctuations, in contrast to the downstream angles, which are associated with large-scale structures. The region of high correlation relative to its surroundings is much broader at this polar angle than at $30^{\circ}$. The most upstream region has very low correlation to the acoustic far field at $60^{\circ}$ (again, as expected), however in this case the region close to the jet shear layer also shows low correlations to the $60^{\circ}$ far-field microphone. 


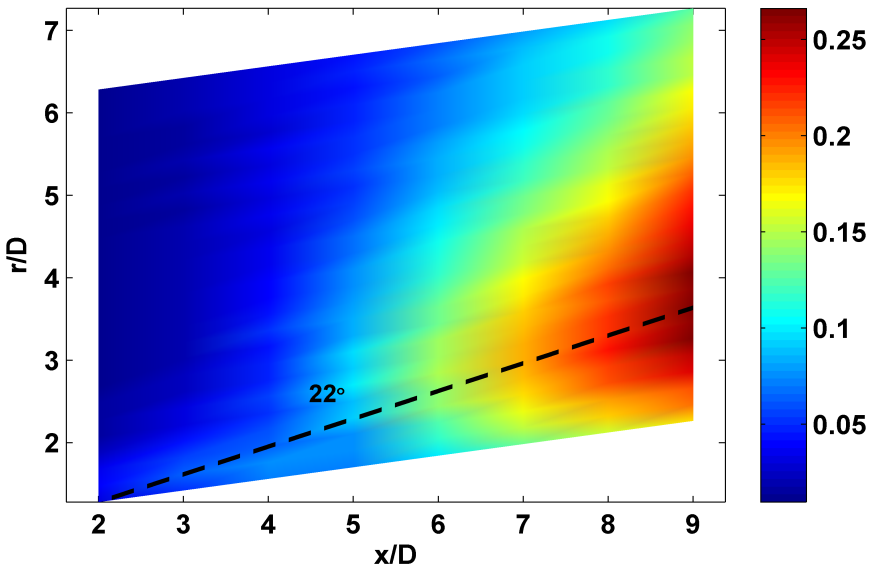

(a)

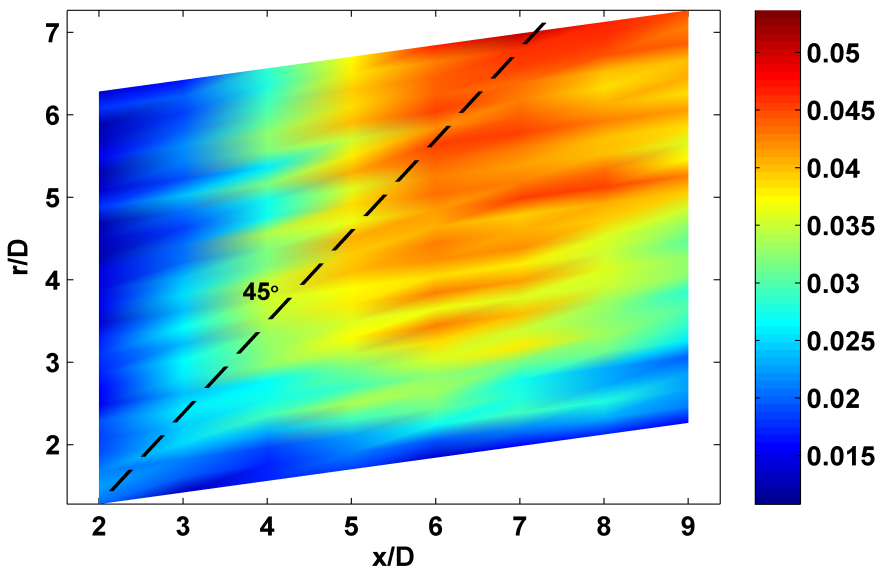

(b)

Figure 14. Maximum normalized cross-correlations of the near-field pressure fluctuations for the unforced jet: (a) With $30^{\circ}$ far-field microphone, (b) With $60^{\circ}$ far-field microphone. 


\section{Chapter 5: Effects of Forcing: Statistical}

\subsection{Spectral Analysis}

\subsubsection{Far-Field Spectra}

The SPL at $\theta=30^{\circ}$ when exciting the axisymmetric mode at a $S t_{D F}$ of 0.02 (corresponding to a frequency of approximately $250 \mathrm{~Hz}$ ) is presented in Figure 15 . The forcing frequency is normalized to the forcing Strouhal number $S t_{D F}=f_{F} D / U_{j}$. The baseline Mach 0.9 jet is also presented for comparison. The two spectra are similar apart from the presence of tones at the forcing Strouhal number (as indicated by the triangular marker in the figure) and its harmonics. It has been previously shown that the $S t_{D F}$ also plays an important role in determining the broadband amplitude effects [20]. It was found that the broadband amplitude increases most when forcing around the jet column instability $\left(S t_{D F}=0.36\right)$ and it decreases for especially high $S t_{D F}\left(S t_{D F}>1.08\right)$. However, due to the low $S t_{D F}$ used in this study, the broadband amplitude does not appear to have varied between the forced and unforced spectra. The reason for this will become clear soon, but it is due to the low forcing Strouhal number used and therefore the sparse presence of structures due to forcing. 


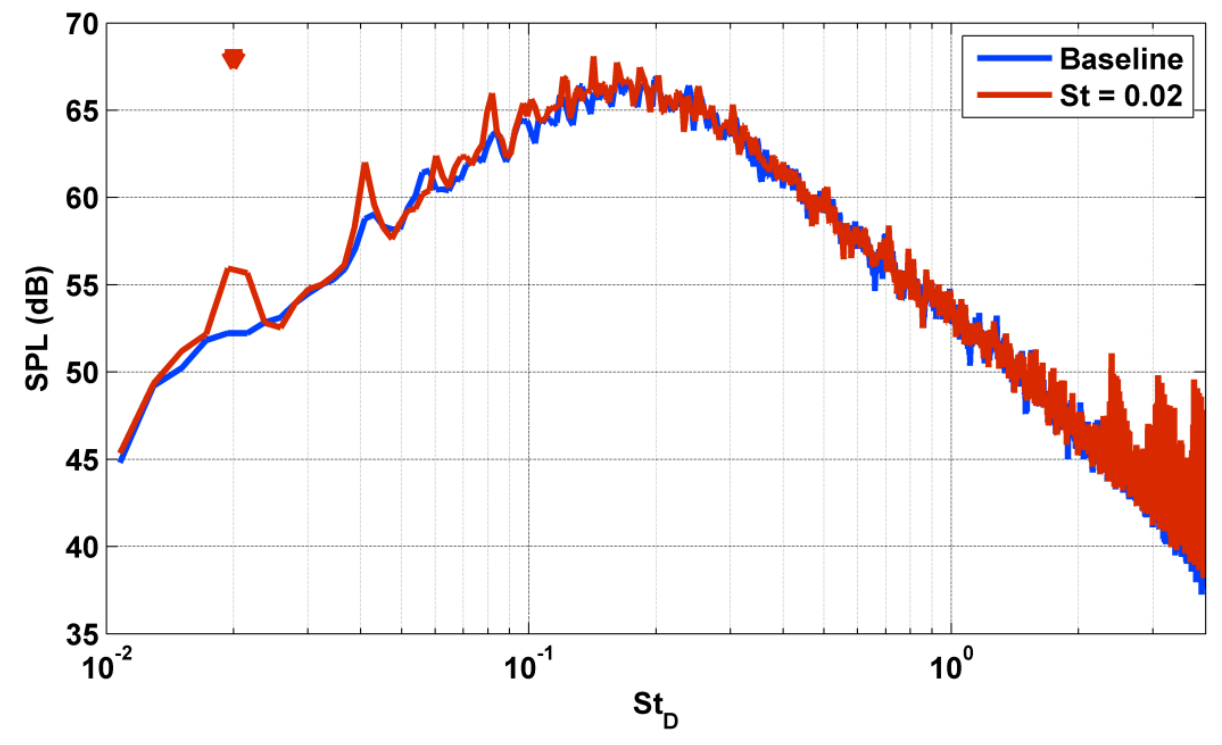

Figure 15. Far-field SPL at $\theta=30^{\circ}$ for the Mach 0.9 jet forced with $\mathrm{m}=0$ at $S t_{D F}=0.02$.

\subsubsection{Mean-Square of the Near-Field Pressure Fluctuations}

The forcing response of the jet is characterized using the near-field pressure measurements. The linear array of near-field probes is held fixed at the locations mentioned earlier. The forcing frequency is again normalized to the forcing Strouhal number. Previous research [28] has shown that when forcing the jet, each actuator impulse creates a flow perturbation that develops into a large-scale structure and convects downstream. This can be further exemplified by looking at the $p_{M S}$ of the forced fluctuations (Figure 16). Forcing the jet at $S t_{D F}=0.02$ causes the growth and decay of the near-field pressure amplitude to move upstream closer to the nozzle exit in comparison with the unforced jet case (Figure 9) with a maximum between $x / D=2.5$ to 5.5 . This is inconsistent with other results that will be discussed in the next few sections showing that 
forcing at this low Strouhal number does not change the statistics of the jet; the reason for this behavior is still undetermined.

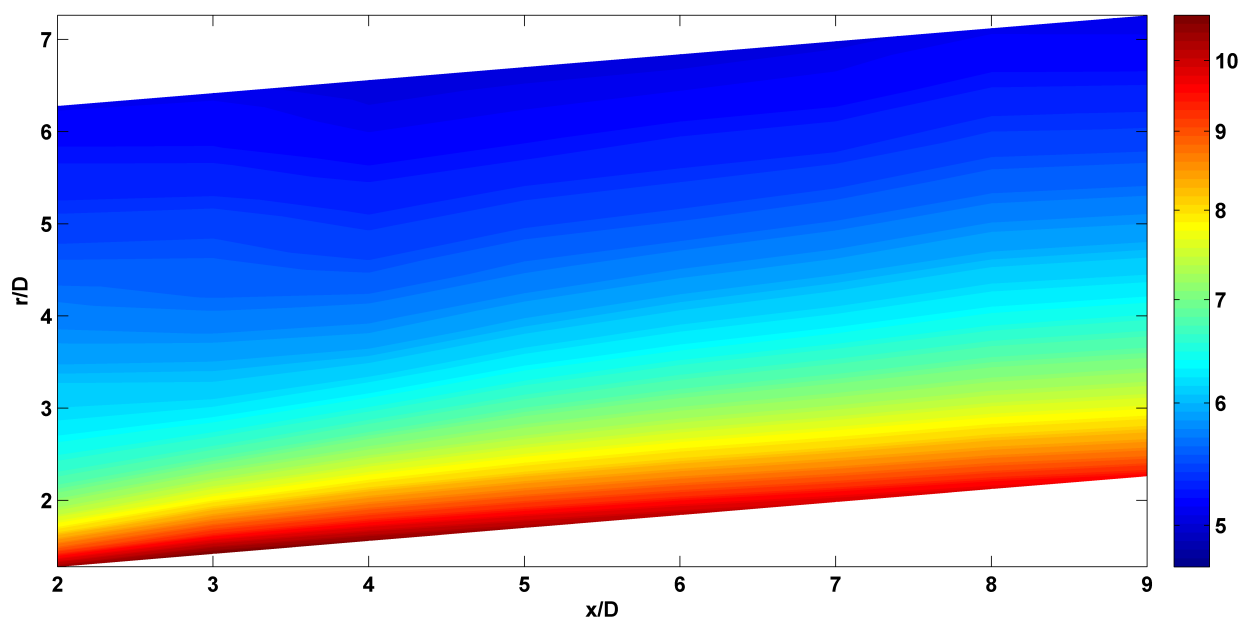

Figure 16. Mean-square of the near-field pressure fluctuations for the jet force at $S t_{D F}=0.02$.

\subsection{Correlations}

\subsubsection{Autocorrelations}

The normalized autocorrelations of the near-field pressure for the forced jet at $x / D=6$ and $x / D=9$ for various radial locations (similar to Figure 10) are presented in Figure 17. Immediately, it is evident that the autocorrelations for the forced jet are similar to those of the unforced jet. As before with the unforced jet, the half-widths of the autocorrelation functions become narrower as you move away from the jet shear layer and/or upstream closer to the nozzle exit indicating radiation due to the small-scale structures. Furthermore, the presence of large-scale structures is observed in the first few array locations by the large negative peaks. A closer look at the similarities between the unforced and forced cases are illustrated in Figure 18 for $x / D=9$ at the first array 
location. In this figure, only the half-width is shown since the function is symmetric, furthermore, this similarity is seen for all array and axial locations. As can be seen forcing the jet at $S t_{D F}=0.02$ results in a similar function to the unforced jet, this is due to the very low forcing frequency used and thus the sparse presence of additional structures due to forcing.

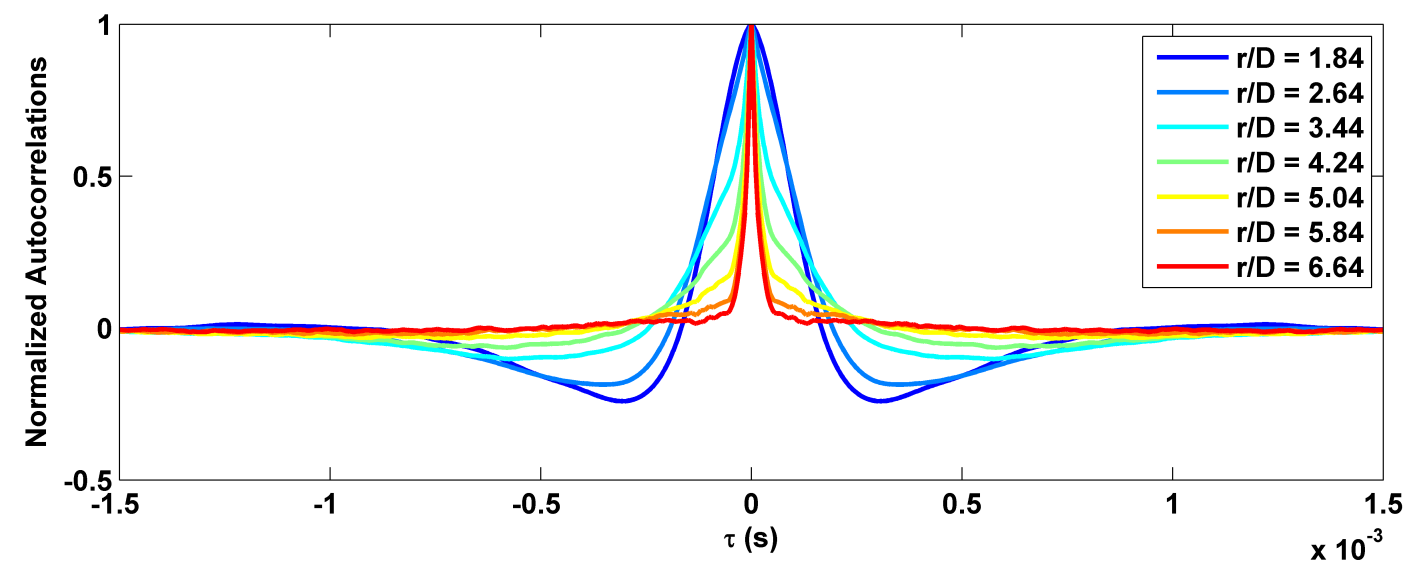

(a)

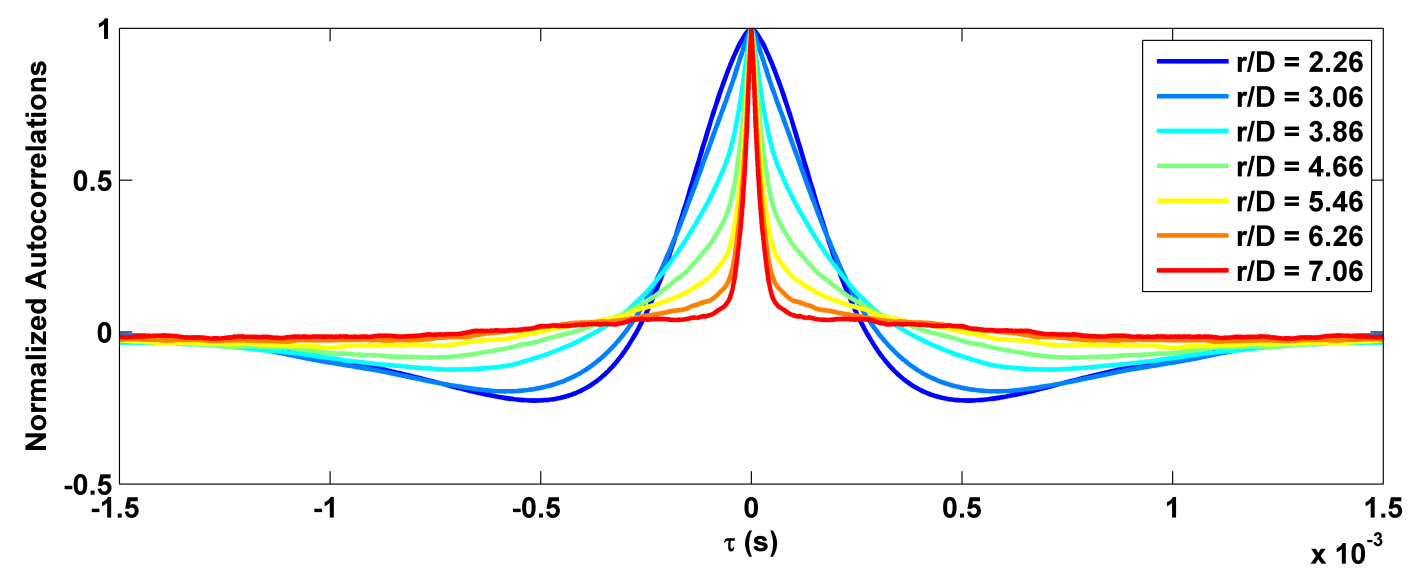

(b)

Figure 17. Normalized autocorrelations of the forced jet at the same axial position and various radial locations: (a) $x / D=6$, (b) $x / D=9$. 


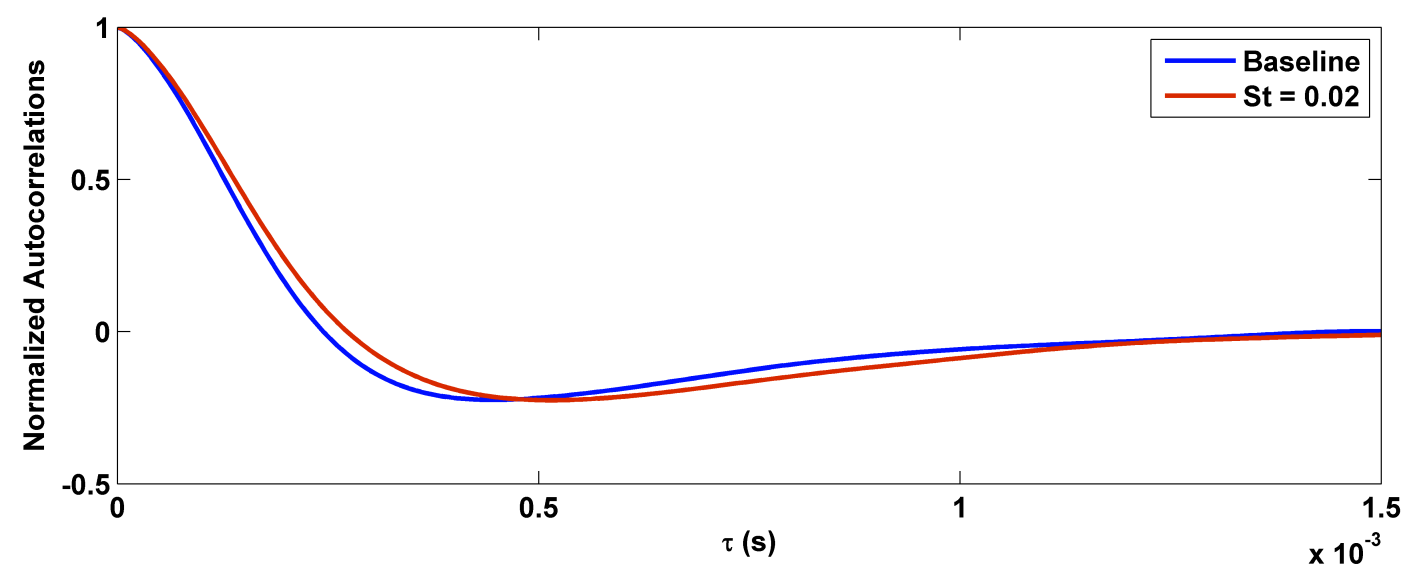

Figure 18. Normalized autocorrelation of the forced jet for $x / D=9$ at the first array location.

\subsubsection{Near Field Cross-Correlations}

The normalized cross-correlations of the near-field pressure for the forced jet with the reference microphone at $x / D=9$ (similar to Figure 11) are presented in Figure 19. Note that the scale (level and range) is varied between Figure 19(a) and (b) in order to better examine the trends observed. Unlike Figure 11(a) where roughly no correlation is observed for the upstream microphones in the first array location, correlation (although small) is visible over the entire axial extent for the forced jet. This is most likely due to the additional structures, although sparse, that come about due to forcing. Furthermore, the correlation shapes for both array locations are similar to that observed for the unforced jet (Figure 11), as was the case with the autocorrelations. 


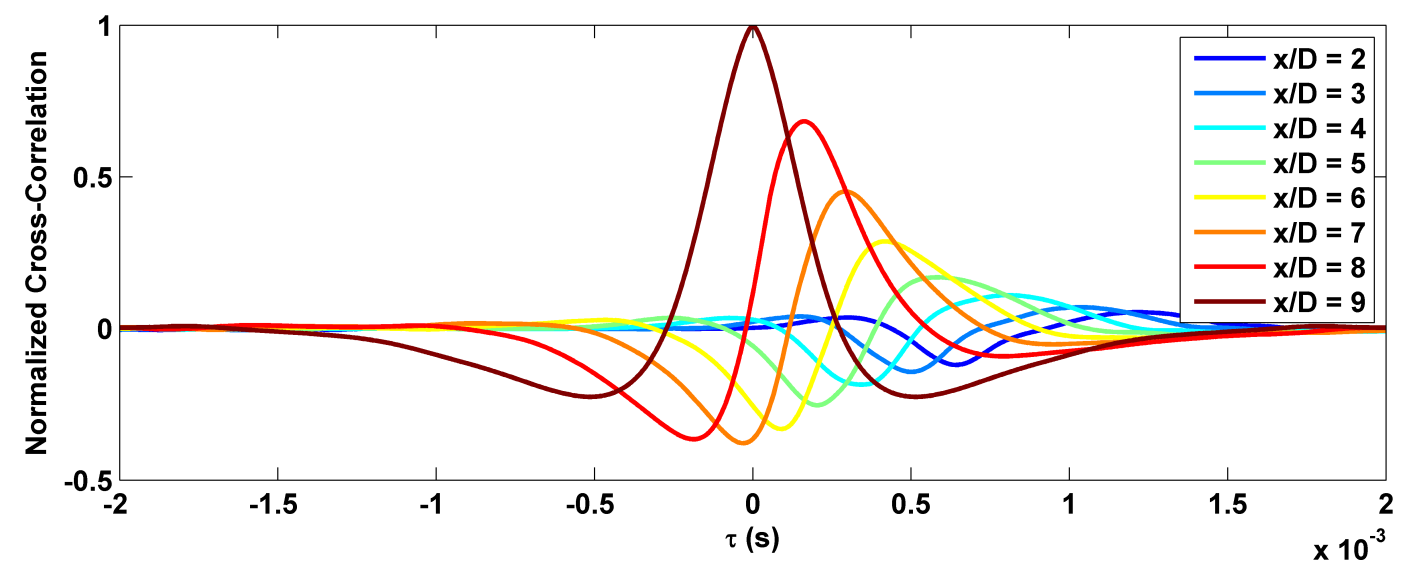

(a)

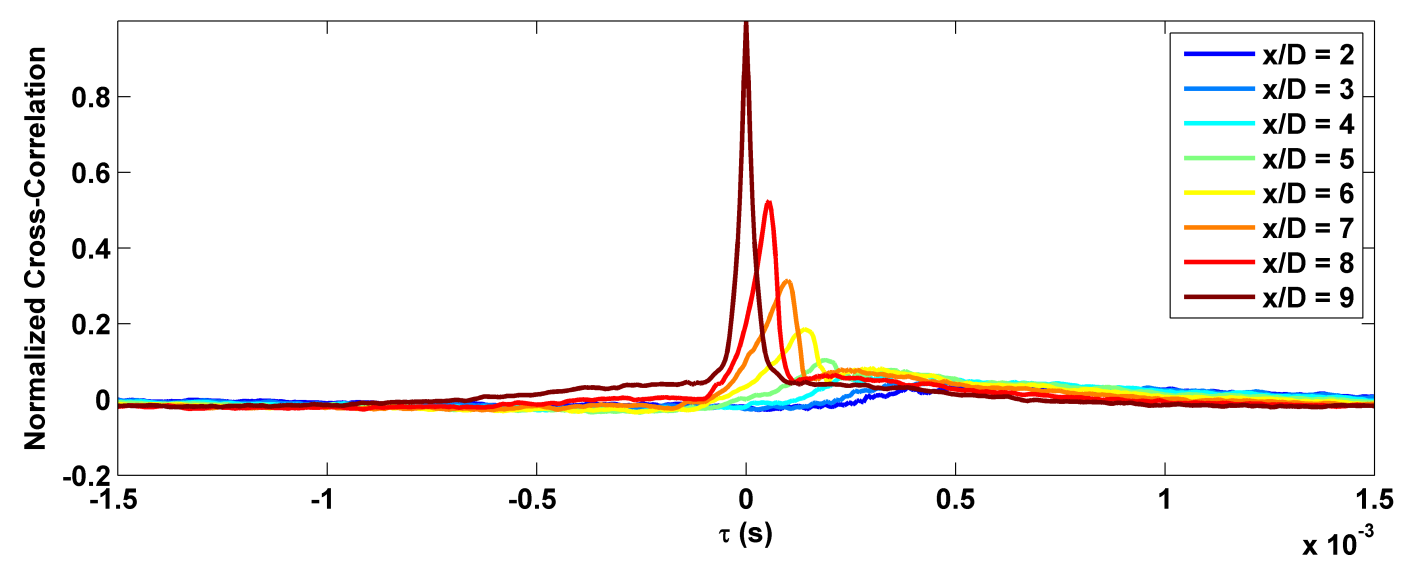

(b)

Figure 19. Normalized cross-correlations of the near-field pressure fluctuations for the forced jet with the reference microphone at $x / D=9$ : (a) First array location, (b) Last array location.

\subsubsection{Near Field to Far Field Cross-Correlations}

In Figure 20, the variation of the normalized cross-correlations of the near-field pressure along the first array position (Figure 20(a)) and last array position (Figure 20(b)) with the far-field microphone at $30^{\circ}$ are presented for the forced jet. It is evident from Figure 20 that the cross-correlations for both arrays presented are again similar to the 
unforced jet (Figure 12). The maximum correlation obtained in Figure 20(a) is approximately 0.17 (compared to 0.2 in the unforced jet). Furthermore, the evolution of the shape of the correlations with downstream displacement is analogous to the unforced jet. The reason for this similarity is again due to the low forcing Strouhal number used. The similarities can also be observed in Figure 21 which illustrates the maximum normalized cross-correlation to the $30^{\circ}$ far-field microphone for the forced jet. 


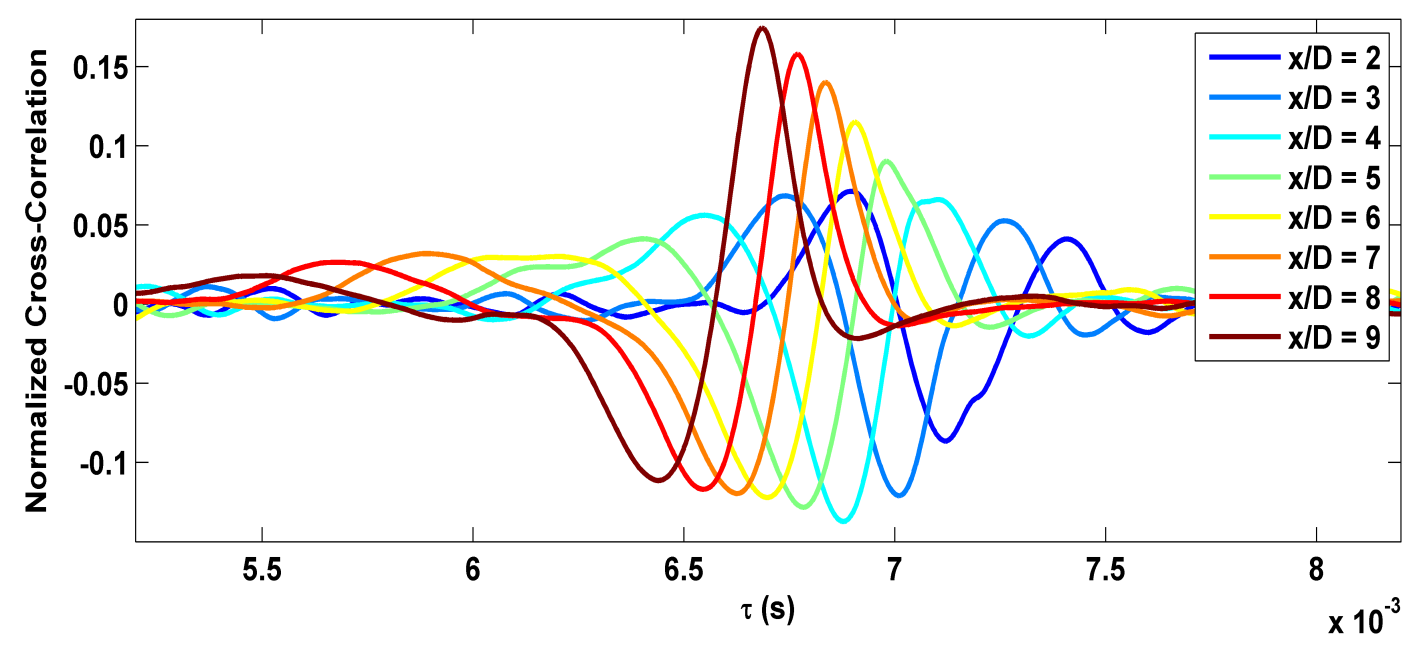

(a)

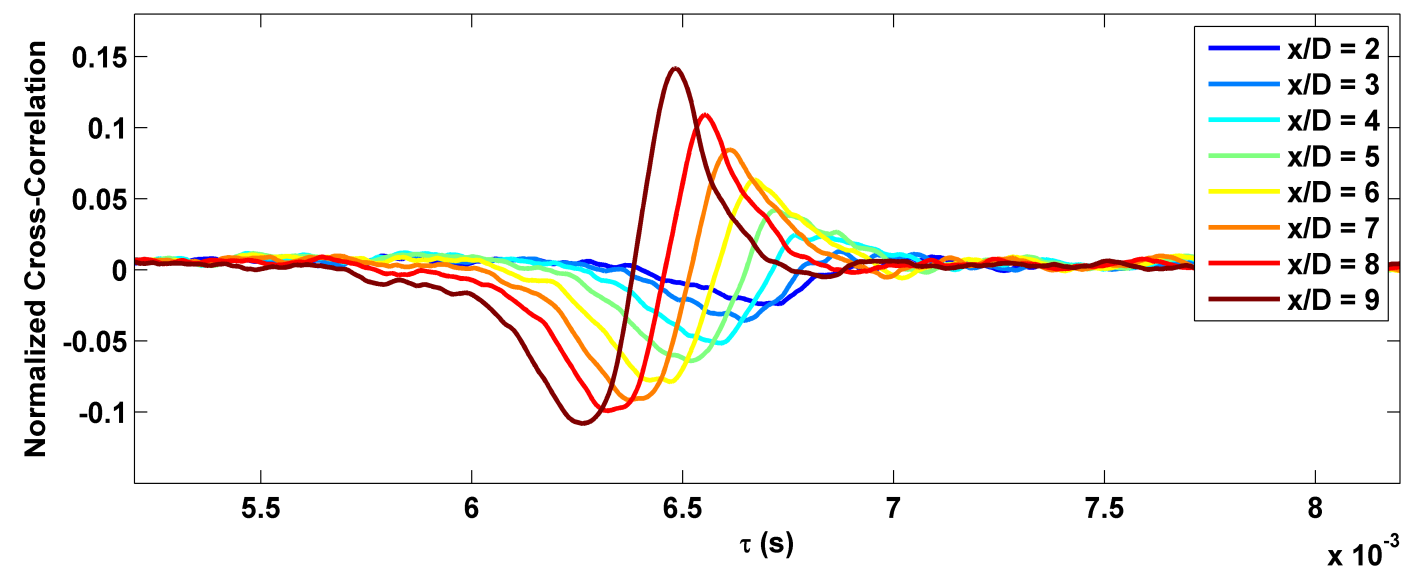

(b)

Figure 20. Normalized cross-correlation of the near-field pressure fluctuations for the forced jet with the $30^{\circ}$ far-field microphone: (a) First array location, (b) Last array location. 


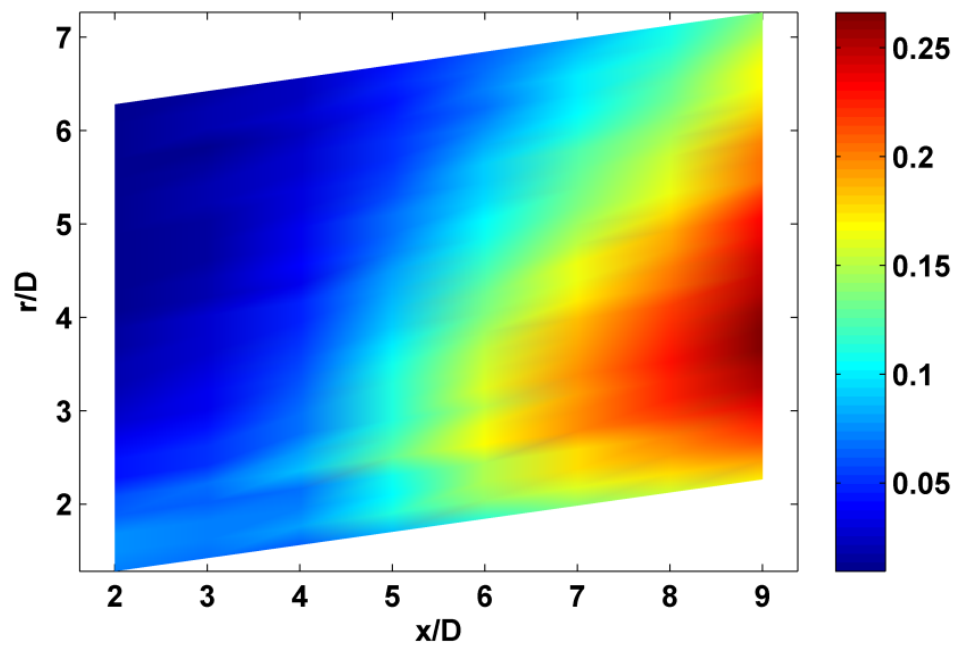

Figure 21. Maximum normalized cross-correlations of the near-field pressure fluctuations for the forced jet with the $30^{\circ}$ far-field microphone. 


\section{Chapter 6: Effects of Forcing: Phase-Averaging}

\subsection{Pressure Waveforms}

The main interest in the present analysis is the new information that the simultaneous acquisition of the actuation phase provides. The following sections describe the phaseaveraged pressure response at each microphone for forcing the axisymmetric mode at $S t_{D F}=0.02$ (corresponding to a frequency of approximately $250 \mathrm{~Hz}$ ).

\subsubsection{Near Field}

Figure 22 shows the phase-averaged pressure results for the first array position that is just outside of the shear layer. The most upstream microphone in this array position (Figure 22(a)) is located at $x / D=2$ and $r / D=1.28$. As can be seen in Figure 22(a), each actuation pulse generates two well-defined waves (in the case of the first microphone, the positive peaks of the two waves occur at phase angles of approximately $12^{\circ}$ and $29^{\circ}$ ), both with a positive excursion preceding a negative excursion. The first wave has smaller amplitude and arrives at the microphone much earlier. The triangular marker displayed in Figure 22(a) represents the expected time of arrival at the microphone of an acoustic wave travelling at the ambient speed of sound leaving the nozzle origin at zero phase. This expected time-of-arrival lines up well with the first compact wave in the upstream microphone, suggesting an acoustic phenomenon. Given these observed characteristics in addition to previous characterizations performed in Sinha et al. [28], this wave is understood to be the noise of the plasma breakdown travelling directly without being 
modified by the jet, i.e. actuator self-noise. The actuator self-noise dissipates quickly, becoming indistinct after the phase-averaging process at the downstream microphones (Figure 22(b)). The second compact wave generated by each actuation is much stronger and is representative of the hydrodynamic response of the jet (i.e. the signature of the large-scale structures) [28]. The leading positive excursion is the compressive front associated with the large-scale structure whose core manifests in the trailing negative excursion. Lastly, as expected the arrival of the large-scale structures seeded by the impulse occurs at later times for downstream microphones. Furthermore, the pressure fluctuations appear to dissipate with increasing axial distance as has been previously shown [28]. 


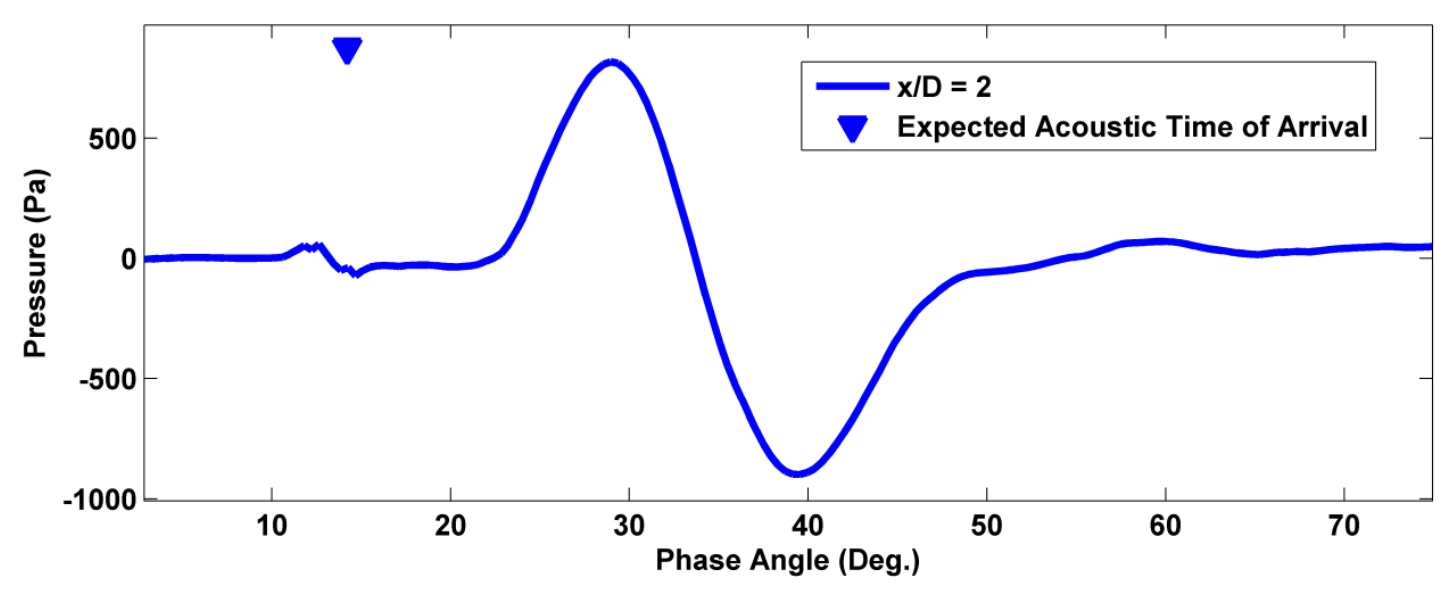

(a)

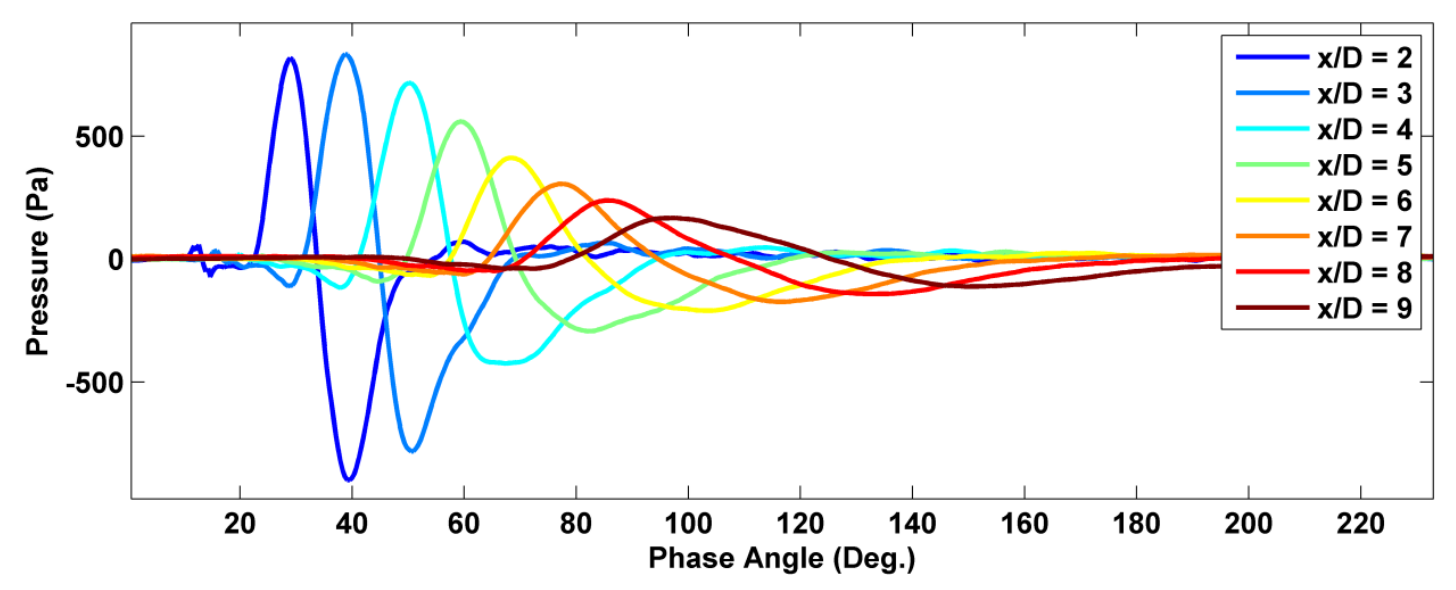

(b)

Figure 22. Phase-averaged waveform for $S t_{D F}=0.02$ : (a) $\mathrm{x} / \mathrm{D}=2, \mathrm{r} / \mathrm{D}=1.28$, (b) First array location for all microphones.

The phase-averaged waveform for the last radial position ( $\mathrm{z}$ in Figure $6(\mathrm{~b})$ ) is presented in Figure 23. Note over an order-of-magnitude scale change in the ordinates of Figure 22 and Figure 23. In this array position, the most upstream microphone is at $x / D=2$ and $r / D=6.28$ (Figure 23(a)). The expected time-of-arrival of an acoustic wave lines up fairly well with the first large amplitude wave over the entire axial extent 
indicating that this primary feature is actuator self-noise. Furthermore, this suggests that the array is no longer in the hydrodynamic field and thus does not capture the convective signature that is due to the large-scale structures in the shear layer. Unlike the first array location (Figure 22) where the second wave constitutes a positive excursion followed by a negative one, the latter array location constitutes mainly a positive excursion.

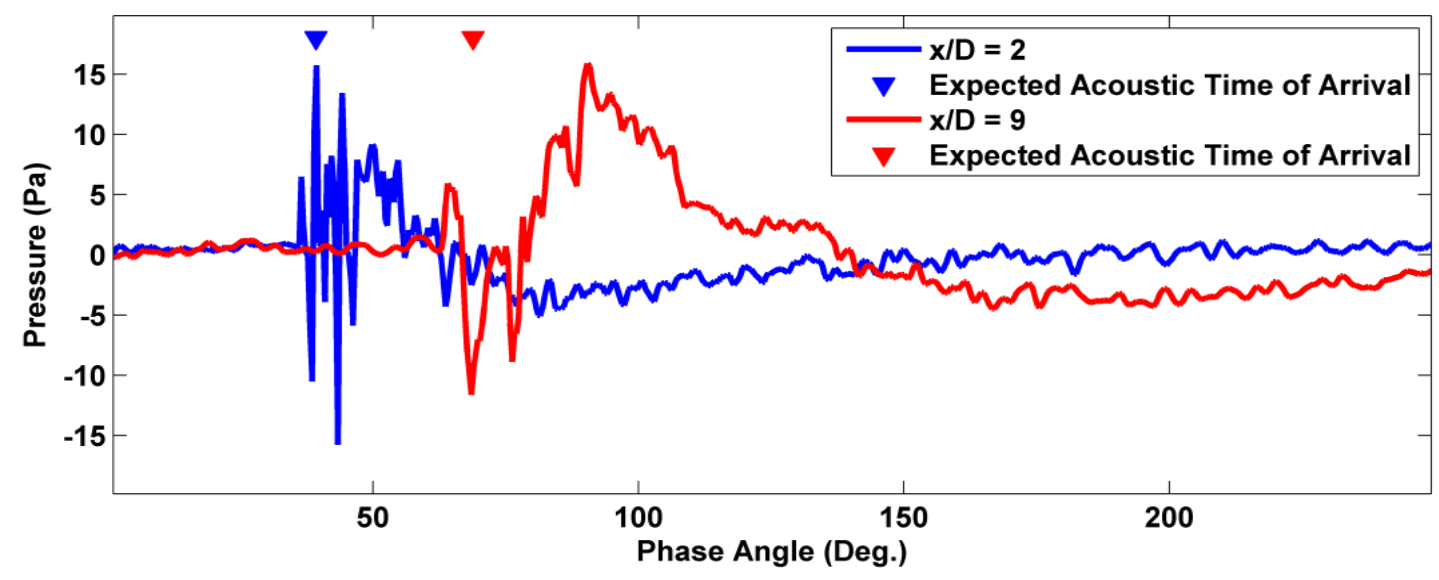

(a)

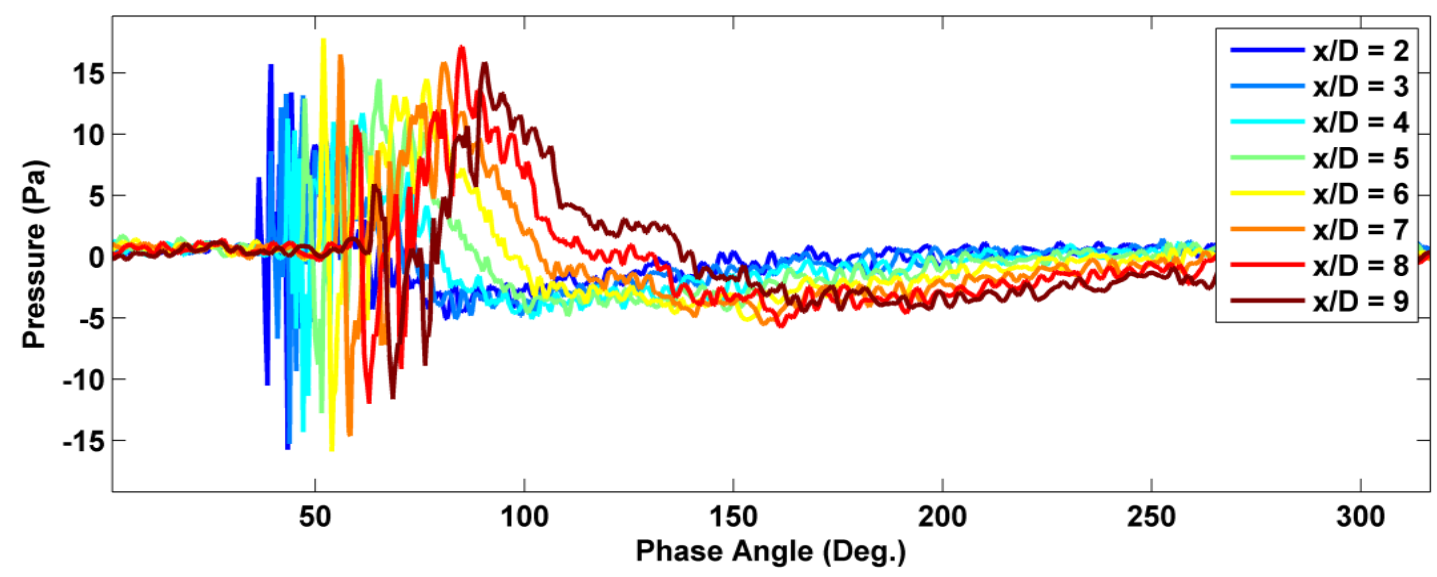

(b)

Figure 23. Phase-averaged waveform for $S t_{D F}=0.02$ : (a) $\mathrm{x} / \mathrm{D}=\mathbf{2}$ and 9 for the last array locations, (b) Last array location for all microphones. 


\subsubsection{Far Field}

The phase-averaged waveform for the $30^{\circ}$ far-field microphone is presented in Figure 24. As can be seen, the waveform is similar to that observed for the last microphone in the last array location (Figure 23(a)), with a negative excursion followed by a positive. Furthermore, the amplitude is small compared to the near-field microphones as expected. This again indicates that the convective signature that is due to the large-scale structures is not being captured at this location.

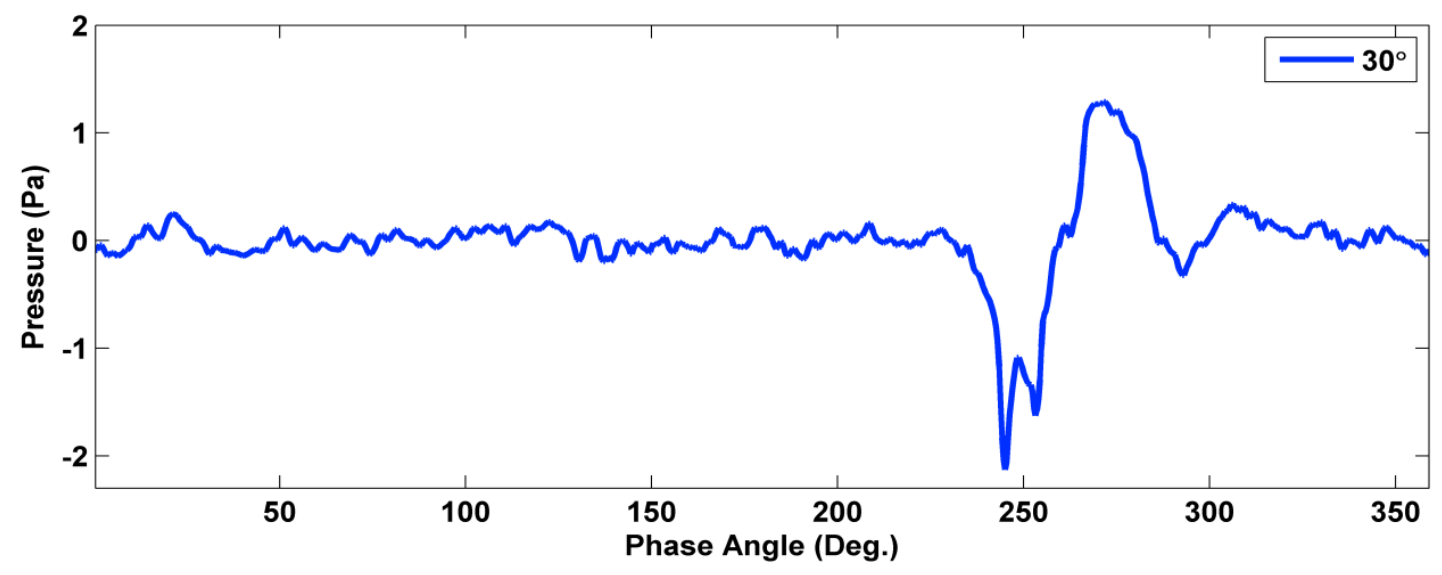

Figure 24. Phase-averaged waveform for the $30^{\circ}$ far-field microphone.

\subsection{Wavelet Filtering}

Figure 25 shows the phase-averaged waveforms at two radial positions $(r / D=1.28$ and $r / D=3.68)$ for the most upstream microphone. The expected time-of-arrival for an acoustic wave traveling directly from the actuators to the microphones as previously discussed is observed to align well with the first compact wave for the microphone at $r / D=1.28$ (Figure 25(a)) and with the first large amplitude wave for the position $r / D=3.68$ (Figure 25(b)). For radial positions close to the jet shear layer, as in Figure 
25(a), the near-field pressure signal is dominated by the hydrodynamic response of the jet. Here the actuator-self noise is producing an almost negligible amount of fluctuation, as was also shown in Figure 22. However, due to the relatively slow decay of the acoustic component in comparison to the more rapid decay in hydrodynamic fluctuations, as the microphone is traversed outwards, the actuator-self noise begins to represent a significant contribution to the overall intensity of the signal. Additionally, as in Figure 25(b), the arrival of the actuator self-noise begins to coincide with that of the signature of the largescale structures obscuring the near-field response and making analysis techniques, such as cross-correlations, difficult to interpret. Even though the speeds of the two signals are quite different, this occurs because of the significant differences between the distances the two signals must travel to get to the microphone. Therefore, it is necessary to apply a filter to the phase-averaged waveforms to remove the actuator self-noise. 


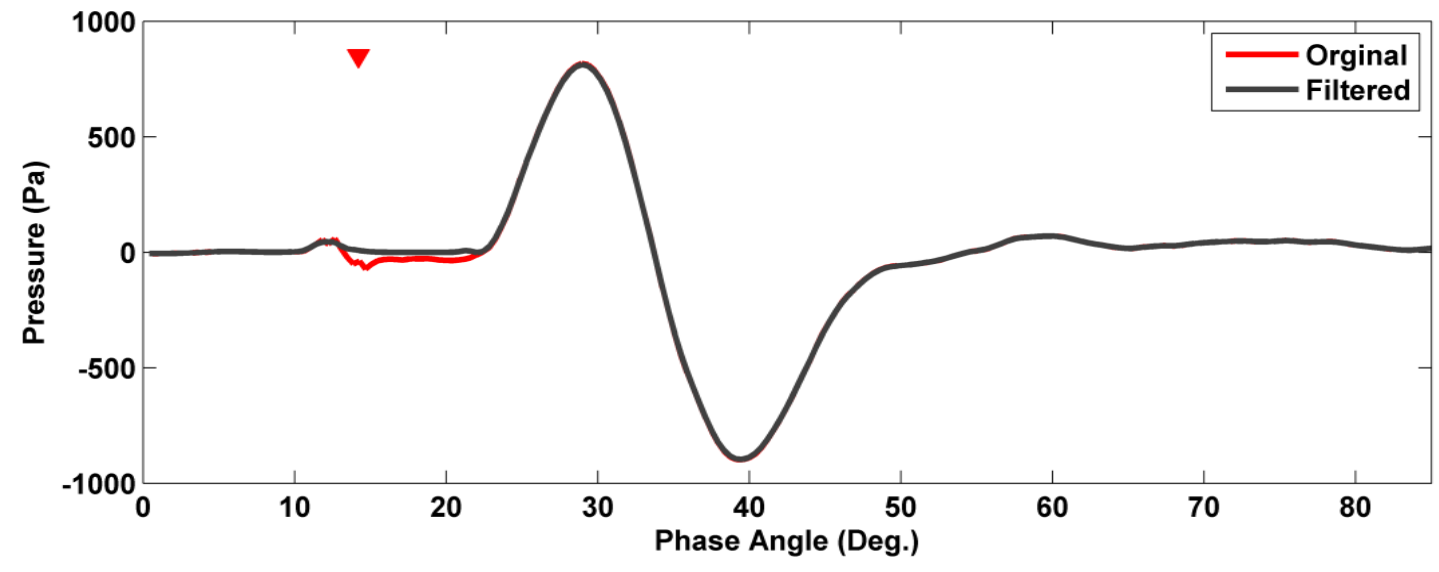

(a)

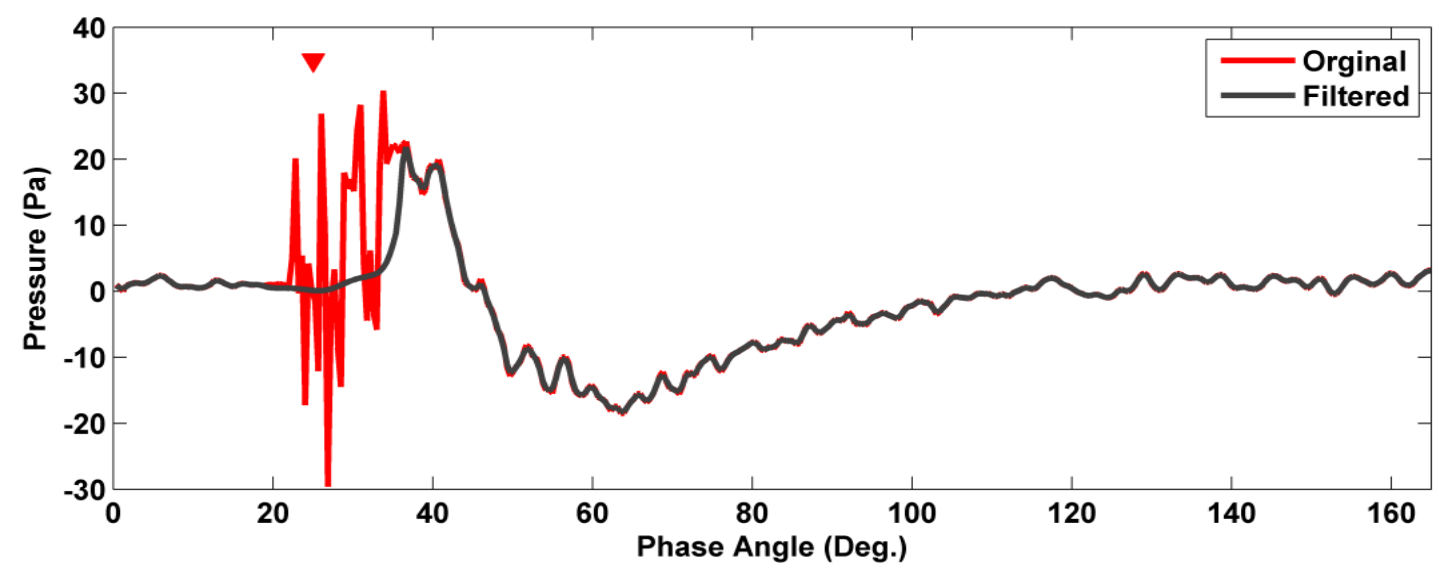

(b)

Figure 25. Phase-averaged waveforms for original and filtered waveforms: (a) $x / D=2.0, r / D=1.28$, (b) $x / D=2, r / D=3.68$.

The filtering algorithm consists of a continuous wavelet transform applied to both the phase-averaged response and the instantaneous pressure signals presented in Chapter 5. The $4^{\text {th }}$-order Paul wavelet is one of the most suitable wavelets available among the numerous mother wavelets. The similarity of the shape of the imaginary part of this mother wavelet, even though the signal is inverted, with the phase-averaged response is 
particularly notable as can be seen in Figure 26 . In the wavelet domain the actuator selfnoise produces high frequency oscillations, which can be filtered out via a smoothing operation (i.e. moving average). After smoothing in the wavelet domain, the waveform is transformed back into the temporal domain where it then undergoes another smoothing operation in order to remove any small oscillations that were introduced into the signal during the reconstruction. As can be seen in Figure 25, the near-field hydrodynamic signal is unaffected by the filtered algorithm, whereas the actuator self-noise has been almost entirely removed. Unless otherwise mentioned, the wavelet filter will be applied to all pressure signals for the forced jet.

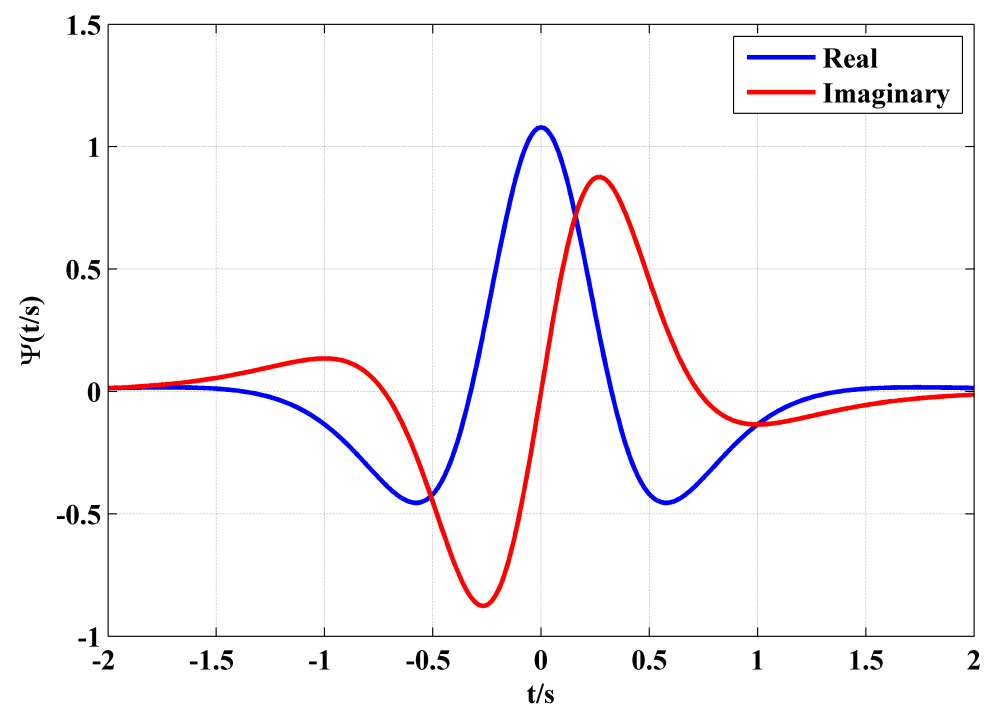

Figure 26. $4^{\text {th }}$ Order Paul wavelet sample.

\subsection{Correlations}

\subsubsection{Autocorrelations}

The autocorrelation functions of the phase-averaged pressure signals are presented in Figure 27 for $x / D=6$ and $x / D=9$. It can be seen that the autocorrelations of the phaseaveraged waveforms differ from those of the instantaneous forced signals (Figure 17) and 56 
the unforced jet (Figure 10). The presence of the deep negative peaks present for the first few array locations is similar to those observed for the other two cases, as well as the broadening of the half-widths with downstream streamwise distance. However, the main difference is that as the microphones are placed radially away from the jet shear layer, the half-widths of the autocorrelations become broader which is the opposite of what has been previously observed in the instantaneous forced signals and the unforced jet. This comparison can be better observed in Figure 28 where the half-width of the autocorrelations for the last microphone on the array $(x / D=9)$ at the first and last array locations are presented. For the first array location (Figure 28(a)), the autocorrelation of the phase-averaged signal is significantly broader than the other two functions, but still follows the trend closely. However, it can be observed in Figure 28(b) that when the microphone is placed away from the shear layer, the half-width of the autocorrelation is even further broader than both other functions presented and it is also broader than the first array location. These observations require further inquiry as they are unexpected. 


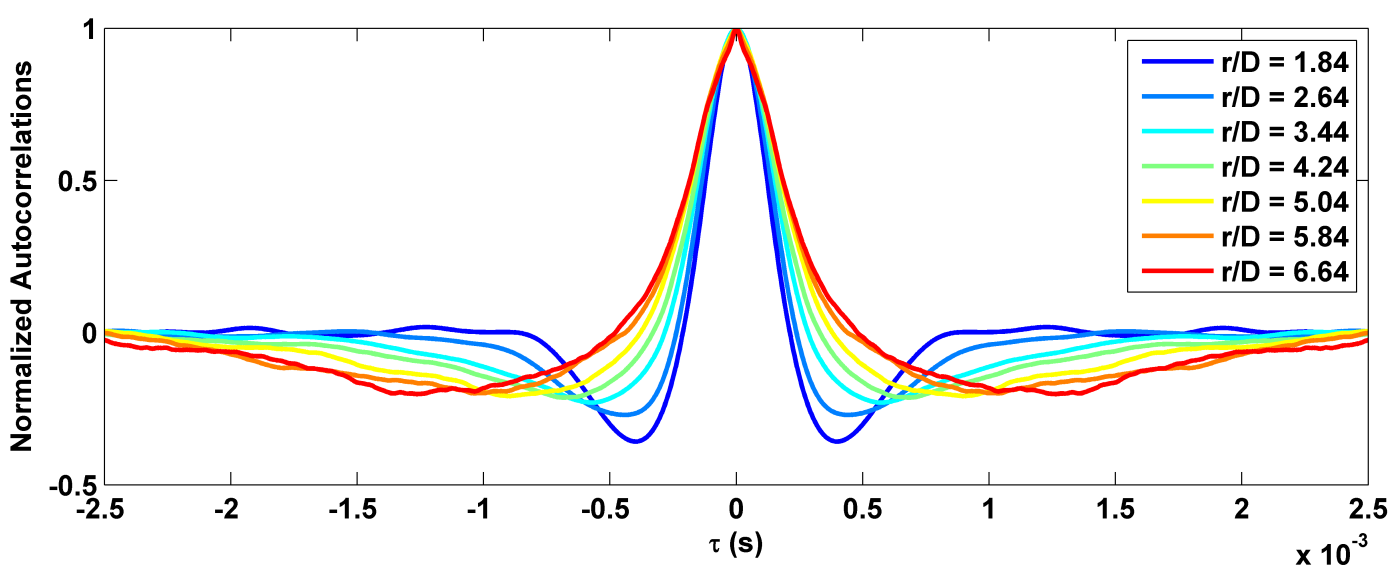

(a)

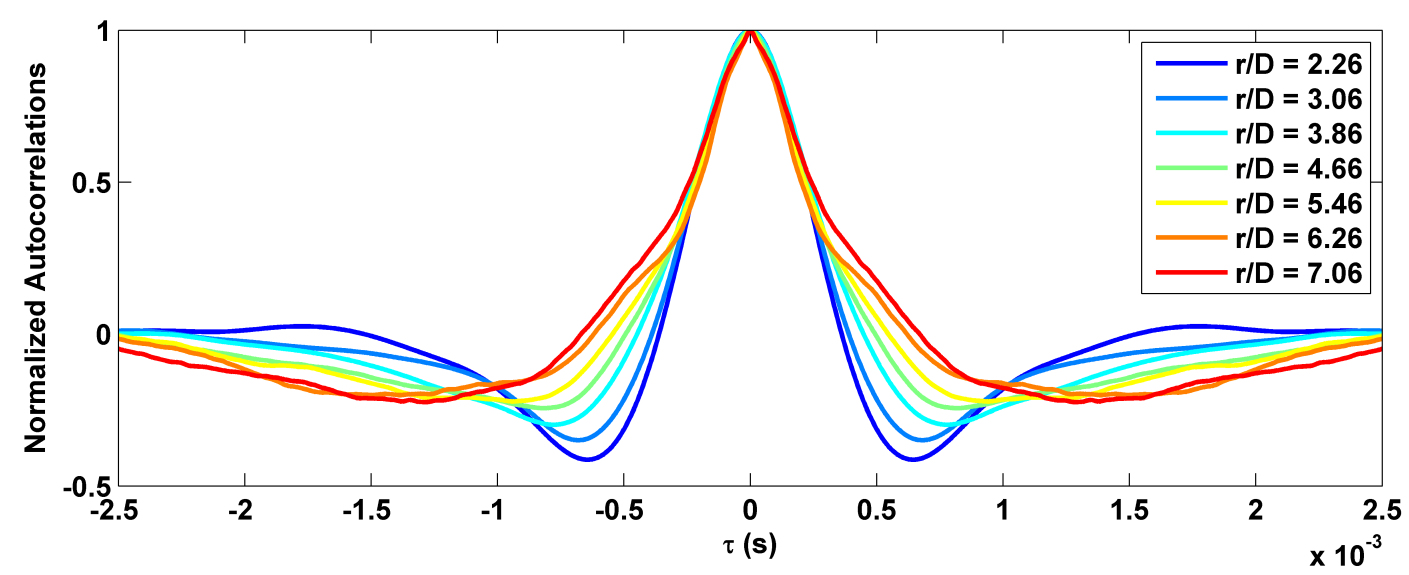

(b)

Figure 27. Normalized autocorrelations of the phase-averaged waveforms at the same axial position and various radial locations: (a) $x / D=6$, (b) $x / D=9$. 


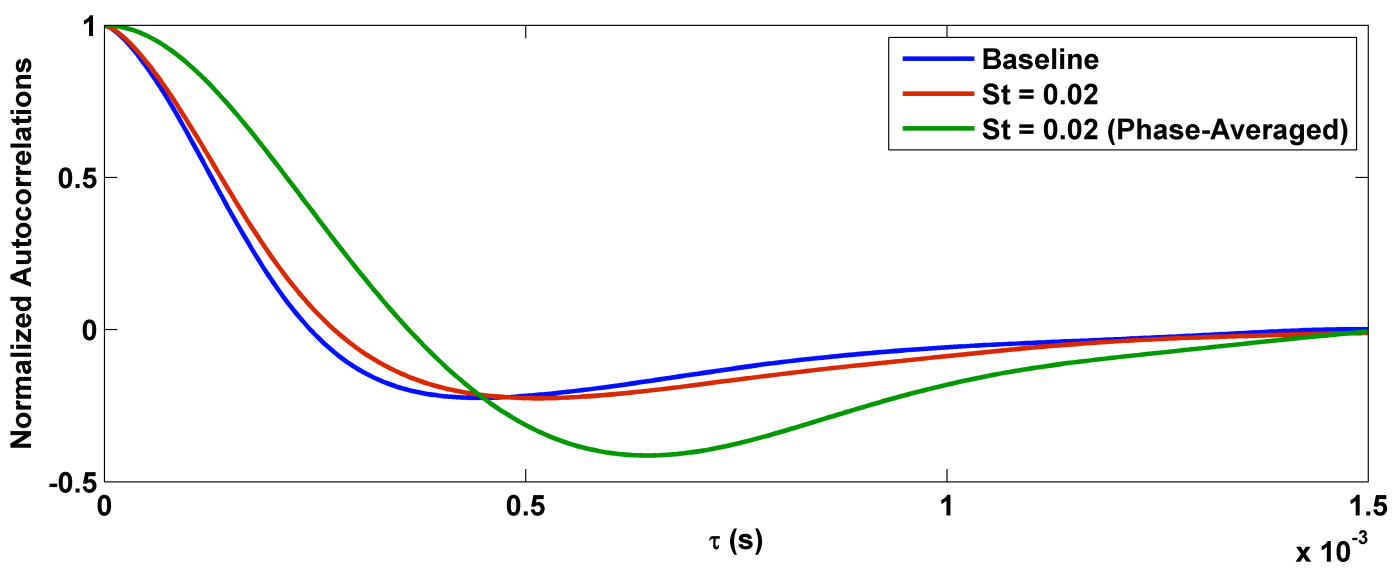

(a)

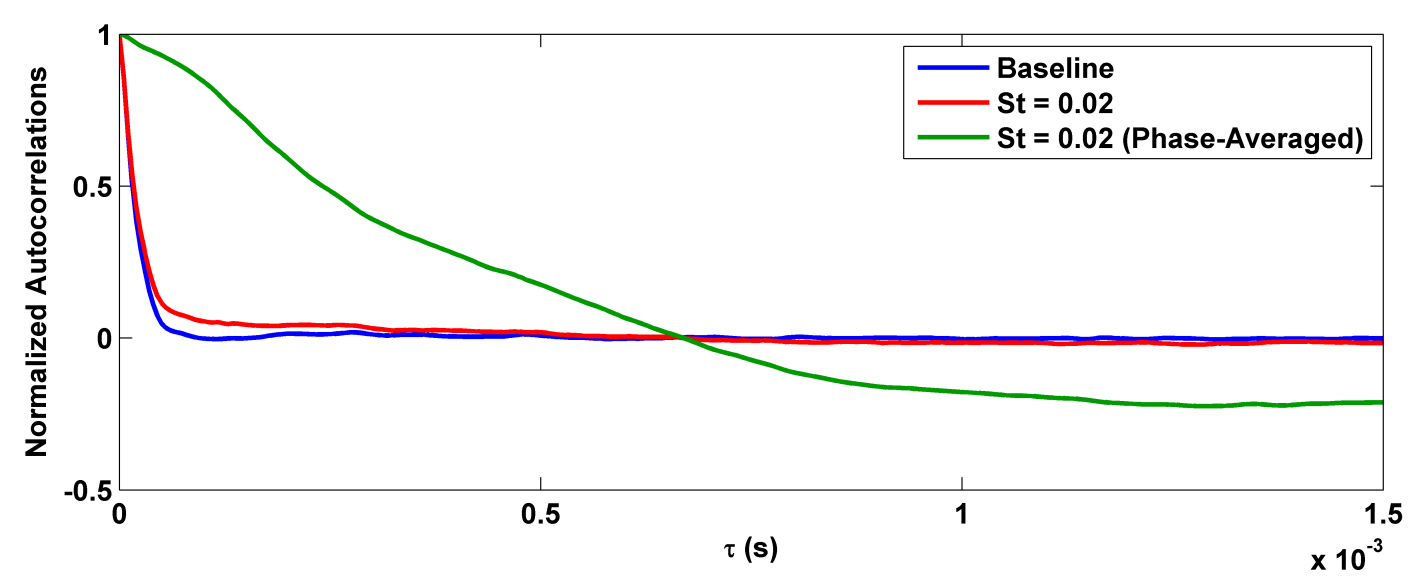

(b)

Figure 28. Normalized autocorrelations of the phase-averaged waveforms at $x / D=9$ : (a) First array location, (b) Last array location.

\subsubsection{Near Field Cross-Correlations}

The normalized cross-correlations of the phase-averaged waveform for the forced jet with the reference microphone at $x / D=9$ (similar to Figure 11) are presented in Figure 29. Once again, correlation is noticeable over the entire axial extent for the forced jet, even with quite large distances. Furthermore, as the probe is placed closer to the 
reference microphone the shape of the correlation resembles that of the autocorrelation, as is expected. It is interesting to note that as $\Delta x<4 D$, a second negative excursion following the positive is observed similar to the autocorrelation. However, this is not the case in the instantaneous forced jet (Figure 19(a)) where the cross-correlations consist of simply a negative excursion preceding a positive. Lastly, the correlation shape of the last array position (Figure 29(b)) is initially roughly symmetric with the negative excursion rapidly decreasing as the jet evolves downstream. This is dissimilar to the unforced jet (Figure 11(b)) and the instantaneous forced signals (Figure 19(b)) where no negative excursions are observed and the widths of the positive peaks are very narrow. 


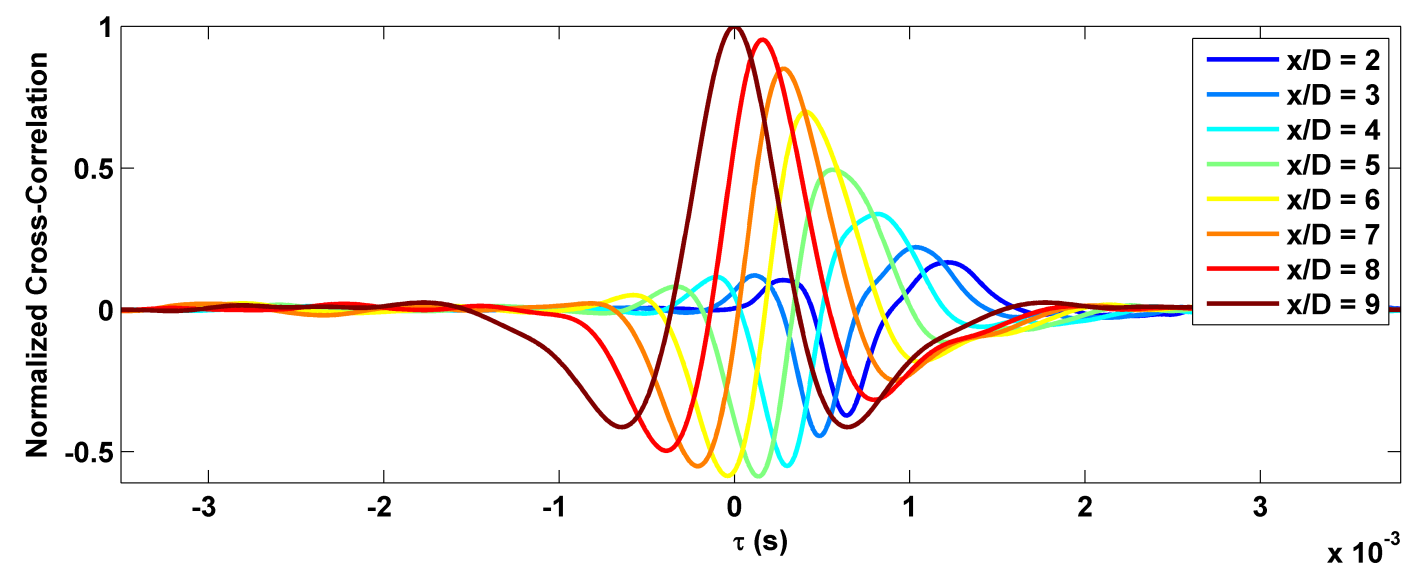

(a)

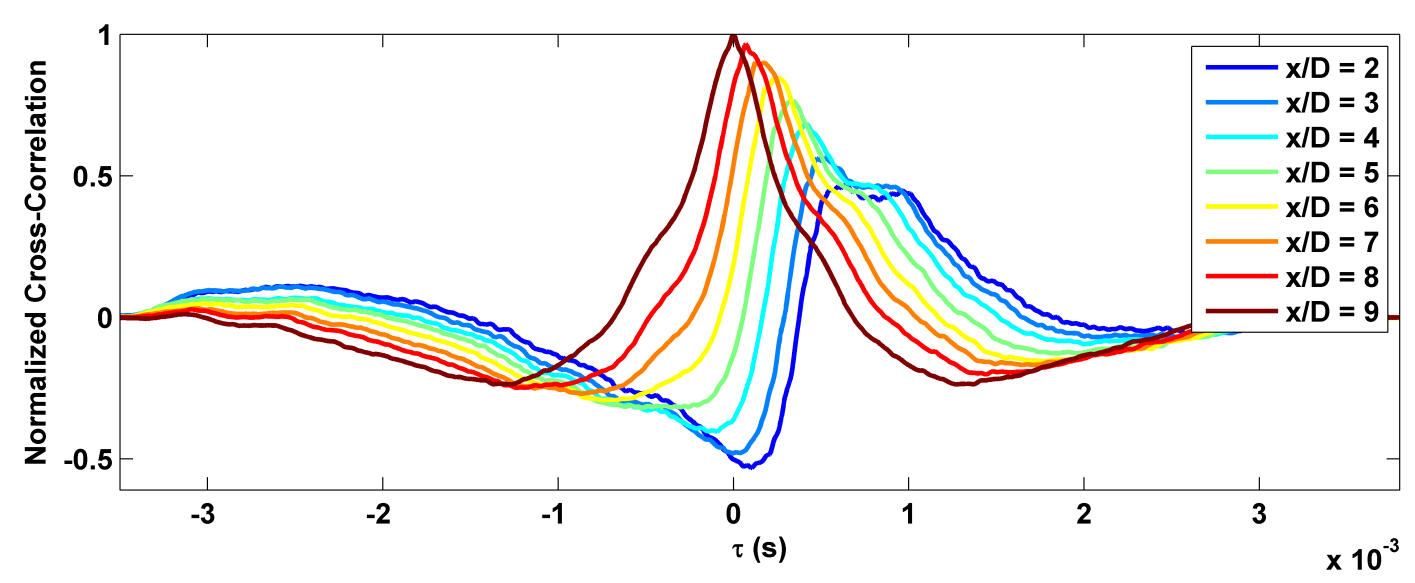

(b)

Figure 29. Normalized cross-correlations of the phase-averaged waveforms with the reference microphone at $x / D=9$ : (a) First array location, (b) Last array

\subsubsection{Near Field to Far Field Cross-Correlations}

\subsubsection{Individual Array Locations}

The downstream variation of the normalized cross-correlations of the phase-averaged near-field and far-field signals along the first array position and last array position with the far-field microphone at $30^{\circ}$ are shown in Figure 30(a) and (b), respectively. It is 
apparent from Figure 30 that the negative peaks are more dominant in both array sets. This differs from the correlations involving the instantaneous signals in the forced jet (Figure 20) as well as the unforced jet (Figure 12), where the positive peaks exhibited higher amplitude. As illustrated in Figure 30(a), the correlation increases as the jet evolves downstream, reaching a maximum of approximately 0.5 (compared to roughly 0.17 in the instantaneous forced case) between $x / D=5$ and 8 , at which point the correlation decreases slightly. The maximum correlation has also shifted upstream in comparison to the forced instantaneous case and the unforced jet. This observation corresponds with the shift seen in Figure 16(b). The downstream evolution of the correlation in Figure 30(b) is similar to that seen in Figure 29(b). Similar to the correlations involving the instantaneous signals in the forced jet, the correlation increases as the jet evolves downstream; however, this sustained growth is only visible for the positive excursions. 


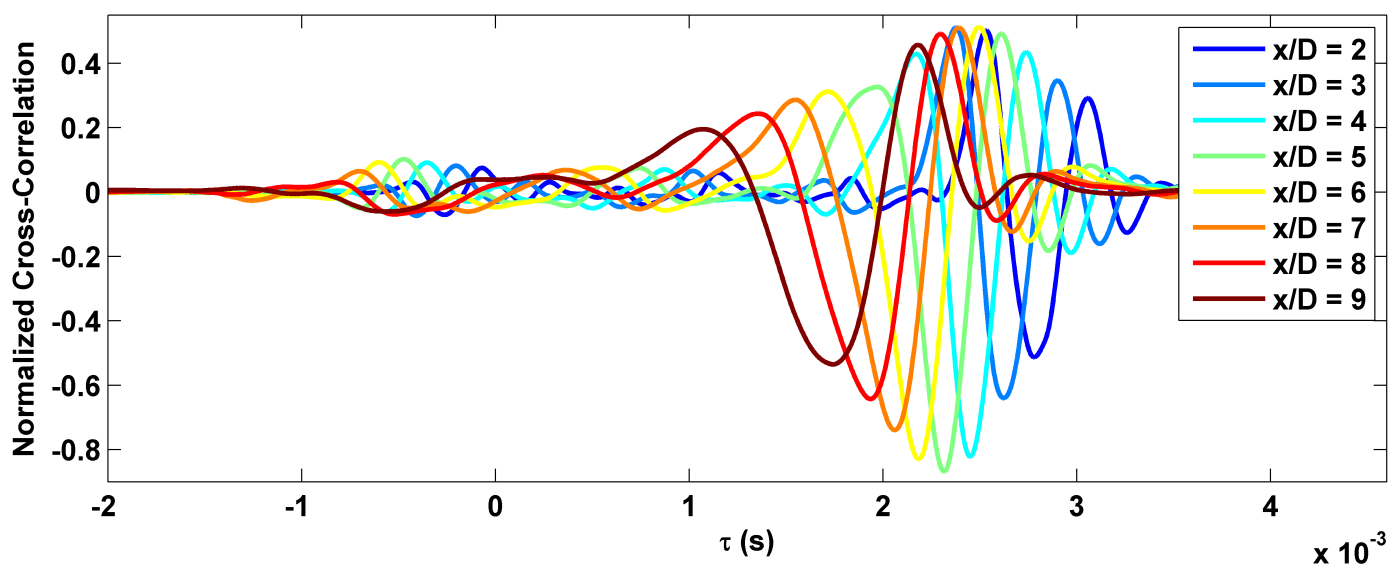

(a)

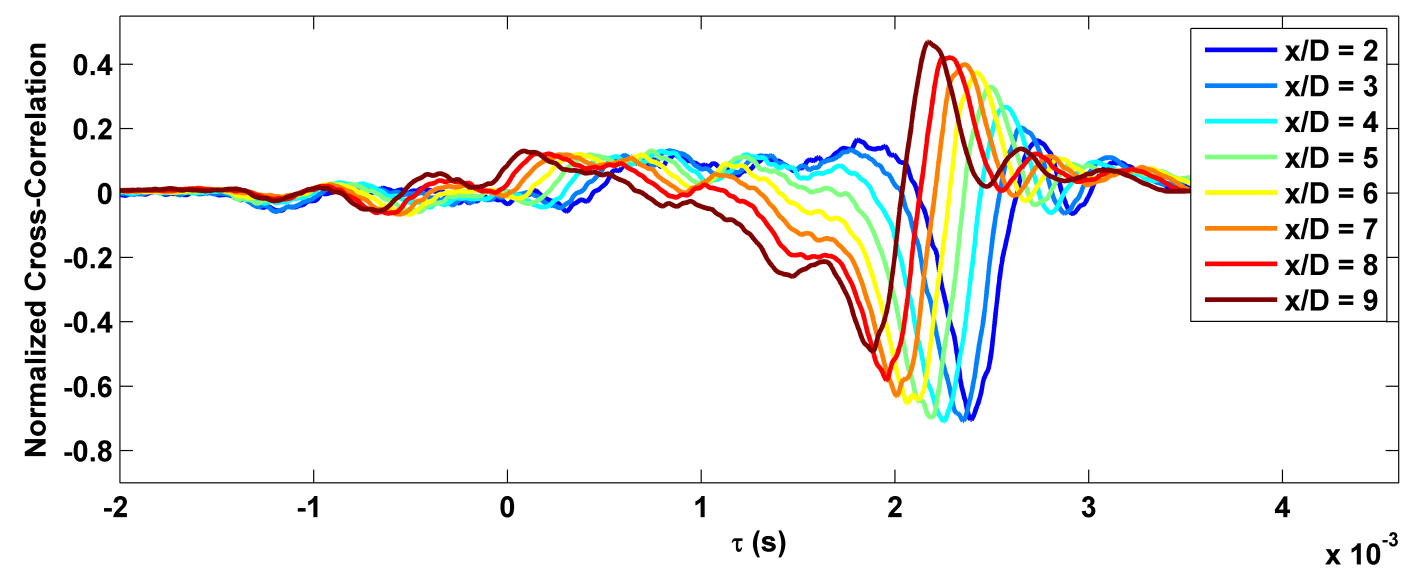

(b)

Figure 30. Normalized cross-correlations of the phase-averaged waveforms with the $30^{\circ}$ far-field microphone: (a) First array location, (b) Last array location.

\subsubsection{Maximum Correlation Map}

Figure 31 shows the maximum normalized cross-correlation to the $30^{\circ}$ far field for each microphone location, computed for the phase-averaged waveforms. Several differences between Figure 31 and the instantaneously forced jet (Figure 21) are evident. The maximum correlation values for the phase-averaged signals are significantly 
increased $(\sim 0.6$ versus $\sim 0.3)$ over the instantaneous forced signals. The maximum correlation values have also shifted to noticeably higher angles (the angles are measured similar to Figure 14) for the phase-averaged waveform signals. The upstream, far radial locations still have low correlation to the far field at $30^{\circ}$ (relative to the rest of the microphone positions). However, for the region close to the nozzle exit $(x / D<3$ and $r / D$ $<2$ ), the correlation values are observed to be unusually high in relation to the surrounding areas. In this upstream region, the cross-correlation function is correlating primarily to hydrodynamic fluctuations, which can be seen with the rapid decay of the maximum correlation values with radial distance. On the other hand, in the downstream region, the correlation function is correlating primarily to acoustic fluctuations hence the decay in the maximum correlation values with polar angle.

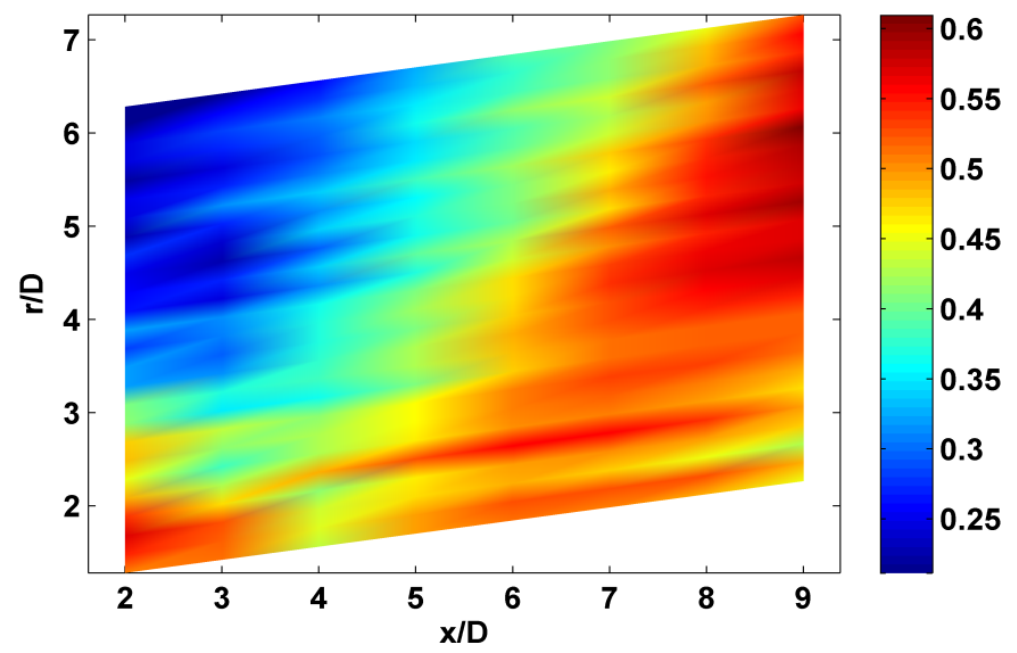

Figure 31. Maximum normalized cross-correlations of the phase-averaged waveforms with the $30^{\circ}$ far-field microphone. 


\subsection{Forcing Frequency Effects}

The effects of the forcing Strouhal number on the jet response are briefly discussed in this section. It was previously shown by Sinha et al. [28] that the fundamental response of the jet is seen to remain unchanged up to $S t_{D F}=0.11$. The periodicity only results in more pulses being captured within the averaging window. Increasing the $S t_{D F}$ above this value results in an almost sinusoidal response. Using linear superposition, it was shown that forcing at a higher $S t_{D F}$ results in a quasi-linear interaction between the seeded large-scale structures and the succeeding structures in the wave train. In this section, a forcing Strouhal number of 0.15 is used to briefly investigate the effects that the interaction has on the phase-averaged signals, as well as the autocorrelations.

\subsubsection{Phase-Averaged Waveforms}

Figure 32 shows the phase-averaged pressure results for the first and last array positions. Note that the wavelet filtering has been applied to the pressure waveforms presented. As can be seen in Figure 32(a) for the first array location, the pressure waveforms are more of a sinusoidal response. As the waveform is traversed downstream, it becomes broader and the amplitude decreases. This is due to the interaction between the seeded structures with the succeeding structures in the wave train. For the last array location (Figure 32(b)), the amplitudes of the pressure waveforms are reduced significantly similar to what was observed for the lower forcing Strouhal number (Figure 23(b)). For this array, the upstream waveforms have small magnitudes, while the downstream microphones constitute a small negative excursion followed by a positive. 


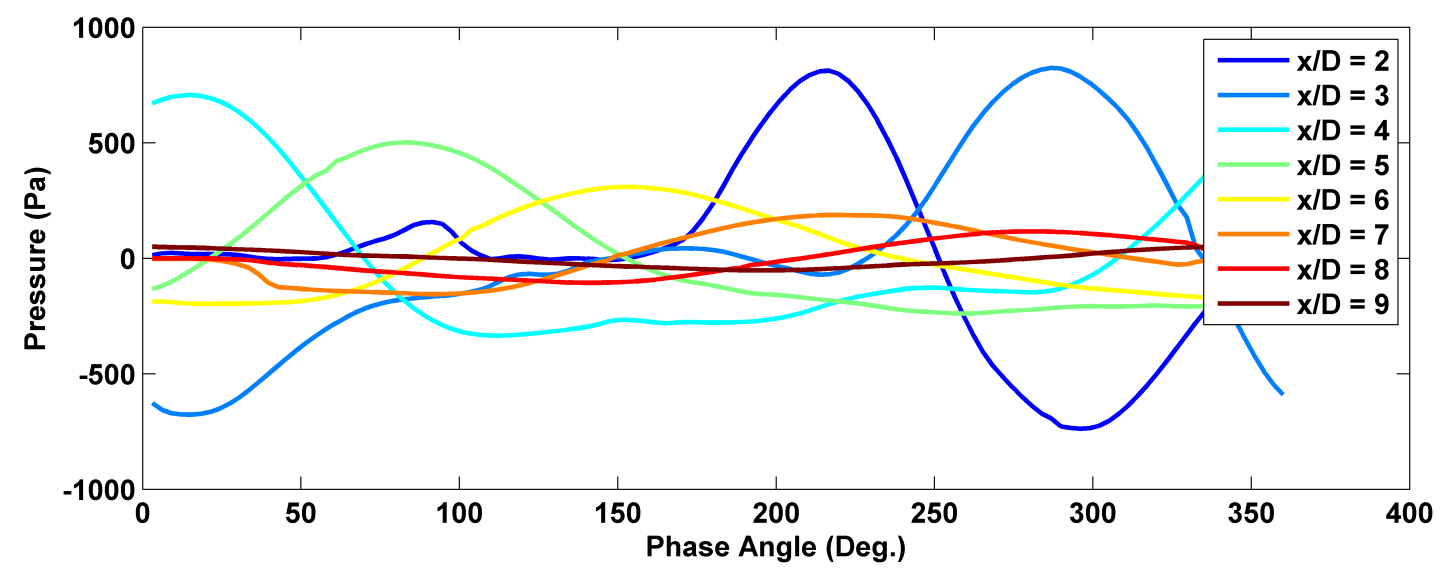

(a)

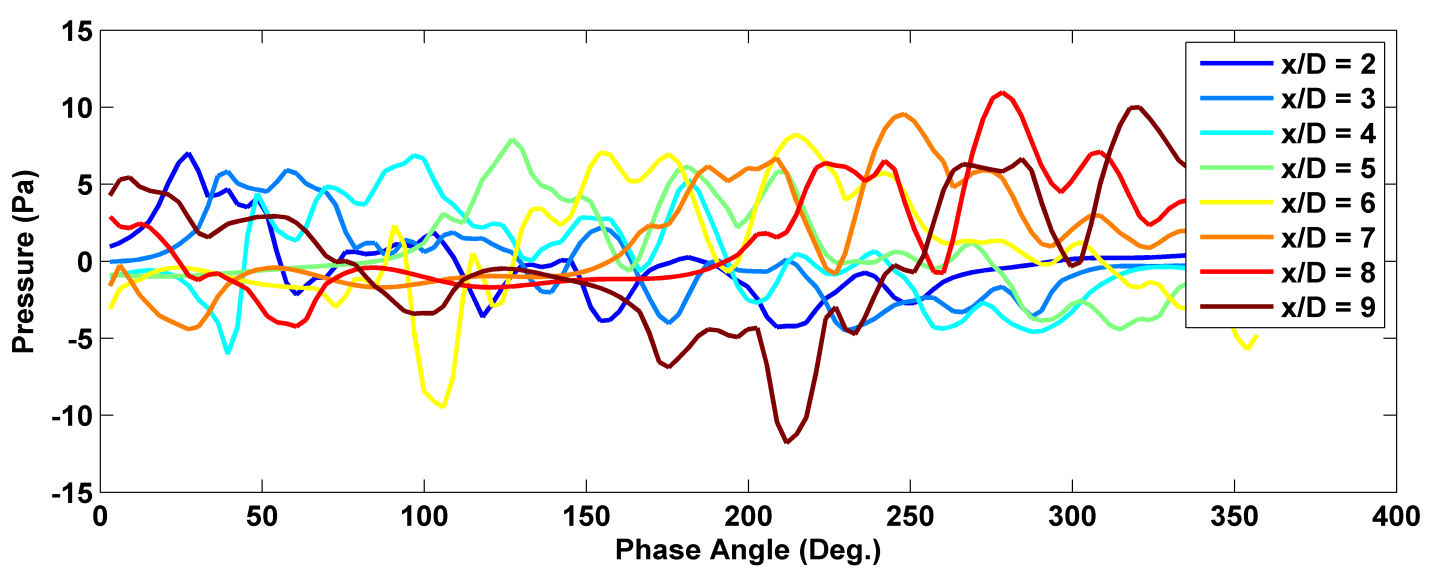

(b)

Figure 32. Phase-averaged waveform for $S t_{D F}=0.15$ : (a) First array location, (b) Last array location.

\subsubsection{Autocorrelations}

The normalized autocorrelations of the instantaneous near-field pressure and the phase-averaged waveforms at $x / D=9$ for various radial locations are presented in Figure 33. Immediately, it is evident that the trend observed for the autocorrelations of the instantaneous signals (Figure 33(a)) for the higher $S t_{D F}$ are similar to both the lower $S t_{D F}$ 
(Figure 17(b)) and the unforced jet (Figure 10(b)). The half-widths of the autocorrelation functions become narrower as you move away from the jet shear layer. For the phaseaveraged signals (Figure 33(b)), however, the half-widths do not vary is size with radial distance. Furthermore, deep negative peaks occur for all array locations, which is dissimilar to the lower $S t_{D F}$ (Figure 27(b)). A closer comparison between all signals discussed in this study is provided in Figure 34 for the last microphone in the array at both the first and last array location. In both array locations, the instantaneous near-field pressure signals are analogous to the unforced jet. The phase-averaged higher $S t_{D F}$ exhibits a deeper negative peak than the other autocorrelation functions, which could be a result of the close-by distance and interaction of the large-scale structures at this higher $S t_{D F}$. Furthermore, the lower $S t_{D F}$ displays a broader half-width than the higher $S t_{D F}$. This is a result of the forcing Strouhal number, a lower Strouhal number would result in a structure with a longer spatial and time extend hence the broader half-width observed. Again, further inquiry is required to better understand the trends observed for the phaseaveraged results. 


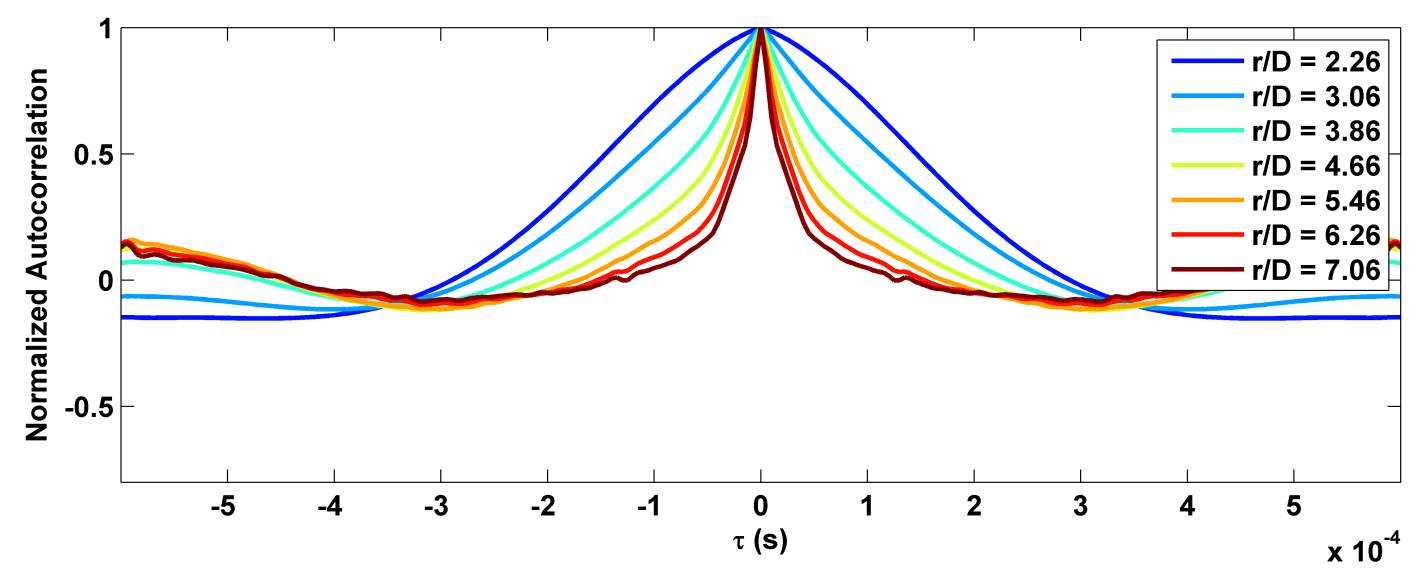

(a)

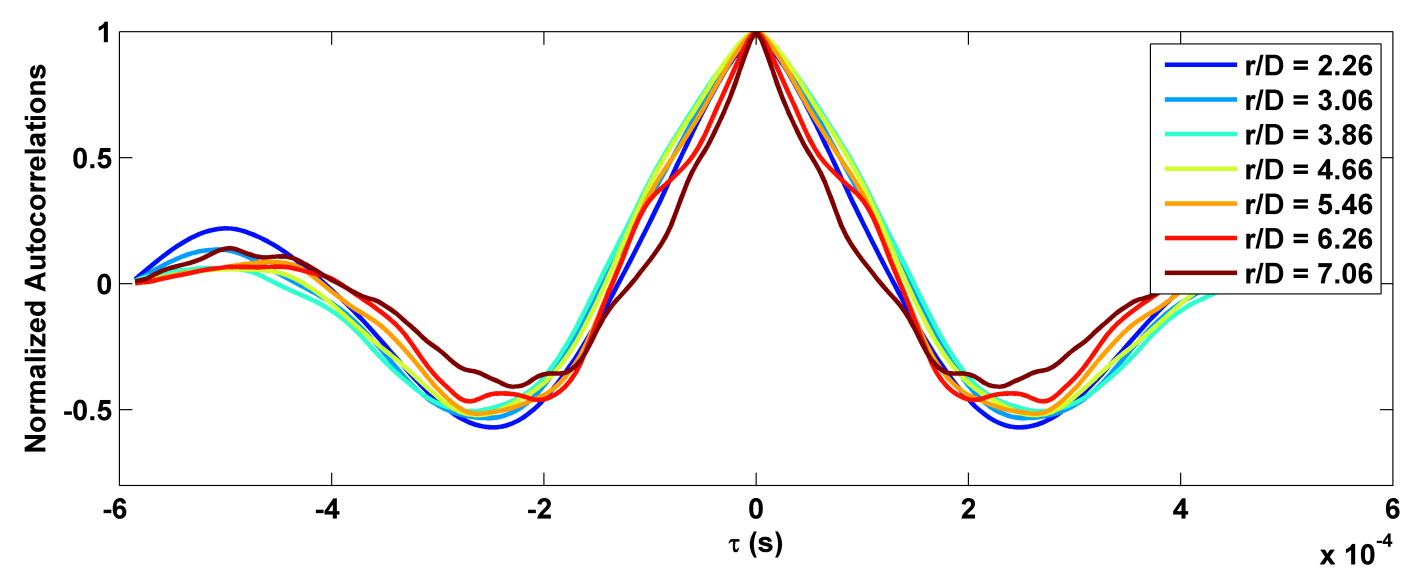

(b)

Figure 33. Normalized autocorrelations of the forced jet at $S t_{D F}=0.15$ at $x / D=9$ for various radial locations: (a) Instantaneous near-field pressure, (b) Phaseaveraged waveform. 


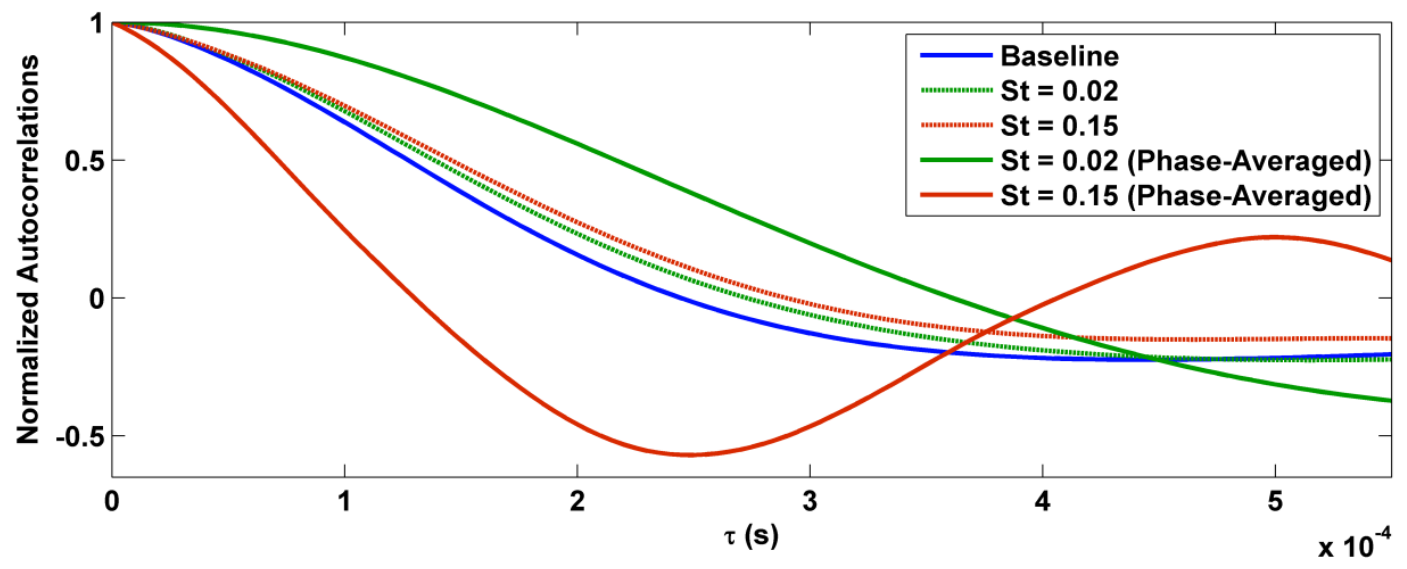

(a)

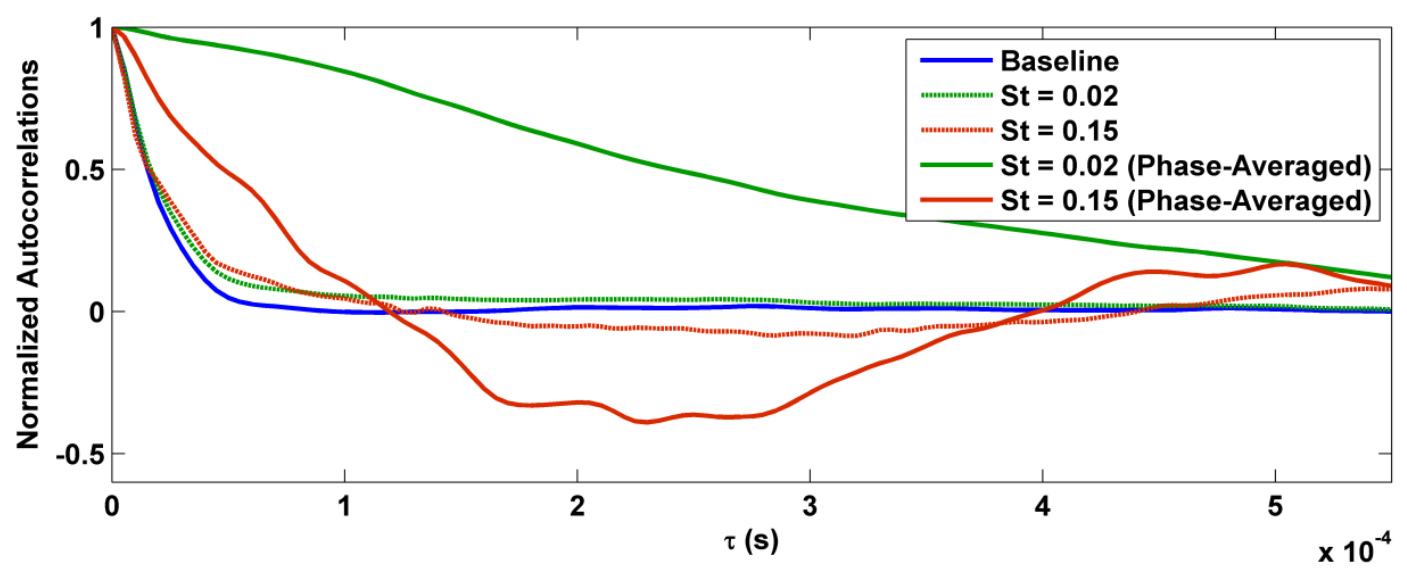

(b)

Figure 34. Normalized autocorrelations at $x / D=9$ : (a) First array location, (b) Last array location. 


\section{Chapter 7: Conclusions}

The response of a high-speed subsonic jet to low-frequency forcing was investigated using localized arc filament plasma actuators (LAFPAs). These actuators have demonstrated strong control authority on high-speed turbulent jets and provide a unique opportunity to study the dynamics of large-scale structures. Eight LAFPAs uniformly arranged around the periphery of the nozzle exit are operated in phase to simulate axisymmetric forcing. The jet Mach number and Reynolds number based on the jet diameter were 0.9 and $6.2 \times 10^{5}$. A traversing linear array which spans eight nozzle exit diameters in the axial direction and five nozzle exit diameters in the radial direction is employed in order to obtain a comprehensive picture of the near hydrodynamic and acoustic fields.

Phase averaging of the pressure signals with respect to the actuator signal was used to separate the relevant features of the response from the background turbulence. The phaseaveraged pressure signal of the jet forced at a Strouhal number of 0.02 exhibited a compact sinusoidal wave with a positive excursion preceding a negative excursion; this was demonstrated to be a hydrodynamic response and travelling with the convective speed of the flow structures in the streamwise direction. These hydrodynamic fluctuations were shown to decay rapidly with radial distance. A second compact wave in the phaseaveraged pressure waveform, which is of smaller amplitude (in the first array location) and decreased with downstream distance from the nozzle, was shown to be the actuator 
self-noise traveling with the ambient speed of sound. As the traverse moved radially away from the nozzle, the actuator-self noise became a significant contribution to the overall intensity of the near-field signal. Furthermore, the phase-averaged pressure at the last array location (which is five nozzle exit diameters away from the jet shear layer) mainly constituted a positive excursion. Owing to the growth in amplitude of the actuator noise in the latter array locations, the phase-averaged pressure signal was filtered utilizing a continuous wavelet transform in order to avoid making subsequent analyses difficult to interpret.

Simultaneous measurements of acoustic far field were also performed along with the near field, allowing the relationship between the near-field pressure and the far-field acoustics to be examined. Although the near-field pressure near the jet shear-layer was found to be largely hydrodynamic in nature, there was still significant correlation between that pressure and the far-field acoustic ( $30^{\circ}$ far-field microphone), particularly in the region past the end of the potential core. However, this region shifted upstream when the jet was forced at a Strouhal number of 0.02. It is important to note that this trend was observed for the correlations on the phase-averaged waveforms and do not offer a direct comparison with the unforced jet. Autocorrelation functions of the unforced jet further illustrated the transition of the signal from hydrodynamic in nature to acoustic. For array locations near the jet shear layer, the shape of the autocorrelation function was typical of that of a pressure wave with a negative excursion, however, as the radial distance increases the shape of the autocorrelation became typical of that of an acoustic signature. Forcing the jet at a low Strouhal number resulted in as similar autocorrelation function to 
the unforced jet. This was as a result of the low Strouhal number used and therefore the sparse presence of the new structures due to forcing in the flow. A similar trend was observed for the higher Strouhal number despite the fact that at this forcing frequency the structures are close together and interacting. When phase-averaging the signals, the autocorrelation functions were intriguing. For both Srouhal numbers used, the autocorrelations of the phase-averaged signals appeared to be dominated by the pressure field even at the last array location, which has been shown to consist of acoustic fluctuations.

The work presented in this thesis is ongoing and currently underway to further examine and understand the trends observed in the correlations by comparing the experimental results to the large-eddy simulation (LES) to better understand what is occurring within the jet. The present analysis can also be further examined by looking at various decomposition techniques that would allow the separation of the acoustic and hydrodynamic signals in order to better investigate the relationship of each component with the far field. Furthermore, investigation of different frequencies and modes may help shed some light regarding the near-field dynamics of the jet. 


\section{References}

[1] Jordan, P., and Gervais, Y., 2008, "Subsonic Jet Aeroacoustics: Associating Experiment, Modelling and Simulation," Experiments in Fluids, 44(1), pp. 1-21.

[2] Samimy, M., Zaman, K. B. M. Q., and Reeder, M. F., 1993, "Effect of Tabs on the Flow and Noise Field of an Axisymmetric Jet," AIAA Journal, 31(4), pp. 609-619.

[3] Zaman, K. B. M. Q., Reeder, M. F., and Samimy, M., 1994, "Control of an axisymmetric jet using vortex generators," Phys. Fluids, 6(2), pp. 778-793.

[4] Kim, J.-H., and Samimy, M., 1999, "Mixing enhancement via nozzle trailing edge modifications in a high speed rectangular jet," Phys. Fluids, 11(9), pp. 2731-2742.

[5] Callender, B., Gutmark, E., and Martens, S., 2004, "Far-Field Acoustic Investigation into Chevron Nozzle Mechanisms and Trends," AIAA Journal, 43(1), pp. 87-95.

[6] Viswanathan, K., 2005, "Nozzle Shaping for Reduction of Jet Noise from Single Jets," AIAA Journal, 43(5), pp. 1008-1022.

[7] Crow, S. C., and Champagne, F. H., 1971, "Orderly structure in jet turbulence," Journal of Fluid Mechanics, 48(3), pp. 547-591.

[8] Kibens, V., 1980, "Discrete Noise Spectrum Generated by an Acoustically Excited Jet," AIAA Journal, 18(4), pp. 434-441.

[9] Zaman, K. B. M. Q., and Hussain, A. K. M. F., 1980, "Vortex pairing in a circular jet under controlled excitation. Part 1. General jet response," J. Fluid Mech., 101(3), pp. 449-491.

[10] Zaman, K. B. M. Q., and Hussain, A. K. M. F., 1981, "Turbulence suppression in free shear flows by controlled excitation," J. Fluid Mech., 103, pp. 133-159.

[11] Gutmark, E., and Ho, C.-M., 1983, "Preferred modes and the spreading rates of jets," Phys. Fluids, 26(10), pp. 2932-2938.

[12] Ho, C.-M., and Huerre, P., 1984, "Perturbed free shear layers," Annu. Rev. Fluid Mech., 16, pp. 365-424.

[13] Cohen, J., and Wygnanski, I., 1987, "The evolution of instabilities in the axisymmetric jet. Part 1. The linear growth of disturbances near the nozzle," J. Fluid Mech., 176, pp. 191-219.

[14] Jendoubi, S., and Strykowski, P. J., 1994, "Absolute and Convective Instability of Axisymmetric Jets with External Flow," Phys. Fluids, 6(9), pp. 3000-3009.

[15] Samimy, M., Kim, J. H., Kastner, J., Adamovich, I., and Utkin, Y., 2007, "Active Control of a Mach 0.9 Jet for Noise Mitigation Using Plasma Actuators," AIAA J., 45(4), pp. 890-901.

[16] Samimy, M., Kim, J.-H., Kastner, J., Adamovich, I., and Utkin, Y., 2007, "Active control of high-speed and high-Reynolds-number jets using plasma actuators," J. Fluid Mech., 578, pp. 305-330.

[17] Jubelin, B., 1980, "New Experimental Studies on Jet Noise Amplification," AIAA 6th Aeroacoustics ConferenceHartford, Conn., June 4-6, p. 8. 
[18] Lu, H. Y., 1983, "Effect of Excitation on Coaxial Jet Noise," AIAA Journal, 21(2), pp. 214-220.

[19] Ahuja, K. K., and Blakney, D. F., 1985, "Tone Excited Jets, Part IV: Acoustic Measurements," J. Sound \& Vib., 102(1), pp. 93-117.

[20] Samimy, M., Kim, J.-H., Kearney-Fischer, M., and Sinha, A., 2010, "Acoustic and Flow Fields of an Excited High Reynolds Number Axisymmetric Supersonic Jet," Journal of Fluid Mechanics, 656, pp. 507-529.

[21] Kearney-Fischer, M., Kim, J.-H., and Samimy, M., 2009, "Control of a high Reynolds number Mach 0.9 heated jet using plasma actuators," Physics of Fluids, 21, p. 095101.

[22] Kim, J.-H., Kastner, J., and Samimy, M., 2009, "Active Control of a High Reynolds Number Mach 0.9 Axisymmetric Jet," AIAA Journal, 47(1), pp. 116-128.

[23] Kearney-Fischer, M., Kim, J.-H., and Samimy, M., 2011, "A Study of Mach Wave Radiation Using Active Control," J. Fluid Mech., 681, pp. 261-292.

[24] Kearney-Fischer, M., Kim, J.-H., and Samimy, M., 2011, "Noise Control of a High Reynolds Number High Speed Heated Jet Using Plasma Actuators," Int. J. Aeroacoustics, 10(5-6), pp. $635-658$.

[25] Sinha, A., Kim, K., Kim, J., Serrani, A., and Samimy, M., 2010, "Extremizing Feedback Control of a High-Speed and High Reynolds Number Jet," AIAA Journal, 48(2), pp. 387-399.

[26] Suzuki, T., and Colonius, T., 2006, "Instability waves in a subsonic round jet detected using a near-field phased microphone array," Journal of Fluid Mechanics, 565, pp. 197-226.

[27] Tinney, C. E., and Jordan, P., 2008, "The near pressure field of co-axial subsonic jets," Journal of Fluid Mechanics, 611, pp. 175-204.

[28] Sinha, A., Alkandry, H., Kearney-Fischer, M., Samimy, M., and Colonius, T., 2012, "The Impulse Response of a High-Speed Jet Forced with Localized Arc Filament Plasma Actuators," Phys. Fluids, 24, pp. 24-44.

[29] Arndt, R. E. A., Long, D. F., and Glauser, M. N., 1997, "The proper orthogonal decomposition of pressure fluctuations surrounding a turbulent jet," Journal of Fluid Mechanics, 340, pp. 1-33.

[30] Coiffet, F., Jordan, P., Oelville, J., Gervais, Y., and Ricaud, F., 2006, "Coherent structures in subsonic jets: a quasi-irrotational source mechanism?," International Journal of Aeroacoustics, 5(1), pp. 67 - 89.

[31] Schlichting, H., 1968, Boundary Layer Theory, McGraw-Hill, New York

[32] Pope, S. B., 2000, Turbulent Flows, Cambridge University Press, Cambridge UK.

[33] Tam, C. K. W., Viswanathan, K., Ahuja, K., and Panda, J., 2008, "The Source of Jet Noise: Experimental Evidence," J. Fluid Mech., 615, pp. 253-292.

[34] Brown, G. L., and Roshko, A., 1974, "On density effects and large structure in turbulent mixing layers," J. Fluid Mech., 64(4), pp. 775-816.

[35] Ffowcs Williams, J. E., 1963, "The Noise From Turbulence Convected At High Speed," Philosophical Transactions of the Royal Society of London: Series A, 255(1061), pp. 469-503. 
[36] Lilley, G. M., 1991, "Jet Noise Classical Theory and Experiments," Aeroacoustics of Flight Vehicles: Theory and Practice, 1, pp. 211-289.

[37] Tam, C. K. W., Chen, P., and Seiner, J. M., 1991, "Relationship Between Instability Waves and Noise of High-Speed Jets," AIAA Journal, 30(7), pp. 1747-1752.

[38] Hileman, J., and Samimy, M., 2001, "Turbulence Structures and the Acoustic Far Field of a Mach 1.3 Jet," AIAA Journal, 39(9), pp. 1716-1727.

[39] Tam, C. K. W., and Chen, P., 1994, "Turbulent Mixing Noise From Supersonic Jets," AIAA Journal, 32(9), pp. 1774-1780.

[40] Tam, C. K. W., 1995, "Supersonic Jet Noise," Annual Review of Fluid Mechanics, 27, pp. 17-43.

[41] Tam, C. K. W., Golebiowski, M., and Seiner, J. M., 1996, "On the Two Components of Turbulent Mixing Noise from Supersonic Jets," AIAA/CEAS 2nd Aeroacoustics ConferenceState College, PA, May 6-8.

[42] Dahl, M. D., and Papamoschou, D., 2000, "Analytical Predictions and Measurements of the Noise Radiated from Supersonic Coaxial Jets," AIAA Journal, 38(4), pp. 584-591.

[43] Viswanathan, K., 2002, "Analysis of the Two Similarity Components of Turbulent Mixing Noise," AIAA Journal, 40(9), pp. 1735-1744.

[44] Tam, C. K. W., 1991, "Jet Noise Generated by Large-Scale Coherent Motion.," Aeroacoustics of Flight Vehicles: Theory and Practice., Vol. 1, pp. 311-390.

[45] Schaffar, M., 1979, "Direct Measurements of the Correlation Between Axial In-Jet Velocity Fluctuations and Far Field Noise Near the Axis of a Cold Jet," Journal of Sound and Vibration, 64(1), pp. 73-83.

[46] Morrison, G. L., and McLaughlin, D. K., 1979, "Noise Generation by Instabilities in Low Reynolds Number Supersonic Jets," J. Sound \& Vib., 65(2), pp. 177-191.

[47] Narayanan, S., Barber, T. J., and Polak, D. R., 2002, "High Subsonic Jet Experiments: Turbulence and Noise Generation Studies," AIAA Journal, 40(3), pp. 430-437.

[48] Hileman, J., Thurow, B., and Samimy, S., 2004, "Development and evaluation of a 3-D microphone array to locate individual acoustic sources in a high-speed jet," J. Sound \& Vib., 276(3-5), pp. 649-669.

[49] Lee, H. K., and Ribner, H. S., 1972, "Direct Correlation of Noise and Flow of a Jet," Journal of the Acoustical Society of America, 52(5), pp. 1280-1290.

[50] Michalke, A., 1965, "On spatially growing disturbances in an inviscid shear layer," J. Fluid Mech., 23(3), pp. 521-544.

[51] Samimy, M., Kim, J. H., Kastner, J., Adamovich, I., and Utkin, Y., 2007, "Active Control of High-Speed and High-Reynolds-Number Jets Using Plasma Actuators," Journal of Fluid Mechanics, 578(1), pp. 305-330.

[52] Samimy, M., Kim, J.-H., Kastner, J., Adamovich, I., and Utkin, Y., 2007, "Active Control of a Mach 0.9 Jet for Noise Mitigation Using Plasma Actuators," AIAA Journal, 45(4), pp. 890-901.

[53] Henderson, B., Bridges, J., and Wernet, M. P., 2005, "An experimental study of the oscillatory flow structure of tone-producing supersonic impinging jets," J. Fluid Mech., 542, pp. 115-137. 
[54] Kastner, J., and Samimy, M., 2002, "Development and Characterization of Hartmann Tube Fluidic Actuators for High-Speed Flow Control," AIAA Journal, 40(10), pp. 1926-1934.

[55] Butler, G. W., and Calkins, F. T., 2003, "Initial attempts to suppress jet noise using piezoelectric actuators," 9th AIAA/CEAS, 2003-3192, p. 15.

[56] Samimy, M., Kim, J.-H., Kastner, J., Adamovich, I., and Utkin, Y., 2007, "Active Control of High-Speed and High-Reynolds-Number Jets Using Plasma Actuators," J. Fluid Mech., 578(1), pp. 305-330.

[57] Howes, W., 1960, "Distribution of time-averaged pressure fluctuations along the boundary of a round subsonic jet," NASA Technical Note D-468.

[58] Mollo-Christensen, E., 1963, "Measurements of near field pressure of subsonic jets," NATO A.G.A.R.D. Report 449.

[59] Hahn, C., 2011, "Design and Validation of the New Jet Facility and Anechoic Chamber," M.S., The Ohio State University, Columbus.

[60] Hahn, C., Kearney-Fischer, M., and Samimy, M., 2011, "On factors influencing arc filament plasma actuator performance in control of high speed jets," Exp. Fluids, $37(5)$.

[61] Samimy, M., Adamovich, I., Webb, B., Kastner, J., Hileman, J., Keshav, S., and Palm, P., 2004, "Development and characterization of plasma actuators for highspeed jet control," Experiments in Fluids, 37(4), pp. 577-588.

[62] Utkin, Y. G., Keshav, S., Kim, J.-H., Kastner, J., Adamovich, I. V., and Samimy, M., 2007, "Development and Use of Localized Arc Filament Plasma Actuators for High-Speed Flow Control," Journal of Physics D: Applied Physics, 40(3), pp. 685694.

[63] Kim, J., Nishihara, M., Adamovich, I., Samimy, M., Gorbatov, S. V., and Pliavaka, F. V., 2010, "Development of Localized Arc Filament RF Plasma Actuators for High-Speed and High Reynolds Number Flow Control," Exp. Fluids, 49, pp. 497511.

[64] Hahn, C., Kearney-Fischer, M., and Samimy, M., 2011, "Effects of Ring Groove and Duty Cycle on Plasma Actuator Performance in High Speed Jets," 49th AIAA Aerospace Sciences Meeting.

[65] Harper-Bourne, M., 2004, "On Modelling the Hydrodynamic Field of High-Speed Jets," AIAA/CEAS 10th Aeroacoustics Conference.

[66] Picard, C., and Delville, J., 2000, "Pressure velocity coupling in a subsonic round jet," International Journal of Heat and Fluid Flow, 21(3), pp. 359-364.

[67] Ukeiley, L. S., and Ponton, M. K., 2004, "On the near field pressure of a transonic axisymmetric jet," International Journal of Aeroacoustics, 3(1), pp. 43-66.

[68] Tam, C. K. W., Viswanathan, K., Ahuja, K. K., and Panda, J., 2008, "The sources of jet noise: experimental evidence," Journal of Fluid Mechanics, 615, pp. 253-292.

[69] Hall, J. W., Pinier, J., Hall, A. e., and Glauser, M., 2006, "Two-point correlations of the near and far-field pressure in a transonic jet," ASME 2006 Joint U.S. European Fluids Engineering Summer Meeting. 